TRANSACTIONS OF THE

AMERICAN MATHEMATICAL SOCIETY

Volume 365, Number 1, January 2013, Pages 409-458

S 0002-9947(2012)05616-9

Article electronically published on August 8, 2012

\title{
ON THE STOCHASTIC HEAT EQUATION WITH SPATIALLY-COLORED RANDOM FORCING
}

\author{
MOHAMMUD FOONDUN AND DAVAR KHOSHNEVISAN
}

ABstract. We consider the stochastic heat equation of the following form:

$$
\frac{\partial}{\partial t} u_{t}(x)=\left(\mathcal{L} u_{t}\right)(x)+b\left(u_{t}(x)\right)+\sigma\left(u_{t}(x)\right) \dot{F}_{t}(x) \text { for } t>0, x \in \mathbf{R}^{d},
$$

where $\mathcal{L}$ is the generator of a Lévy process and $\dot{F}$ is a spatially-colored, temporally white, Gaussian noise. We will be concerned mainly with the long-term behavior of the mild solution to this stochastic PDE.

For the most part, we work under the assumptions that the initial data $u_{0}$ is a bounded and measurable function and $\sigma$ is nonconstant and Lipschitz continuous. In this case, we find conditions under which the preceding stochastic PDE admits a unique solution which is also weakly intermittent. In addition, we study the same equation in the case where $\mathcal{L} u$ is replaced by its massive/dispersive analogue $\mathcal{L} u-\lambda u$, where $\lambda \in \mathbf{R}$. We also accurately describe the effect of the parameter $\lambda$ on the intermittence of the solution in the case where $\sigma(u)$ is proportional to $u$ [the "parabolic Anderson model"].

We also look at the linearized version of our stochastic PDE, that is, the case where $\sigma$ is identically equal to one [any other constant also works]. In this case, we study not only the existence and uniqueness of a solution, but also the regularity of the solution when it exists and is unique.

\section{INTRODUCTION AND STATEMENTS OF MAIN RESULTS}

The principle aim of this paper is to describe the asymptotic large-time behavior of the mild solution $u:=\left\{u_{t}(x)\right\}_{t \geq 0, x \in \mathbf{R}^{d}}$ to the stochastic heat equation,

$$
\frac{\partial}{\partial t} u_{t}(x)=\left(\mathcal{L} u_{t}\right)(x)+b\left(u_{t}(x)\right)+\sigma\left(u_{t}(x)\right) \dot{F}_{t}(x),
$$

where $t>0$ and $x \in \mathbf{R}^{d}$, where the preceding stochastic PDE can be understood in the sense of Walsh [92 and Dalang [30. We have used, and will be using, the standard notation of probability theory throughout: Namely, $g_{t}$ denotes the evaluation of a random or nonrandom function $g$ at time $t$, and never the time derivative of $g$.

We consider only the case where the initial data $u_{0}$ is a nonrandom, as well as bounded and measurable, function. The functions $\sigma, b: \mathbf{R} \rightarrow \mathbf{R}$ are nonrandom and Lipschitz continuous. Also, we let $\mathcal{L}$ be the $L^{2}$-generator of a $d$-dimensional Lévy process $X:=\left\{X_{t}\right\}_{t \geq 0}$, and assume that $X$ has transition functions.

Received by the editors April 18, 2011.

2010 Mathematics Subject Classification. Primary 60H15; Secondary 35R60.

Key words and phrases. The stochastic heat equation, spatially-colored homogeneous noise, Lévy processes.

This research was supported in part by grants from the National Science Foundation. 
As regards the forcing term $\dot{F}$ in (1.1), we assume that $\dot{F}$ is a generalized Gaussian random field [50, Chapter 2, §2.4] whose covariance kernel is $\delta_{0}(s-t) f(x-y)$, where the "correlation function" $f$ is a nonnegative definite, symmetric, and nonnegative function that is not identically zero. The symbol " $f$ " is reserved for this correlation function here and throughout. We never refer to any other function as $f$. Alternatively, one can use

$$
\dot{F}_{t}(x):=\frac{\partial^{d+1}}{\partial t \partial x_{1} \cdots \partial x_{d}} F(t, x)
$$

in the sense of generalized random fields, where $F$ is a centered generalized Gaussian random field with covariance kernel

$$
\begin{aligned}
\operatorname{Cov}\left(\int \phi \mathrm{d} F, \int \zeta \mathrm{d} F\right) & =\int_{0}^{\infty} \mathrm{d} s \int_{\mathbf{R}^{d}} \mathrm{~d} x \int_{\mathbf{R}^{d}} \mathrm{~d} y \phi_{s}(x) \zeta_{s}(y) f(x-y) \\
& =\int_{0}^{\infty}\left(\phi_{s}, f * \zeta_{s}\right)_{L^{2}\left(\mathbf{R}^{d}\right)} \mathrm{d} s,
\end{aligned}
$$

where $\int \phi \mathrm{d} F$ and $\int \zeta \mathrm{d} F$ are Wiener integrals of $\mathbf{R}_{+} \times \mathbf{R}^{d} \ni(s, x) \mapsto \phi_{s}(x)$ and $\mathbf{R}_{+} \times \mathbf{R}^{d} \ni(s, x) \mapsto \zeta_{s}(x)$ with respect to $F$, and $\phi$ and $\zeta$ are nonnegative measurable functions for which the right-most multiple integral in (1.3) converges absolutely. We mention that the generalized Gaussian random field $(t, \phi) \mapsto \int \phi \mathrm{d} F$ continues to make sense when the correlation $f$ is a measure, or more generally a Schwartz distribution, provided that we interpret the covariance functional in (1.3) as the final integral that appears in that display. However, we will not consider such generalizations, primarily because we do not know how to prove Theorems 1.8 and 1.11 below except when $f$ has reasonable function structure.

According to the Bochner-Schwartz theorem [50, Theorem 3, p. 157]: (a) the Fourier transform $\hat{f}$ of $f$ is a [nonnegative Borel] tempered measure on $\mathbf{R}^{d}$; (b) conversely, every tempered measure $\hat{f}$ on $\mathbf{R}^{d}$ is the Fourier transform of one such correlation function $f$. The measure $\hat{f}$ is known as the "spectral measure" of the noise $F$. Throughout, we assume without further mention that $F$ "has a spectral density". That is,

$$
\hat{f} \text { is a measurable function. }
$$

This implies that $\hat{f}$ is locally integrable on $\mathbf{R}^{d}$ as well. Strictly speaking, these conditions are not always needed in our work, but we assume them for the sake of simplicity.

By enlarging the underlying probability space, if need be, we introduce an independent copy $X^{*}:=\left\{X_{t}^{*}\right\}_{t \geq 0}$ of the dual process $-X$. We can then use $X^{*}$ to define a symmetric Lévy process $\bar{X}:=\left\{\bar{X}_{t}\right\}_{t \geq 0}$ on $\mathbf{R}^{d}$ via the assignment

$$
\bar{X}_{t}:=X_{t}+X_{t}^{*} \quad \text { for all } t \geq 0 .
$$

Motivated by the works of Kardar, Parisi, and Zhang [60] and Kardar [59], we may refer to $\bar{X}$ as the replica Lévy process corresponding to $X$ and will therefore call the resolvent $\left\{\bar{R}_{\alpha}\right\}_{\alpha>0}$ of $\bar{X}$ the replica resolvent. These quantities are defined in more detail in 92

We will consider the condition that the correlation function $f$ has finite $\alpha$ potential at zero for all $\alpha>0$. That is, we consider the following:

Condition 1.1. $\left(\bar{R}_{\alpha} f\right)(0)<\infty$ for all $\alpha>0$. 
It will turn out that Condition 1.1 allows one to apply the theory of Dalang [30] to the present setting and obtain an existence and uniqueness result for the stochastic heat equation (1.1). Here we show that the existence and uniqueness of a solution is closely linked to the large-time behavior of the solution itself [via a priori estimates]. As mentioned above, our solution is of mild form; it can be written as

$$
\begin{aligned}
u_{t}(x)=\left(P_{t} u_{0}\right)(x)+\int_{0}^{t} \mathrm{~d} s \int_{\mathbf{R}^{d}} \mathrm{~d} y p_{t-s}(y-x) b\left(u_{s}(y)\right) \\
\quad+\int_{0}^{t} \int_{\mathbf{R}^{d}} p_{t-s}(y-x) \sigma\left(u_{s}(y)\right) F(\mathrm{~d} s \mathrm{~d} y),
\end{aligned}
$$

where $\left\{P_{t}\right\}_{t>0}$ is the semigroup of $X$, defined in $\S 2$, and $p_{t}(\cdot)$ is the transition function of $X$. Next we describe these results in greater detail. But first, let us define two important quantities: The first denotes the upper $L^{p}(\mathrm{P})$-Liapounov exponent of the solution $u:=\left\{u_{t}(x)\right\}_{t>0, x \in \mathbf{R}^{d}}$ to (1.1) at the spatial point $x \in \mathbf{R}^{d}$ :

$$
\bar{\gamma}_{x}(p):=\limsup _{t \rightarrow \infty} \frac{1}{t} \ln \mathrm{E}\left(\left|u_{t}(x)\right|^{p}\right),
$$

and the second the upper maximum $L^{p}(\mathrm{P})$-Liapounov exponent of $u$ :

$$
\bar{\gamma}_{*}(p):=\limsup _{t \rightarrow \infty} \frac{1}{t} \sup _{x \in \mathbf{R}^{d}} \ln \mathrm{E}\left(\left|u_{t}(x)\right|^{p}\right) .
$$

The above two quantities are variants of Liapounov exponents.

Define for all $\beta \geq 0$,

$$
\Upsilon(\beta):=\frac{1}{(2 \pi)^{d}} \int \frac{\hat{f}(\xi)}{\beta+2 \operatorname{Re} \Psi(\xi)} \mathrm{d} \xi,
$$

where $\Psi$ is the characteristic exponent of the Lévy process $X$. Dalang [30] has established a very general result which guarantees the existence and uniqueness of solutions to large families of SPDEs. If we apply Dalang's result to the present parabolic problem (1.1), then we find the following: The condition

$$
\Upsilon(1)<\infty
$$

ensures the existence and uniqueness of a [mild] solution to (1.1). [The main result of [30] is stated for the case where $u_{0}$ is a constant, but its derivation applies equally well to the present case where $u_{0}$ is bounded and measurable.]

In addition, Dalang 30 has shown that (1.9) cannot be improved upon: (1.9) is necessary and sufficient for the existence and uniqueness of a solution in the case that (1.1) is a linear SPDE $[\sigma \equiv 1, b \equiv 0]$. There are also many closely related results including analyses of hyperbolic equations [19, 25, 31, 35, 36, 37, 83.

We are now ready to state the first main contribution of our paper. Our first result implies among many other things that Dalang's condition (1.9) is generically equivalent to the potential-theoretic Condition 1.1

Theorem 1.2 (A maximum principle). For all $\beta>0$,

$$
\left(\bar{R}_{\beta} f\right)(0)=\sup _{x \in \mathbf{R}^{d}}\left(\bar{R}_{\beta} f\right)(x)=\Upsilon(\beta) .
$$

Thus, Condition 1.1 holds if and only if (1.9) holds. Furthermore, if Condition 1.1 $[$ and/or (1.9)] holds and $f$ is lower semicontinuous, then for all $\beta>0$ there exists $\pi_{\beta} \in C_{0}\left(\mathbf{R}^{d}\right)$ such that $\bar{R}_{\beta} f=\pi_{\beta}$ almost everywhere. 
$\left\{\bar{R}_{\beta}\right\}_{\beta>0}$ is the resolvent of a self-dual semigroup - namely the semigroup of the Lévy process $\bar{X}$-and, because of (2.8) below, the theory of [14] applies and tells us that $\left(\bar{R}_{\beta} h\right)(x)$ is maximized at $x=0$ for all $h \in L^{1}\left(\mathbf{R}^{d}\right)$. Theorem 1.2 tells us that the integrability condition can be replaced by positive definiteness without changing the end result. Our proof turns out to be Fourier analytic and quite different from the balayage arguments of potential theory 14 .

Theorem 1.2 implies that, under Condition 1.1. we may apply Dalang's theory, and therefore (1.1) has an a.s.-unique solution which can be written in mild form. Our next result provides information about the upper maximum $L^{p}(\mathrm{P})$-Liapounov exponent of that solution.

Theorem 1.3. Assume Condition 1.1 and suppose $u_{0}: \mathbf{R}^{d} \rightarrow \mathbf{R}$ is bounded and measurable. Then

$$
\bar{\gamma}_{*}(p) \leq \inf \{\beta>0: Q(p, \beta)<1\} \quad \text { for all integers } p \geq 2,
$$

where

$$
Q(p, \beta):=\frac{p \operatorname{Lip}_{b}}{\beta}+z_{p} \operatorname{Lip}_{\sigma} \sqrt{\left(\bar{R}_{2 \beta / p} f\right)(0)},
$$

and $z_{p}$ denotes the largest positive zero of the Hermite polynomial $\mathrm{He}_{p}$.

The proof of Theorem 1.3 is a potential-theoretic abstraction of our Fourieranalytic arguments in [45. Theorem 2.1] that were devised to study the heat equation (1.1) driven by space-time white noise [in place of $\dot{F}$ ]. The present potentialtheoretic methods can be generalized further to include operators $\mathcal{L}$ that are generators of much more general Markov processes, but we will not pursue those extensions here.

Let us make two remarks before we continue with our presentation of the main results of this paper.

Remark 1.4. It is possible to deduce from Condition 1.1 and the monotone convergence theorem that $\lim _{\alpha \rightarrow \infty}\left(\bar{R}_{\alpha} f\right)(0)=0$. This, in turn, implies that $Q(p, \beta) \rightarrow 0$ as $\beta \rightarrow \infty$. Consequently, Theorem 1.3 implies, among other things, that $\bar{\gamma}_{*}(p)<\infty$ for all $p \in(0, \infty)$.

Remark 1.5 (Borrowed from [4, Remark 2.2]). It might help to recall that

$$
\operatorname{He}_{k}(x)=2^{-k / 2} H_{k}(x / \sqrt{2}) \quad \text { for all integers } k \geq 0 \text { and } x \in \mathbf{R},
$$

where $\left\{H_{k}\right\}_{k=0}^{\infty}$ is defined uniquely via the following:

$$
\mathrm{e}^{-2 x t-t^{2}}=\sum_{k=0}^{\infty} \frac{t^{k}}{k !} H_{k}(x) \quad(t>0, x \in \mathbf{R}) .
$$

It is not hard to verify that

$$
z_{2}=1 \quad \text { and } \quad z_{4}=\sqrt{3+\sqrt{6}} \approx 2.334 .
$$

This is valid simply because $\mathrm{He}_{2}(x)=x^{2}-1$ and $\mathrm{He}_{4}(x)=x^{4}-6 x^{2}+3$. In addition,

$$
\lim _{p \rightarrow \infty}\left(\frac{z_{p}}{\sqrt{p}}\right)=\sup _{p \geq 1}\left(\frac{z_{p}}{\sqrt{p}}\right)=2
$$

see Carlen and Kree [16, Appendix]. 
In order to pave the way for the second main result of this paper, we recall the following [45]:

Definition 1.6. Suppose (1.1) has an a.s.-unique solution $u:=\left\{u_{t}(x)\right\}_{t>0, x \in \mathbf{R}^{d}}$. We say that $u$ is weakly intermittent if $\bar{\gamma}_{*}(p)$ is finite for all $p \in[2, \infty)$ and $\inf _{x \in \mathbf{R}^{d}} \bar{\gamma}_{x}(2)>0$.

The same reasoning that was employed in [45] can be used to deduce that if the solution to (1.1) is nonnegative for all $t>0$, then weak intermittence implies the much better-known property of intermittency [19, 77, 96]; that is, the property that $p \mapsto \bar{\gamma}_{x}(p) / p$ is strictly increasing on $[2, \infty)$ for all $x \in \mathbf{R}^{d}$. There is a large literature which shows that, under further mild hypotheses on $\mathcal{L}$ and/or $f$, if $u_{0}$ is nonnegative, then the solution to (1.1) is nonnegative at all times [1, 19, 40, 53, 63, 69, 71, 70, 78, 81, 87, 86. In connection to matters of positivity and regularity, we also mention the closely related paper [39, where (1.1) is considered with $\sigma(u)=$ const $\cdot \sqrt{u}$. Positivity of the solution is shown to follow from manyparticle approximations to the underlying SPDE.

Thus, we can draw the conclusion that, in all such cases, weak intermittence actually implies intermittency.

A quick calculation, using only Hölder's inequality, shows that $p \mapsto \bar{\gamma}_{x}(p) / p$ is always nondecreasing on $[2, \infty)$. However, the mentioned strict monotonicity does not always hold. When it does hold, then it has some physical significance [77, 96].

Our next main goal is to find nontrivial conditions that guarantee the weak intermittence of the solution to (1.1). In light of Theorem 1.3, we aim to derive a positive lower bound on $\inf _{x \in \mathbf{R}^{d}} \bar{\gamma}_{x}(2)$. Unfortunately, it is quite hard to do this at the level of generality of the conditions of Theorem [1.3, and we are able to establish weak intermittency only when certain technical conditions hold. In order to describe those technical conditions, let $\hat{g}$ denote the Fourier transform of a locally integrable function $g$, and consider the following:

Condition 1.7. Suppose:

(1) $\hat{f}(\xi)$ depends on $\xi \in \mathbf{R}^{d}$ only through $\left|\xi_{1}\right|, \ldots,\left|\xi_{d}\right|$;

(2) $\left|\xi_{j}\right| \mapsto \hat{f}(\xi)$ is nonincreasing for every $j=1, \ldots, d$; and

(3) $\operatorname{Re} \Psi(\xi)$ depends on $\xi \in \mathbf{R}^{d}$ only through $\left|\xi_{1}\right|, \ldots,\left|\xi_{d}\right|$.

These are relatively mild provisions on the spectral density $\hat{f}$ and the process $\bar{X}$. Our conditions on the spectral density can be applied to all of the examples that we would like to cover. It is possible to show that they include the following choices for $f$ :

(i) Ornstein-Uhlenbeck-type kernels. $f(x)=\exp \left(-\|x\|^{\alpha}\right)$ for $\alpha \in(0,2], \hat{f}(\xi)=$ $(2 \pi)^{-d} \int_{\mathbf{R}^{d}} \exp \left(-i \xi \cdot x-\|x\|^{\alpha}\right) \mathrm{d} x$

(ii) Poisson kernels. $f(x)=\left(\|x\|^{2}+1\right)^{-(d+1) / 2}, \hat{f}(\xi)=c_{1} \exp \left(-c_{2}\|\xi\|\right)$ for some $c_{1}, c_{2} \in(0, \infty)$

(iii) Cauchy kernels. $f(x)=\prod_{j=1}^{d}\left(1+x_{j}^{2}\right)^{-1}, \hat{f}(\xi)=c_{1} \exp \left\{c_{2} \sum_{j=1}^{d}\left|\xi_{j}\right|\right\}$ for some $c_{1}, c_{2} \in(0, \infty)$; and

(iv) Riesz kernels. $f(x)=\|x\|^{-\alpha}, \hat{f}(\xi)=c\|\xi\|^{-d+\alpha}$ for $\alpha \in(0, d)$ and a suitable $c \in(0, \infty)$.

Moreover, one can construct a great number of other permissible examples as well.

Having introduced Condition 1.7. we can now present the third main result of this paper. 
Theorem 1.8. Suppose $b \equiv 0$ and Conditions 1.1 and 1.7 hold. Suppose, in addition, that $\eta:=\inf _{x \in \mathbf{R}^{d}} u_{0}(x)>0$ and there exists $\mathrm{L}_{\sigma} \in(0, \infty)$ such that $\sigma(z) \geq \mathrm{L}_{\sigma}|z|$ for all $z \in \mathbf{R}$. Then

$$
\inf _{x \in \mathbf{R}^{d}} \bar{\gamma}_{x}(2) \geq \sup \left\{\beta>0:\left(\bar{R}_{\beta} f\right)(0) \geq \frac{2^{d-1}}{\mathrm{~L}_{\sigma}^{2}}\right\},
$$

where $\sup \varnothing:=0$.

Consider, for an example, the case where $\left(\bar{R}_{0} f\right)(0)=\infty$, whence $\left(R_{\beta} f\right)(0) \uparrow \infty$ as $\beta \downarrow 0$. Consequently, $\left(\bar{R}_{\beta} f\right)(0) \geq 2^{d-1} / \mathrm{L}_{\sigma}^{2}$ for all $\beta>0$ sufficiently small. Hence, whenever $\left(\bar{R}_{0} f\right)(0)=\infty$, the hypotheses of Theorem 1.8 guarantee weak intermittence of the solution to (1.1) without further restrictions. The condition $\left(\bar{R}_{0} f\right)(0)=\infty$ is basically stating that $f$ is a recurrent function for the Lévy process $\bar{X}$; for a precise statement see Proposition 3.13 below.

Remark 1.9. (1) In the case where $\dot{F}$ is space-time white noise, the condition " $\sigma(z) \geq \mathrm{L}_{\sigma}|z|$ " can be replaced with the slightly better condition " $|\sigma(z)| \geq$ $\mathrm{L}_{\sigma}|z| "$ [45]. But the methods employed here are different in a few [quite essential] spots from the techniques of [45].

(2) We will see later on that, when $d=1$, the lower bound (1.17) and the upper bound (1.11) can sometimes match. However, the two bounds can never agree when $d \geq 2$. This phenomenon is due to the fact that level sets of $\beta \mapsto\left(\bar{R}_{\beta} f\right)(0)$ cannot exactly describe the growth of $u$. The correct gauge appears to be a much more complicated function, except in the cases where $\dot{F}$ is space-time white noise and when $d=1$. Compare this with [45] for results on the case where $\dot{F}$ denotes space-time white noise.

We are aware of a few variants of Theorem 1.8, but the next one is perhaps the most striking since it assumes only that the nonlinearity term $\sigma$ is asymptotically sublinear. Thus, the local behavior of $\sigma$ is shown not to have an effect on weak intermittence, provided that the initial data $u_{0}$ is sufficiently large. A significant drawback of this result is that its proof does not provide any information about how large "sufficiently large" should be. We introduce the following condition.

Condition 1.10. $\left(\bar{R}_{0} f\right)(0)=\infty$.

The following result is the mentioned variant of Theorem 1.8 and has a similar flavor to [45, Theorem 2.10], though its method of proof differs significantly, in key spots, from the arguments of [45].

Theorem 1.11. Suppose $b \equiv 0$ and Conditions 1.1, 1.7, and 1.10 hold. Suppose, in addition, that $\sigma \geq 0$ pointwise, and $q:=\liminf _{|z| \rightarrow \infty} \sigma(z) /|z|>0$. If $u_{0}(x)>0$ and $\mathrm{P}\left\{u_{t}(x)>0\right\}=1$ for all $t>0$ and $x \in \mathbf{R}^{d}$, then $\bar{\gamma}_{x}(2)>0$ for every $x \in \mathbf{R}^{d}$ provided that $\eta:=\inf _{x \in \mathbf{R}^{d}} u_{0}(x)$ is sufficiently large.

Theorems 1.3, 1.8, and 1.11 describe our main contributions to the analysis of the stochastic heat equation (1.1) in the case where $\sigma$ is not a constant and that $u_{0}$ is a bounded and measurable function. We also study the linearization of (1.1); this is the case when $\sigma$ is identically equal to one. The general theory of Gaussian processes readily resolves all existence-and-uniqueness issues; we use that theory to study continuity properties of the solution. Moreover, we produce a class of interesting examples which we briefly describe next. 
Consider the linear stochastic heat equation

$$
\frac{\partial}{\partial t} u_{t}(x)=\left(\Delta u_{t}\right)(x)+\dot{F}_{t}(x) \quad\left(t>0, x \in \mathbf{R}^{3}\right)
$$

where $u_{0} \equiv 0$ and the Laplacian acts on the $x$ variable only. Then, we construct families of noises $\dot{F}$ which ensure that (1.18) has a solution $u:=\left\{u_{t}(x)\right\}_{t \geq 0, x \in \mathbf{R}^{3}}$ that is a square-integrable random field. But that random field is discontinuous densely with probability one. In fact, outside of a single null set [of realizations of the process $u$,

$$
\sup _{(t, x) \in V} u_{t}(x)=-\inf _{(t, x) \in V} u_{t}(x)=\infty
$$

for all open balls $V \subset \mathbf{R}_{+} \times \mathbf{R}^{d}$ with rational centers and radii! We know of only a few examples of SPDEs with well-defined random-field solutions that have densely unbounded oscillations [34, 33, 32, 79, 46]. The preceding (1.19) yields a quite simple example of an otherwise physically-natural stochastic PDE [the operator is the Laplacian in $\mathbf{R}^{3}$, and the noise is white in time] which has a very badly behaved solution.

This paper was influenced greatly by the theoretical physics literature on the "parabolic Anderson model" (see, for example, [60, 75, 96] and [64, §5]), as well as the mathematical physics literature on the very same topic [6, 5, 4, 8, 7, 18, 17, 19, 20, 24, 23, 22, 27, 26, 29, 28, 44, 48, 49, 91, 62, 67, 77, 93. In a nutshell, the parabolic Anderson model is equation (1.1), where $\sigma(u)$ is proportional to $u$. There are many good reasons why that equation has been studied intensively 19 , Introduction]. Two such reasons are that the parabolic Anderson model is exactly solvable in the two cases where $u_{0} \equiv$ constant and $u_{0}=\delta_{0}$ and that it is related to the stochastic Burgers equation as well as the KPZ equation of statistical mechanics.

Perhaps not surprisingly, the results of our Theorems [1.3, 1.8, and 1.11 are sharpest for the parabolic Anderson model, particularly when $d=1$. However, an inspection of Theorems 1.3 and 1.8 reveals an inconsistency: Our upper bound on the Liapounov exponent [Theorem 1.3 does not require the drift $b$ to be zero, whereas our lower bound [Theorem 1.8 does. In this connection, David Nualart has asked us whether we know how the drift $b$ can affect the weak intermittence of the solution to (1.1). This seems to be a hard question when the drift $b$ is a general Lipschitz-continuous function, though it is intuitively clear that a sufficiently-strong drift ought to destroy the natural tendency of the solution to be weakly intermittent.

Although we are not aware of general theorems of this type, we are able to give a partial answer to Professor Nualart's question, and the striking nature of our partial answer confirms our initial suspicion that it might be rather difficult to answer such questions in good generality.

Here is an instance where we can rigorously study weak intermittency in the presence of a drift: Consider the one-dimensional parabolic Anderson model for the relativistic [or massive/dissipative] Laplacian; i.e., the stochastic PDE

$$
\frac{\partial}{\partial t} u_{t}(x)=\left(\Delta u_{t}\right)(x)+\frac{\lambda}{2} u_{t}(x)+\kappa u_{t}(x) \dot{F}_{t}(x),
$$

where $t>0$ and $x \in \mathbf{R}, \kappa \neq 0, \lambda \in \mathbf{R}$, and $u_{0}: \mathbf{R} \rightarrow \mathbf{R}$ is a measurable function that is bounded uniformly away from zero and infinity. Let us consider the special case where the correlation function of the noise is of Riesz type; that is,

$$
f(z):=\|z\|^{-1+b} \quad \text { for all } z \in \mathbf{R},
$$


where $b \in(0,1)$. Then, Example 5.8 below implies that weak intermittence is equivalent to the condition

$$
\begin{aligned}
\lambda & >-|\kappa|^{4 /(1+b)} 8^{-(1-b) /(1+b)}\left[\frac{\Gamma(b / 2) \Gamma((b+1) / 2)}{\sqrt{\pi}}\right]^{2 /(1+b)} \\
& =-\left[\frac{\kappa^{4}[\Gamma(b)]^{2}}{2^{1-b}}\right]^{1 /(1+b)} .
\end{aligned}
$$

The inequality is new; the identity follows from a standard application of Euler's duplication formula, $\Gamma(2 z)=(2 \pi)^{-1 / 2} 2^{2 z-(1 / 2)} \Gamma(z) \Gamma\left(z+\frac{1}{2}\right)$, for gamma functions.

A brief outline of the paper follows: In $₫ 2$ we review, very briefly, some analytical facts about Lévy processes and their $L^{2}$ generators, and also construct examples that will be used in subsequent sections.

Section 3 is concerned with positive-definite functions and their connections to potential theory and harmonic analysis. The maximum principle [Theorem 1.2 is derived in $\$ 3$ as a consequence of the mentioned connections. Section 3 also contains a probabilistic characterization of the analytic condition (1.10) and Condition 1.1] in terms of continuous additive functionals of the replica process $\bar{X}$. Those results are analogous to the local-time results of [46, 45] on equation (1.1) driven by space-time white noise. Finally, a family of useful correlation functions is constructed in that section; that construction uses the results of $\$ 2$ on probabilistic potential theory.

In $\$$ we study the linearization of the heat equation (1.1) and derive necessary and sufficient conditions for the existence and spatial continuity of a solution. We also consider various examples, one of which leads to (1.18).

Theorems 1.3 and 1.8 are derived in \$5. In that section we also consider a relativistic version of the stochastic heat equation, thereby constructing examples that include the mentioned analysis of (1.20).

Let us conclude the present section by introducing some notation that will be used throughout the paper. For all integers $k \geq 1$,

$$
\|x\|:=\left(x_{1}^{2}+\cdots+x_{k}^{2}\right)^{1 / 2} \quad \text { for every } x \in \mathbf{R}^{k} .
$$

If $g: \mathbf{R}^{k} \rightarrow \mathbf{R}$ is a function, then

$$
\operatorname{Lip}_{g}:=\sup _{\substack{x, y \in \mathbf{R}^{k} \\ x \neq y}} \frac{|g(x)-g(y)|}{\|x-y\|} .
$$

This so-called Lipschitz constant of $g$ is well defined, but might be infinity.

Throughout this paper, " " denotes the Fourier transform, in the sense of distributions, normalized so that

$$
\hat{g}(\xi):=\int_{\mathbf{R}^{d}} \mathrm{e}^{i x \cdot \xi} g(x) \mathrm{d} x \quad \text { for all } \xi \in \mathbf{R}^{d} \text { and } g \in L^{1}\left(\mathbf{R}^{d}\right) .
$$

For every distribution $v$ on $\mathbf{R}^{d}$ we define the reflection $\tilde{v}$ as the distribution whose pairings are given by $\tilde{v}(\phi):=v(\tilde{\phi})$ for all test functions $\phi$, where $\tilde{\phi}(x):=\phi(-x)$. Note in particular that if $v$ is a Borel measure, then $\tilde{v}(A)=v(-A)$.

\section{LÉVY PROCESSES}

2.1. Preliminaries. Throughout this paper, $X:=\left\{X_{t}\right\}_{t \geq 0}$ denotes a Lévy process on $\mathbf{R}^{d}$. We use notation that is more or less standard and can be found in pedagogic form in 9 , 58, 65, 85]. 
Let $m_{t}$ denote the distribution of $X_{t}$ for every $t \geq 0$; that is,

$$
m_{t}(A):=\mathrm{P}\left\{X_{t} \in A\right\} \quad \text { for all } t \geq 0 \text { and Borel sets } A \subseteq \mathbf{R}^{d} .
$$

Let us recall that throughout this paper we are assuming that the process $X$ has transition functions; that is,

$$
m_{t}(\mathrm{~d} x) \ll \mathrm{d} x \quad \text { for all } t>0 .
$$

According to Hawkes [54, Theorem 2.2], we can [and will] always select a version of these transition functions that has the following regularity properties:

(1) $\int_{A} p_{t}(z) \mathrm{d} z=m_{t}(A)$ for all $t>0$ and Borel sets $A \subseteq \mathbf{R}^{d}$;

(2) $(0, \infty) \times \mathbf{R}^{d} \ni(t, x) \mapsto p_{t}(x) \in \mathbf{R}_{+}$is Borel measurable;

(3) $x \mapsto p_{t}(x)$ is lower semicontinuous for all $t>0$;

(4) $p_{t+s}(x)=\left(p_{t} * p_{s}\right)(x)$ for all $s, t>0$ and $x \in \mathbf{R}^{d}$;

where "*" denotes the convolution operator. Note that

$$
\mathrm{E} \phi\left(x+X_{t}\right)=\int_{\mathbf{R}^{d}} \phi(z) p_{t}(z-x) \mathrm{d} z=\left(\phi * \tilde{p}_{t}\right)(x),
$$

for every $t \geq 0$ and $x \in \mathbf{R}^{d}$, and for Borel-measurable functions $\phi: \mathbf{R}^{d} \rightarrow \mathbf{R}_{+}$.

The semigroup $\left\{P_{t}\right\}_{t \geq 0}$ of $X$ is defined via

$$
\left(P_{t} \phi\right)(x):=\mathrm{E} \phi\left(x+X_{t}\right) .
$$

It is easy to verify that $\left\{P_{t}\right\}_{t>0}$ is a Feller semigroup; i.e., $P_{t}: C_{0}\left(\mathbf{R}^{d}\right) \rightarrow C_{0}\left(\mathbf{R}^{d}\right)$, where $C_{0}\left(\mathbf{R}^{d}\right)$ denotes the collection of all continuous functions $g: \mathbf{R}^{d} \rightarrow \mathbf{R}$ that vanish at infinity. In fact, under the present conditions, $\left\{P_{t}\right\}_{t \geq 0}$ is strong Feller in the sense of Girsanov [51]; see [54]. We emphasize that $P_{t} \phi=\phi * \tilde{p}_{t}$.

Let $\left\{R_{\alpha}\right\}_{\alpha \geq 0}$ denote the resolvent of $\left\{P_{t}\right\}_{t \geq 0}$; i.e.,

$$
R_{\alpha}:=\int_{0}^{\infty} \mathrm{e}^{-\alpha s} P_{s} \mathrm{~d} s
$$

It follows that if $\phi: \mathbf{R}^{d} \rightarrow \mathbf{R}_{+}$is Borel measurable, then

$$
\left(R_{\alpha} \phi\right)(x)=\int_{0}^{\infty} \phi(z) r_{\alpha}(x-z) \mathrm{d} z=\left(\phi * \tilde{r}_{\alpha}\right)(x),
$$

where

$$
r_{\alpha}(x):=\int_{0}^{\infty} \mathrm{e}^{-\alpha t} p_{t}(x) \mathrm{d} t \quad \text { for } \alpha \geq 0 \text { and } x \in \mathbf{R}^{d} .
$$

Each " $\alpha$-potential density" $r_{\alpha}(x)$ is well defined, but could well be infinity at some [even all, when $\alpha=0$ ] $x \in \mathbf{R}^{d}$. Nevertheless, the regularity properties of the transition functions imply that every $r_{\alpha}$ is lower semicontinuous. Furthermore,

$$
R_{\alpha}: C_{0}\left(\mathbf{R}^{d}\right) \rightarrow C_{0}\left(\mathbf{R}^{d}\right) \quad \text { for every } \alpha>0 .
$$

$R_{\alpha}\left(C_{0}\left(\mathbf{R}^{d}\right)\right)$ is uniformly dense in $C_{0}\left(\mathbf{R}^{d}\right)$ when $\alpha>0$ [13, Exercise (9.13), p. 51].

The characteristic exponent of the process $X$ is a function $\Psi: \mathbf{R}^{d} \rightarrow \mathbf{C}$ that is uniquely defined via the "Lévy-Khintchine formula [9, Theorem 1.2, p. 13]",

$$
\mathrm{Ee}^{i \xi \cdot X_{t}}=\mathrm{e}^{-t \Psi(\xi)} \quad\left(\xi \in \mathbf{R}^{d}, t \geq 0\right) .
$$


2.2. The generator. We will be working with the $L^{2}$-theory of generators, as developed, for instance, in Fukushima et al. 47] for more general Markov processes. We outline the details in the present special case; matters are greatly simplified and in some cases generalized because of harmonic analysis.

Define

$$
\operatorname{Dom}[\mathcal{L}]=\left\{\phi \in L^{2}\left(\mathbf{R}^{d}\right): \Psi \hat{\phi} \in L^{2}\left(\mathbf{R}^{d}\right)\right\} .
$$

Plancherel's theorem guarantees that $\phi \in \operatorname{Dom}[\mathcal{L}]$ if and only if $\phi: \mathbf{R}^{d} \rightarrow \mathbf{R}$ is Borel-measurable, locally integrable, and $\int_{\mathbf{R}^{d}}\left(1+|\Psi(\xi)|^{2}\right)|\hat{\phi}(\xi)|^{2} \mathrm{~d} \xi<\infty$.

It is well known [15. (3.4.14), p. 67] that the following holds:

$$
|\Psi(\xi)|=O\left(\|\xi\|^{2}\right) \quad(\|\xi\| \rightarrow \infty) .
$$

Recall that $W^{1,2}\left(\mathbf{R}^{d}\right)$ denotes the collection of all functions $\phi \in L^{2}\left(\mathbf{R}^{d}\right)$ such that $\nabla \phi \in L^{2}\left(\mathbf{R}^{d}\right)$. It is possible to prove that because of Plancherel's theorem and (2.11), $W^{1,2}\left(\mathbf{R}^{d}\right) \subseteq \operatorname{Dom}[\mathcal{L}] \subseteq L^{2}\left(\mathbf{R}^{d}\right)$ densely.

According to Plancherel's theorem,

$$
\left(\psi, P_{t} \phi\right)_{L^{2}\left(\mathbf{R}^{d}\right)}=\left(\psi * m_{t}, \phi\right)_{L^{2}\left(\mathbf{R}^{d}\right)}=\frac{1}{(2 \pi)^{d}} \int_{\mathbf{R}^{d}} \overline{\hat{\phi}(\xi)} \hat{\psi}(\xi) \mathrm{e}^{-t \Psi(\xi)} \mathrm{d} \xi
$$

for all $t \geq 0$ and $\psi, \phi \in L^{2}\left(\mathbf{R}^{d}\right)$. It follows easily from this and (2.10) that

$$
\mathcal{L} \phi:=\lim _{t \downarrow 0}\left(\frac{P_{t} \phi-\phi}{t}\right)
$$

exists in $L^{2}\left(\mathbf{R}^{d}\right)$ if and only if $\phi \in \operatorname{Dom}[\mathcal{L}]$. Thus, we have the so-called generator $\left[L^{2}\right.$-generator, in fact $] \mathcal{L}$, defined on its domain $\operatorname{Dom}[\mathcal{L}]$. In addition, $\mathcal{L}$ can be thought of as a convolution operator with Fourier multiplier $\hat{\mathcal{L}}=-\tilde{\Psi}$. More precisely,

$$
\widehat{\mathcal{L} \phi}(\xi)=-\Psi(-\xi) \hat{\phi}(\xi) \text { for all } \phi \in \operatorname{Dom}[\mathcal{L}] \text { and } \xi \in \mathbf{R}^{d} .
$$

Let us note that for all $t \geq 0, \xi \in \mathbf{R}^{d}$, and $\phi \in L^{1}\left(\mathbf{R}^{d}\right)$,

$$
\left|\widehat{P_{t} \phi}(\xi)\right|^{2}=\mathrm{e}^{-2 t \operatorname{Re} \Psi(\xi)} \cdot|\hat{\phi}(\xi)|^{2} .
$$

Therefore, the well-known nonnegativity of $\operatorname{Re} \Psi(\xi)$ [see (2.17)] implies that $P_{t}[$ resp. $\left.\alpha R_{\alpha}\right]$ is nonexpansive on $W^{1,2}\left(\mathbf{R}^{d}\right)$ for all $t \geq 0$ [resp. $\alpha>0$ ].

2.3. The replica semigroup and associated Sobolev spaces. Let $X^{*}$ denote an independent copy of the Lévy process $-X$ and, following Lévy [66], define

$$
\bar{X}_{t}:=X_{t}+X_{t}^{*} \quad \text { for all } t \geq 0 .
$$

It is easy to see that $X^{*}:=\left\{X_{t}^{*}\right\}_{t \geq 0}$ is the dual process to $X$ and that $\bar{X}:=\left\{\bar{X}_{t}\right\}_{t \geq 0}$ is a symmetric Lévy process on $\mathbf{R}^{d}$. If we denote the distribution of $\bar{X}_{t}$ by $\bar{m}_{t}$, then $\bar{m}_{t}(A)=\left(m_{t} * \tilde{m}_{t}\right)(A)$ for all Borel sets $A \subseteq \mathbf{R}^{d}$. Because $\widehat{\bar{m}}_{t}(\xi)=\left|\hat{m}_{t}(\xi)\right|^{2}=$ $\mathrm{e}^{-2 t \operatorname{Re} \Psi(\xi)}$ is nonnegative, we deduce the classical fact that

$$
\operatorname{Re} \Psi(\xi) \geq 0 \text { for all } \xi \in \mathbf{R}^{d} .
$$

The absolute-continuity condition (2.2) implies that every $\bar{m}_{t}$ is absolutely continuous with respect to the Lebesgue measure on $\mathbf{R}^{d}[t>0]$. We denote the resulting transition density by $\bar{p}_{t}$. Every $\bar{p}_{t}$ is a symmetric function on $\mathbf{R}^{d}[t>0]$. 
We can always choose a version of $\bar{p}$ that has good regularity features [of the type mentioned earlier for $p$ ]. In fact, the following version works:

$$
\bar{p}_{t}(x):=\left(p_{t} * \tilde{p}_{t}\right)(x)=\int_{\mathbf{R}^{d}} p_{t}(x+z) p_{t}(z) \mathrm{d} z \quad \text { for } x \in \mathbf{R}^{d} \text { and } t>0 .
$$

Equivalently, if $\bar{P}:=\left\{\bar{P}_{t}\right\}_{t \geq 0}$ denotes the semigroup of $\bar{X}$, then

$$
\bar{P}_{t}=P_{t} P_{t}^{*} \quad \text { for all } t \geq 0 \text {, }
$$

where $P_{t}^{*}$ denotes the adjoint of $P_{t}$ in $L^{2}\left(\mathbf{R}^{d}\right)$. Every $\bar{P}_{t}$ is a self-adjoint contraction on $L^{2}\left(\mathbf{R}^{d}\right)$.

Motivated by [59, we refer to $\bar{X}$ and $\bar{P}$ respectively as the replica process and the replica semigroup. The corresponding generator is denoted by $\overline{\mathcal{L}}$ and its domain by $\operatorname{Dom}[\overline{\mathcal{L}}]$.

For all $\alpha \geq 0$, we can define the replica $\alpha$-potential density $\bar{r}_{\alpha}$ as

$$
\bar{r}_{\alpha}(x):=\int_{0}^{\infty} \mathrm{e}^{-\alpha s} \bar{p}_{s}(x) \mathrm{d} s \quad \text { for all } x \in \mathbf{R}^{d} .
$$

Clearly, $\bar{r}_{\alpha}(x)$ is well defined, but $\bar{r}_{\alpha}(x)$ can be infinite for some [and even all, in the case that $\alpha=0] x \in \mathbf{R}^{d}$. The resolvent $\bar{R}:=\left\{\bar{R}_{\alpha}\right\}_{\alpha>0}$ of the semigroup $\bar{P}$ can also be defined as follows:

$$
\left(\bar{R}_{\alpha} \phi\right)(x):=\int_{0}^{\infty} \mathrm{e}^{-\alpha s}\left(\bar{P}_{s} \phi\right)(x) \mathrm{d} s=\int_{\mathbf{R}^{d}} \phi(z) \bar{r}_{\alpha}(z-x) \mathrm{d} z,
$$

for all $\alpha>0$ and $x \in \mathbf{R}^{d}$. Since $\bar{r}_{\alpha}$ is a symmetric function on $\mathbf{R}^{d}$, it follows that $\bar{R}_{\alpha} \phi=\phi * \bar{r}_{\alpha}$. The displayed quantity is called the $\alpha$-potential of $\phi$, and is well defined for example if $\phi: \mathbf{R}^{d} \rightarrow \mathbf{R}_{+}$is Borel measurable, or when $\phi \in L^{p}\left(\mathbf{R}^{d}\right)$ for some $p \in[1, \infty]$, because every $\bar{P}_{s}$ is nonexpansive on $L^{p}\left(\mathbf{R}^{d}\right)$.

2.4. On the heat equation and transition functions. We begin by recalling some generally known facts about the fundamental weak solution to the heat equation for $\mathcal{L}$ : We seek to find a function $H$ such that for all $t>0$ and $x \in \mathbf{R}^{d}$,

$$
\mid \begin{aligned}
& \frac{\partial}{\partial t} H_{t}(x)=\left(\mathcal{L} H_{t}\right)(x), \\
& H_{0}=\delta_{z},
\end{aligned}
$$

where $z \in \mathbf{R}^{d}$ is fixed. Because of (2.14), we can rewrite the preceding as

$$
\mid \begin{aligned}
& \frac{\partial}{\partial t} \hat{H}_{t}(\xi)=-\Psi(\xi) \hat{H}_{t}(\xi), \\
& \hat{H}_{0}(\xi)=\mathrm{e}^{i \xi \cdot z}
\end{aligned}
$$

whose solution is $\hat{H}_{t}(\xi)=\exp \{i \xi \cdot z-t \Psi(\xi)\}$. Direct inspection of the Fourier transform reveals that $H_{t}(x)=p_{t}(z-x)$. Thus, we find that the fundamental solution to (2.22) is the measurable function $(0, \infty) \times \mathbf{R}^{d} \times \mathbf{R}^{d} \ni(t ; x, y) \mapsto$ $p_{t}(y-x)$.

The preceding argument also shows that (2.22) has a function solution if and only if the underlying Lévy process $X$ has transition densities. We are thus led to the natural question: "What are the necessary and sufficient conditions on the characteristic exponent $\Psi$ that ensure the existence of transition densities of the corresponding Lévy processes?" Unfortunately, there is no satisfactorily known answer to this question at this time, though several attempts have been made [11, 43, 52, 90, 89, 88, see also [2, 80] and 95, Example (4.6)]. 
We will use the following unpublished result of Hawkes, which provides a goodenough sufficient condition for the existence of transition functions. We include a proof in order to document this interesting fact.

Proposition 2.1 (Hawkes [55]). The following conditions are equivalent:

(1) Condition (2.2) holds and $p_{t} \in L^{2}\left(\mathbf{R}^{d}\right)$ for all $t>0$.

(2) Condition (2.2) holds and $p_{t} \in L^{\infty}\left(\mathbf{R}^{d}\right)$ for all $t>0$.

(3) Condition (2.2) holds and $p_{t} \in L^{2}\left(\mathbf{R}^{d}\right)$ for almost every $t>0$.

(4) Condition (2.2) holds and $p_{t} \in L^{\infty}\left(\mathbf{R}^{d}\right)$ for almost every $t>0$.

(5) $\exp (-\operatorname{Re} \Psi) \in L^{t}\left(\mathbf{R}^{d}\right)$ for all $t>0$.

(6) $\exp (-\operatorname{Re} \Psi) \in L^{t}\left(\mathbf{R}^{d}\right)$ for almost every $t>0$.

Moreover, any one of these conditions implies that: $(i)(t, x) \mapsto p_{t}(x)$ has a continuous version which is uniformly continuous for all $(t, x) \in[\eta, \infty) \times \mathbf{R}^{d}$ for every $\eta>0$; (ii) $p_{t}$ vanishes at infinity for all $t>0$.

Proof. (Hawkes [55]). Recall that $\int_{\mathbf{R}^{d}} p_{t}(x) \mathrm{d} x=1$ and $p_{t}=p_{t / 2} * p_{t / 2}$. Therefore, two applications of Young's inequality yield $\left\|p_{t}\right\|_{L^{\infty}\left(\mathbf{R}^{d}\right)} \leq\left\|p_{t / 2}\right\|_{L^{2}\left(\mathbf{R}^{d}\right)}^{2} \leq$ $\left\|p_{t / 2}\right\|_{L^{\infty}\left(\mathbf{R}^{d}\right)}$ for all $t>0$. Consequently, (1) $\Leftrightarrow(2)$ and (3) $\Leftrightarrow(4)$.

Next let us suppose that (6) holds. Because $\left|\hat{p}_{t}(\xi)\right|=\left|\mathrm{e}^{-t \Psi(\xi)}\right| \leq \mathrm{e}^{-t \operatorname{Re} \Psi(\xi)}$, Plancherel's theorem ensures that

$$
\left\|p_{t}\right\|_{L^{2}\left(\mathbf{R}^{d}\right)}^{2}=\frac{1}{(2 \pi)^{d}}\left\|\hat{p}_{t}\right\|_{L^{2}\left(\mathbf{R}^{d}\right)}^{2} \leq\left\|\mathrm{e}^{-2 t \operatorname{Re} \Psi}\right\|_{L^{1}\left(\mathbf{R}^{d}\right)} .
$$

Since $\operatorname{Re} \Psi \geq 0$, it follows from (6) that $p_{t} \in L^{2}\left(\mathbf{R}^{d}\right)$ for every $t>0$; i.e., (6) $\Rightarrow$ (1) . Moreover, we have - in this case - the following inversion formula: For almost all $x \in \mathbf{R}^{d}$ and every $t>0$,

$$
p_{t}(x)=\frac{1}{(2 \pi)^{d}} \int_{\mathbf{R}^{d}} \mathrm{e}^{-i \xi \cdot x-t \Psi(\xi)} \mathrm{d} \xi .
$$

It remains to prove that (11) and equivalently (2) together imply (5). We may observe that the Fourier transform of $p_{t / 4} * \tilde{p}_{t / 4}$ is $\exp \{-(t / 2) \operatorname{Re} \Psi\}$. Therefore, by Plancherel's theorem,

$$
\left\|\mathrm{e}^{-\operatorname{Re} \Psi}\right\|_{L^{t}\left(\mathbf{R}^{d}\right)}=(2 \pi)^{d}\left\|p_{t / 4} * \tilde{p}_{t / 4}\right\|_{L^{2}\left(\mathbf{R}^{d}\right)}^{2} \leq(2 \pi)^{d}\left\|p_{t / 4} * \tilde{p}_{t / 4}\right\|_{L^{\infty}\left(\mathbf{R}^{d}\right)} .
$$

Consequently, Young's inequality implies that $\left\|\mathrm{e}^{-\operatorname{Re} \Psi}\right\|_{L^{t}\left(\mathbf{R}^{d}\right)} \leq(2 \pi)^{d}\left\|p_{t / 4}\right\|_{L^{2}\left(\mathbf{R}^{d}\right)}^{2}$, which has the desired effect.

Finally, if (5) holds, then the inversion theorem applies and tells us that we can always choose a version of $p$ that satisfies the properties of the final paragraph in the statement of the theorem.

2.5. On a family of isotropic Lévy processes. The main result of this section will be needed to construct a counterexample in $\S 4$. It is possible that it is known, but we were not able to find an explicit reference. Therefore, we provide a complete proof. We begin by recalling a few definitions used to study Lévy processes.

We say that a Lévy process $X:=\left\{X_{t}\right\}_{t \geq 0}$ is isotropic - or radial [76] -if its characteristic exponent $\Psi$ is a radial function [and hence also real-valued and nonnegative].

A [standard] subordinator $\tau:=\left\{\tau_{t}\right\}_{t \geq 0}$ is a one-dimensional Lévy process that is nondecreasing and $\tau_{0}:=0$. According to the Lévy-Khintchine formula 10 , 
Theorem 1.2, p. 13], every subordinator $\tau$ is determined by the formula $\mathrm{Ee}^{-\lambda \tau_{t}}=$ $\mathrm{e}^{-t \Phi(\lambda)}$, where $t, \lambda \geq 0$, and

$$
\Phi(\lambda)=\int_{0}^{\infty}\left(1-\mathrm{e}^{-\lambda z}\right) \Pi(\mathrm{d} z)
$$

for a Borel measure $\Pi$ on $\mathbf{R}_{+}$- the so-called Lévy measure - that satisfies the conditions

$$
\Pi(\{0\})=0, \int_{0}^{\infty}(1 \wedge x) \Pi(\mathrm{d} x)<\infty .
$$

The function $\Phi$ is the so-called Laplace exponent of the subordinator $\tau$.

We have the following lemma.

Lemma 2.2. Choose and fix two numbers $p \in(0,1)$ and $q \in \mathbf{R}$. Then, there exists a subordinator $\tau$ on $\mathbf{R}_{+}$whose Laplace exponent satisfies

$$
0<\inf _{\lambda>\mathrm{e}} \frac{\Phi(\lambda)}{\lambda^{p}(\log \lambda)^{q / 2}} \leq \sup _{\lambda>\mathrm{e}} \frac{\Phi(\lambda)}{\lambda^{p}(\log \lambda)^{q / 2}}<\infty .
$$

Proof. Define a measure $\Pi$ via

$$
\frac{\Pi(\mathrm{d} x)}{\mathrm{d} x}:= \begin{cases}x^{-1-p}(\log (1 / x))^{q / 2} & \text { if } 0<x<\frac{1}{2} \\ 0 & \text { otherwise. }\end{cases}
$$

It is easy to see that $\Pi$ is a bona fide Lévy measure because $p \in(0,1)$.

We apply the definition (2.27) of the Laplace exponent and write $\Phi(\lambda)=\lambda^{p} Q(\lambda)$, where

$$
Q(\lambda)=\int_{0}^{\lambda / 2} \frac{1-\mathrm{e}^{-x}}{x^{1+p}}(\log (\lambda / x))^{q / 2} \mathrm{~d} x \quad \text { for } \lambda>0 .
$$

In order to complete the proof, we will verify that $Q(\lambda) \asymp(\log \lambda)^{q / 2}$ for $\lambda>\mathrm{e} 1$ We do so in the special case that $q \geq 0$; similar arguments can be used to estimate $Q(\lambda)$ in the case that $q<0$.

Whenever $\lambda>$ e, we can write $Q(\lambda):=I_{1}+I_{2}$, where

$$
\begin{aligned}
& I_{1}:=\int_{1}^{\lambda / 2} \frac{1-\mathrm{e}^{-x}}{x^{1+p}}(\log (\lambda / x))^{q / 2} \mathrm{~d} x, \\
& I_{2}:=\int_{0}^{1} \frac{1-\mathrm{e}^{-x}}{x^{1+p}}(\log (\lambda / x))^{q / 2} \mathrm{~d} x .
\end{aligned}
$$

Evidently,

$$
I_{1} \leq(\log \lambda)^{q / 2} \cdot \int_{1}^{\infty} \frac{\mathrm{d} x}{x^{1+p}}=\frac{1}{p}(\log \lambda)^{q / 2} .
$$

Since $I_{1} \geq 0$, it remains to prove that $I_{2} \asymp(\log \lambda)^{q / 2}$ for $\lambda>1$. We establish this by deriving first an upper, and then a lower, bound for $I_{2}$. Because $1-\exp (-y) \leq y$ for $y \geq 0$, and since $\sup _{z \in(0,1)} z^{\epsilon} \log (1 / z)<\infty$ for all $\epsilon \in(0,1)$, it follows that

$$
I_{2} \leq(\log \lambda)^{q / 2} \cdot \int_{0}^{1}\left(1+\frac{\log (1 / x)}{\log \lambda}\right)^{q / 2} \frac{\mathrm{d} x}{x^{p}} \leq \text { const } \cdot(\log \lambda)^{q / 2} .
$$

\footnotetext{
${ }^{1}$ As usual, $h(x) \asymp g(x)$ over a certain range of $x$ 's is shorthand for the statement that, uniformly over that range of $x$ 's, $h(x) / g(x)$ is bounded above and below by positive and finite constants.
} 
A similar lower bound is obtained via the bounds: (i) $1-\exp (-x) \geq x / 2$; (ii) $\log (\lambda / x) \geq \log \lambda$; both are valid for all $x \in(0,1)$.

The following is the main result of this section. It gives a special construction of an isotropic Lévy process $X:=\left\{X_{t}\right\}_{t \geq 0}$ whose characteristic exponent is regularly varying in a special manner.

Theorem 2.3. Choose and fix $r \in(0,2)$ and $q \in \mathbf{R}$. Then, there exists an isotropic Lévy process $X:=\left\{X_{t}\right\}_{t \geq 0}$ such that

$$
0<\inf _{\substack{\xi \in \mathbf{R}^{d}: \\\|\xi\|>\mathrm{e}}} \frac{\Psi(\xi)}{\|\xi\|^{r}(\log \|\xi\|)^{q}} \leq \sup _{\substack{\xi \in \mathbf{R}^{d}: \\\|\xi\|>\mathrm{e}}} \frac{\Psi(\xi)}{\|\xi\|^{r}(\log \|\xi\|)^{q}}<\infty .
$$

Proof. Let $B:=\{B(t)\}_{t \geq 0}$ denote a $d$-dimensional Brownian motion, independent from the subordinator $\tau:=\left\{\tau_{t}\right\}_{t \geq 0}$ of Lemma 2.2. Define $X_{t}:=B\left(\tau_{t}\right)$ for all $t \geq 0$. It is well known - as well as easy to check - that the process $X:=\left\{X_{t}\right\}_{t \geq 0}$ is a Lévy process with characteristic exponent $\Psi(\xi)=\Phi\left(\|\xi\|^{2} / 2\right)$. We can apply Lemma 2.2 with $p:=r / 2$ to complete the remainder of the proof.

\section{Positive-Definite Functions and Probabilistic Potential theory}

A large part of this paper relies heavily on our ensuing analysis of positivedefinite functions and their many connections to harmonic analysis. In this section we develop the requisite theory and then derive Theorem 1.2 as a consequence. We also provide intrinsically-probabilistic interpretations of the two central potentialtheoretic hypotheses of this paper, namely Conditions 1.1 and 1.10

3.1. Fourier analysis. Our normalization (1.25) of Fourier transforms ensures the following form of the Parseval identity:

$$
\int_{\mathbf{R}^{d}} g(x) h(x) \mathrm{d} x=\frac{1}{(2 \pi)^{d}} \int_{\mathbf{R}^{d}} \hat{g}(\xi) \overline{\hat{h}(\xi)} \mathrm{d} \xi .
$$

The preceding is valid when $g, h \in L^{2}\left(\mathbf{R}^{d}\right)$ and also when $g \in \mathcal{S}$-the collection of all rapidly-decreasing tests functions - and $h: \mathbf{R}^{d} \rightarrow \mathbf{R}$ is continuous and tempered [say]. It might help to recall that $h: \mathbf{R}^{d} \rightarrow \mathbf{R}$ is tempered if it is Borel-measurable and there exists $k \geq 0$ such that $|h(x)|=O\left(|x|^{k}\right)$ as $|x| \rightarrow \infty$.

3.2. Positive-definite functions. Recall that a function $g: \mathbf{R}^{d} \rightarrow \mathbf{R}_{+}$is positive definite if $g$ is tempered and $(\phi, g * \phi)_{L^{2}\left(\mathbf{R}^{d}\right)} \geq 0$ for all rapidly-decreasing test functions $\phi$. A theorem of L. Schwartz [50, Theorem 3, p. 157] tells us that $g$ : $\mathbf{R}^{d} \rightarrow \overline{\mathbf{R}}$ is positive definite if and only if $g=\hat{\Gamma}$ for a tempered Borel measure $\Gamma$ on $\mathbf{R}^{d}$. Schwartz's theorem is a generalization of the following theorem of Herglotz $[d=1]$ and Bochner $[d \geq 2]:$ If $g: \mathbf{R}^{d} \rightarrow \mathbf{R}$ is continuous and positive definite, then there exists a finite Borel measure $\Gamma$ on $\mathbf{R}^{d}$ such that $g=\hat{\Gamma}$.

3.3. A preliminary maximum principle. Now that we have recalled the basic definitions and properties of positive-definite functions, we can begin our proof of our maximum principle [Theorem 1.2]. But first let us prove the following technical result.

Lemma 3.1. If $\phi \in \mathcal{S}$, then there exists a version of $f * \phi$ that is in $C_{0}\left(\mathbf{R}^{d}\right)$. Consequently, $\bar{R}_{\beta}(f * \phi) \in C_{0}\left(\mathbf{R}^{d}\right)$ for every $\beta>0$. 
Proof. Because $\hat{f}$ is tempered, the following defines a uniformly continuous function on $\mathbf{R}^{d}$ :

$$
h(x)=\frac{1}{(2 \pi)^{d}} \int_{\mathbf{R}^{d}} \mathrm{e}^{-i x \cdot \xi} \hat{f}(\xi) \hat{\phi}(\xi) \mathrm{d} \xi \quad \text { for all } x \in \mathbf{R}^{d} .
$$

In fact, $h \in C_{0}\left(\mathbf{R}^{d}\right)$ because of the Riemann-Lebesgue lemma. Furthermore, if $\psi \in \mathcal{S}$, then

$$
\int_{\mathbf{R}^{d}} \psi(x) h(x) \mathrm{d} x=\frac{1}{(2 \pi)^{d}} \int_{\mathbf{R}^{d}} \overline{\hat{\psi}(\xi)} \hat{f}(\xi) \hat{\phi}(\xi) \mathrm{d} \xi=\int_{\mathbf{R}^{d}} \psi(x)(f * \phi)(x) \mathrm{d} x .
$$

The first line is justified by the Fubini theorem, and the second by the Parseval identity. It follows from density and the Lebesgue differentiation theorem that $h=f * \phi$ almost everywhere. This proves the first assertion of the lemma. In addition,

$$
\left(\bar{R}_{\beta} h\right)(x)=\int_{\mathbf{R}^{d}} \bar{r}_{\beta}(y-x)(f * \phi)(y) \mathrm{d} y=\left(\bar{R}_{\beta}(f * \phi)\right)(x) .
$$

Since $h \in C_{0}\left(\mathbf{R}^{d}\right)$, it follows from (2.8) that $\bar{R}_{\beta}(f * \phi)=\bar{R}_{\beta} h \in C_{0}\left(\mathbf{R}^{d}\right)$.

The following contains a portion of the said maximum principle of Theorem 1.2 . It also provides some of the requisite technical estimates that are needed for the remainder of the proof of Theorem 1.2

Proposition 3.2. For all $\beta \geq 0$,

$$
\Upsilon(\beta)=\sup _{x \in \mathbf{R}^{d}}\left(\bar{R}_{\beta} f\right)(x)=\operatorname{ess}_{x \in \mathbf{R}^{d}} \sup \left(\bar{R}_{\beta} f\right)(x)=\limsup _{x \rightarrow 0}\left(\bar{R}_{\beta} f\right)(x),
$$

where $\Upsilon(0):=\lim _{\beta \downarrow 0} \Upsilon(\beta)$.

Proof. First, we prove the proposition in the case where $\beta>0$.

In accordance with Lemma 3.1, if $\phi \in \mathcal{S}$, then $\bar{R}_{\beta}(f * \phi)$ is continuous. Therefore, the Plancherel theorem applies pointwise: For all $x \in \mathbf{R}^{d}$,

$$
\left(\bar{R}_{\beta}(f * \phi)\right)(x)=\frac{1}{(2 \pi)^{d}} \int_{\mathbf{R}^{d}} \frac{\hat{f}(\xi) \hat{\phi}(\xi) \mathrm{e}^{-i x \cdot \xi}}{\beta+2 \operatorname{Re} \Psi(\xi)} \mathrm{d} \xi .
$$

[Without the asserted continuity, we could only deduce this for almost every $x \in$ $\mathbf{R}^{d}$.] In particular, for all probability densities $\phi \in \mathcal{S}$,

$$
\sup _{x \in \mathbf{R}^{d}}\left(\bar{R}_{\beta}(f * \phi)\right)(x) \leq \Upsilon(\beta) .
$$

[ $\Upsilon$ was defined in (1.8).] If $\left\{\phi_{n}\right\}_{n=1}^{\infty}$ is an approximate identity consisting solely of probability densities in $\mathcal{S}$, then $\bar{R}_{\beta} f \leq \liminf _{n \rightarrow \infty} \bar{R}_{\beta}\left(f * \phi_{n}\right)$ pointwise, thanks to Fatou's lemma. Consequently, (3.7) implies that

$$
\sup _{x \in \mathbf{R}^{d}}\left(\bar{R}_{\beta} f\right)(x) \leq \Upsilon(\beta) .
$$

In order to prove the reverse bound, define the Gaussian mollifiers $\left\{\phi_{n}\right\}_{n=1}^{\infty}$ :

$$
\phi_{n}(z):=\left(\frac{n}{2 \pi}\right)^{d / 2} \exp \left(-\frac{\|z\|^{2} n}{2}\right) .
$$

Observe that because of the monotone convergence theorem,

$$
\left(\bar{R}_{\beta}\left(f * \phi_{n}\right)\right)(0)=\frac{1}{(2 \pi)^{d}} \int_{\mathbf{R}^{d}} \frac{\hat{f}(\xi) \mathrm{e}^{-\|\xi\|^{2} /(2 n)}}{\beta+2 \operatorname{Re} \Psi(\xi)} \mathrm{d} \xi=(1+o(1)) \Upsilon(\beta),
$$


as $n \rightarrow \infty$. On the other hand,

$$
\left(\bar{R}_{\beta}\left(f * \phi_{n}\right)\right)(0)=\int_{\mathbf{R}^{d}}\left(\bar{R}_{\beta} f\right)(y) \phi_{n}(y) \mathrm{d} y \leq \underset{x \in \mathbf{R}^{d}}{\operatorname{ess} \sup }\left(\bar{R}_{\beta} f\right)(x),
$$

since $\int_{\mathbf{R}^{d}} \phi_{n}(y) \mathrm{d} y=1$. This and (3.7) together prove that $\Upsilon(\beta)$ is the maximum of the $\beta$-potential of $f$, and the maximum $\beta$-potential is finite if and only if $\Upsilon(\beta)$ is. We can choose the $\phi_{n}$ 's so that in addition to the preceding regularity criteria, every $\phi_{n}$ is supported in the ball of radius $1 / n$ about the origin. In that way we obtain $\left(\bar{R}_{\beta}\left(f * \phi_{n}\right)\right)(0)=\left(\left(\bar{R}_{\beta} f\right) * \phi_{n}\right)(0) \leq \sup _{\|x\|<1 / n}\left(\bar{R}_{\beta} f\right)(x)$. This proves (3.5) for every $\beta \in(0, \infty)$.

Now we address the case where $\beta:=0$. First of all, $\beta \mapsto \Upsilon(\beta)$ is nonincreasing. Therefore, $\Upsilon(0):=\lim _{\beta \downarrow 0} \Upsilon(\beta)$ exists as a nondecreasing limit. Because $\bar{R}_{\beta} f \leq$ $\bar{R}_{0} f$ [pointwise] for all $\beta>0$, we can deduce that $\Upsilon(0) \leq \operatorname{ess} \sup _{x \in \mathbf{R}^{d}}\left(\bar{R}_{0} f\right)(x)$ and $\Upsilon(0) \leq \lim \sup _{x \rightarrow 0}\left(\bar{R}_{0} f\right)(x)$.

For the reverse bound, recall (3.7) and let $\beta \downarrow 0$. Since $\Upsilon(\beta) \rightarrow \Upsilon(0)$, we find that for every probability density $\phi \in \mathcal{S}$ and $x \in \mathbf{R}^{d}$,

$$
\lim _{\beta \downarrow 0}\left(\bar{R}_{\beta}\left(f * \phi_{n}\right)\right)(x) \leq \Upsilon(0) .
$$

But the left-hand side is

$$
\lim _{\beta \downarrow 0} \mathrm{E}\left[\int_{0}^{\infty}\left(f * \phi_{n}\right)\left(\bar{X}_{s}+x\right) \mathrm{e}^{-\beta s} \mathrm{~d} s\right]=\left(\bar{R}_{0}\left(f * \phi_{n}\right)\right)(x),
$$

thanks to the monotone convergence theorem. Another application of Fatou's lemma shows that $\left(\bar{R}_{0} f\right)(x) \leq \Upsilon(0)$ for all $x \in \mathbf{R}^{d}$. This establishes (3.5), and hence the proposition, in the case where $\beta=0$.

3.4. Proof of the maximum principle. The main goal of this subsection is to establish Theorem 1.2. This subsection also contains a harmonic-analytic estimate that might be of independent interest. We will use that harmonic-analytic to demonstrate Theorem 1.2 as well as the subsequent Theorems 1.8 and 1.11.

In order to motivate our estimate, let us first consider the important special case where the correlation function $f$ is of Riesz type. That is,

$$
f(z):=\|z\|^{-(d-b)} \quad \text { for } z \in \mathbf{R}^{d},
$$

where $0^{-1}:=\infty$. Clearly, $f$ is locally integrable when $b \in(0, d)$, a condition which we assume, and in fact its Fourier transform is [73, eq. (12.10), p. 161]

$$
\hat{f}(\xi)=\frac{\pi^{d / 2} 2^{b} \Gamma(b / 2)}{\Gamma((d-b) / 2)} \cdot\|\xi\|^{-b} .
$$

Then, it is well known that

$$
\begin{aligned}
\iint f(x-y) \mu(\mathrm{d} x) \mu(\mathrm{d} y) & =\iint \frac{\mu(\mathrm{d} x) \mu(\mathrm{d} y)}{\|x-y\|^{d-b}} \\
& =\frac{\pi^{d / 2} 2^{b} \Gamma(b / 2)}{\Gamma((d-b) / 2)} \cdot \int_{\mathbf{R}^{d}} \frac{|\hat{\mu}(\xi)|^{2}}{\|\xi\|^{b}} \mathrm{~d} \xi \\
& =\frac{1}{(2 \pi)^{d}} \int_{\mathbf{R}^{d}}|\hat{\mu}(\xi)|^{2} \hat{f}(\xi) \mathrm{d} \xi .
\end{aligned}
$$

It is easy to guess this famous identity from an informal application of the Fubini theorem. However, a rigorous derivation of (3.16) requires a good deal of effort [73. Lemma 12.12 , p. 162]. In the language of potential theory, the preceding 
asserts that the Riesz-type "energy" of the form $\iint\|x-y\|^{-d+b} \mu(\mathrm{d} x) \mu(\mathrm{d} y)$ is equal to a constant multiple of the Pólya-Szegö-type energy $\int_{\mathbf{R}^{d}}|\hat{\mu}(\xi)|^{2}\|\xi\|^{-b} \mathrm{~d} \xi$. The following proposition shows that there is a very general lower bound that is valid for every correlation function $f$.

Proposition 3.3. For all Borel probability measures $\mu$ on $\mathbf{R}^{d}$,

$$
\iint f(x-y) \mu(\mathrm{d} x) \mu(\mathrm{d} y) \geq \frac{1}{(2 \pi)^{d}} \int_{\mathbf{R}^{d}}|\hat{\mu}(\xi)|^{2} \hat{f}(\xi) \mathrm{d} \xi .
$$

Proof. We will assume, without loss of generality, that

$$
\iint f(x-y) \mu(\mathrm{d} x) \mu(\mathrm{d} y)<\infty
$$

for there is nothing to prove otherwise. We also observe that

$$
\iint f(x-y) \mu(\mathrm{d} x) \mu(\mathrm{d} y)=\int(f * \mu) \mathrm{d} \mu .
$$

This is essentially the famous "reciprocity theorem" of classical potential theory.

Because $f * \mu \in L^{1}(\mu)$, Lusin's theorem implies that for all $\epsilon>0$ there exists a compact set $A_{\epsilon}$ in $\mathbf{R}^{d}$ such that: (i) $\mu\left(A_{\epsilon}^{c}\right)<\epsilon$; (ii) $f * \mu$ is continuous on $A_{\epsilon}$.

Let

$$
\mu_{\epsilon}(\bullet):=\mu\left(\bullet \cap A_{\epsilon}\right)
$$

denote the restriction of $\mu$ to $A_{\epsilon}$, and recall the Gaussian densities $\{\phi\}_{n=1}^{\infty}$ from (3.9). Because $\lim _{n \rightarrow \infty}\left(f * \phi_{n / 2} * \mu\right)=f * \mu$ uniformly on $A_{\epsilon}$, it follows from (3.19) that

$$
\begin{aligned}
\iint f(x-y) \mu(\mathrm{d} x) \mu(\mathrm{d} y) \geq \int_{A_{\epsilon}}(f * \mu) \mathrm{d} \mu & =\int(f * \mu) \mathrm{d} \mu_{\epsilon} \\
& =\lim _{n \rightarrow \infty} \int\left(f * \phi_{n / 2} * \mu\right) \mathrm{d} \mu_{\epsilon} \\
& \geq \limsup _{n \rightarrow \infty} \int\left(f * \phi_{n / 2} * \mu_{\epsilon}\right) \mathrm{d} \mu_{\epsilon} .
\end{aligned}
$$

Since $\phi_{n / 2}=\phi_{n} * \phi_{n}$

$$
\iint f(x-y) \mu(\mathrm{d} x) \mu(\mathrm{d} y) \geq \limsup _{n \rightarrow \infty} \int_{\mathbf{R}^{d}}\left(f * \mu_{n, \epsilon}\right)(x) \mu_{n, \epsilon}(x) \mathrm{d} x,
$$

where $\mu_{n, \epsilon}(x):=\left(\phi_{n} * \mu_{\epsilon}\right)(x)$. Since $\mu_{n, \epsilon} \in \mathcal{S}$ and $f * \mu_{n, \epsilon}$ is $C^{\infty}$ and tempered, Parseval's identity (3.1) implies that

$$
\int_{\mathbf{R}^{d}}\left(f * \mu_{n, \epsilon}\right)(x) \mu_{n, \epsilon}(x) \mathrm{d} x=\frac{1}{(2 \pi)^{d}} \int_{\mathbf{R}^{d}} \mathrm{e}^{-\|\xi\|^{2} / n}\left|\hat{\mu}_{\epsilon}(\xi)\right|^{2} \hat{f}(\xi) \mathrm{d} \xi .
$$

This, (3.21), and the monotone convergence theorem together imply that

$$
\iint f(x-y) \mu(\mathrm{d} x) \mu(\mathrm{d} y) \geq \frac{1}{(2 \pi)^{d}} \int_{\mathbf{R}^{d}}\left|\hat{\mu}_{\epsilon}(\xi)\right|^{2} \hat{f}(\xi) \mathrm{d} \xi .
$$

Since $\mu_{\epsilon}$ converges weakly to $\mu$ as $\epsilon \rightarrow 0$, we know that $\hat{\mu}_{\epsilon}$ converges to $\hat{\mu}$ pointwise. The proposition follows from this and Fatou's lemma.

An elementary computation [73, Lemma 12.11, p. 161] and Proposition 3.3 above together yield the following corollary. 
Corollary 3.4. If $f$ is lower semicontinuous, then for all Borel probability measures $\mu$ on $\mathbf{R}^{d}$,

$$
\iint f(x-y) \mu(\mathrm{d} x) \mu(\mathrm{d} y)=\frac{1}{(2 \pi)^{d}} \int_{\mathbf{R}^{d}}|\hat{\mu}(\xi)|^{2} \hat{f}(\xi) \mathrm{d} \xi
$$

A weaker version of Corollary 3.4 can be found in [61, Theorem 5.2].

We are now ready to prove Theorem 1.2 .

Proof of Theorem 1.2. Note that for all $t \geq 0$,

$$
\left(\bar{P}_{t} f\right)(0)=\left(P_{t}^{*} P_{t} f\right)(0)=\left(\tilde{p}_{t} * p_{t} * f\right)(0)=\iint f(x-y) p_{t}(x) p_{t}(y) \mathrm{d} x \mathrm{~d} y .
$$

This requires only the Tonelli theorem. Proposition 3.3 shows us that

$$
\left(\bar{P}_{t} f\right)(0) \geq \frac{1}{(2 \pi)^{d}} \int_{\mathbf{R}^{d}} \mathrm{e}^{-2 t \operatorname{Re} \Psi(\xi)} \hat{f}(\xi) \mathrm{d} \xi .
$$

Multiply both sides by $\exp (-\beta t)$ and integrate $[\mathrm{d} t]$ to find that

$$
\left(\bar{R}_{\beta} f\right)(0) \geq \frac{1}{(2 \pi)^{d}} \int_{\mathbf{R}^{d}} \frac{\hat{f}(\xi)}{\beta+2 \operatorname{Re} \Psi(\xi)} \mathrm{d} \xi=\Upsilon(\beta) .
$$

This and Proposition 3.2 together imply (1.10). Consequently, (1.1) holds if and only if $\Upsilon(\beta)<\infty$ for all $\beta>0$.

Next we prove that $\bar{R}_{\beta} f \in C_{0}\left(\mathbf{R}^{d}\right)$ whenever $\Upsilon(\beta)<\infty$ and $f$ is lower semicontinuous.

When $f$ is lower semicontinuous we can find compactly-supported continuous functions $f_{n}$ that converge upward to $f$ as $n \rightarrow \infty$. Recall from (2.8) that $\bar{R}_{\beta}$ maps $C_{0}\left(\mathbf{R}^{d}\right)$ to $C_{0}\left(\mathbf{R}^{d}\right)$. Consequently, $\bar{R}_{\beta} f_{n} \in C_{0}\left(\mathbf{R}^{d}\right)$, and from this we may conclude that $\bar{R}_{\beta} f$ is lower semicontinuous.

Next, let us define

$$
\pi_{\beta}(x):=\frac{1}{(2 \pi)^{d}} \int_{\mathbf{R}^{d}} \frac{\mathrm{e}^{-i \xi \cdot x} \hat{f}(\xi)}{\beta+2 \operatorname{Re} \Psi(\xi)} \mathrm{d} \xi .
$$

If $\Upsilon(\beta)<\infty$, then $\pi_{\beta} \in C_{0}\left(\mathbf{R}^{d}\right)$. Moreover, a few successive applications of Fubini's theorem tell us that $\int_{\mathbf{R}^{d}} \pi_{\beta}(x) \phi(x) \mathrm{d} x=\int_{\mathbf{R}^{d}}\left(\bar{R}_{\beta} f\right)(x) \phi(x) \mathrm{d} x$ for all $\phi \in \mathcal{S}$. Thus, $\pi_{\beta}=\bar{R}_{\beta} f$ a.e.

It remains to prove that if $\Upsilon(1)$ is finite, then so is $\Upsilon(\beta)$ for every $\beta>0$.

We have shown that if $\Upsilon(1)$ is finite, then $\bar{R}_{1} f=\pi_{1}$ almost everywhere, $\pi_{1} \in$ $C_{0}\left(\mathbf{R}^{d}\right)$; also, $\bar{R}_{1} f$ is bounded [Proposition 3.2. If $h_{1}=h_{2}$ almost everwhere and $h_{1}, h_{2}: \mathbf{R}^{d} \rightarrow \mathbf{R}_{+}$are measurable, then

$$
\left(\bar{R}_{\beta} h_{1}\right)(x)=\int_{\mathbf{R}^{d}} \bar{r}_{\beta}(y) h_{1}(x-y) \mathrm{d} y=\int_{\mathbf{R}^{d}} \bar{r}_{\beta}(y) h_{2}(x-y) \mathrm{d} y=\left(\bar{R}_{\beta} h_{2}\right)(x),
$$

for all $x \in \mathbf{R}^{d}$. Therefore, (2.8) and the fact that $\pi_{1} \in C_{0}\left(\mathbf{R}^{d}\right)$ together imply that $\bar{R}_{\beta} \pi_{1} \in C_{0}\left(\mathbf{R}^{d}\right)$ for all $\beta>0$. In particular, $\bar{R}_{\beta} \bar{R}_{1} f \in C_{0}\left(\mathbf{R}^{d}\right)$-whence $\bar{R}_{\beta} \bar{R}_{1} f$ is bounded-for every $\beta>0$. Using the resolvent equation [13. (8.10), p. 41], the boundedness of $\bar{R}_{1} f$ and $\bar{R}_{\beta} \bar{R}_{1} f$ [for all $\beta>0$ ] together imply that $\bar{R}_{\beta} f$ is bounded for every $\beta>0$. Consequently, Proposition 3.2 implies the finiteness of $\Upsilon(\beta)$ for every $\beta>0$. 
3.5. Probabilistic potential theory. Define, for all measurable functions $\phi$ : $\mathbf{R}^{d} \rightarrow \mathbf{R}_{+}$, a stochastic process $\left\{L_{t}(\phi)\right\}_{t \geq 0}$ as follows:

$$
L_{t}(\phi):=\int_{0}^{t} \phi\left(\bar{X}_{s}\right) \mathrm{d} s \quad(0 \leq t \leq \infty) .
$$

The random field $(t, \phi) \mapsto L_{t}(\phi)$ defined above is often called the occupation field of the Lévy process $\bar{X}$ [41. The occupation field is well defined, though it can be infinite, even in simple cases. The following example highlights this and also paves the way to the ensuing discussion which yields a probabilistic interpretation of Conditions 1.1 and 1.10.

Example 3.5 (After Girsanov, 1962 [74, §3.10, pp. 78-81]). Consider $d=1$, and let $X$ denote 1-D Brownian motion, normalized so that $\operatorname{E} \exp \left(i \xi \cdot X_{t}\right)=\exp \left(-t \xi^{2} / 4\right)$ for all $t>0$ and $\xi \in \mathbf{R}$. In this way, $\bar{X}$ is normalized to be standard linear Brownian motion; that is, $\operatorname{Eexp}\left(i \xi \cdot \bar{X}_{t}\right)=\exp \left(-t \xi^{2} / 2\right)$ for all $t>0$ and $\xi \in \mathbf{R}$. Let $\phi(x):=|x|^{-\alpha}$ for all $x \in \mathbf{R}$, where $\alpha \in(0,1)$ is fixed. By the occupation density formula [84, Cor. (1.6), p. 209],

$$
L_{t}(\phi)=\int_{-\infty}^{\infty} \frac{\ell_{t}^{x}}{|x|^{\alpha}} \mathrm{d} x
$$

where $\ell$ denotes the process of local times associated to $\bar{X}$. According to Trotter's theorem [84, Theorem (1.7), p. 209] and the occupation density formula,

$$
\mathrm{P}\left\{\ell_{t}^{\bullet} \in C_{0}(\mathbf{R}) \text { for all } t>0\right\}=1 .
$$

It is a well-known consequence of the Blumenthal zero-one law and Brownian scaling that $\mathrm{P}\left\{\ell_{t}^{0}>0\right.$ for all $\left.t>0\right\}=1$. Consequently [if we ignore the null sets in the usual way], $L_{t}(\phi)<\infty$ for some -hence all- $t>0$ if and only if $\alpha<1$. At the same time, we note that

$$
\mathrm{E} L_{t}(\phi)=\mathrm{E} \int_{0}^{t} \frac{\mathrm{d} s}{\left|\bar{X}_{s}\right|^{\alpha}}=\int_{0}^{t} \frac{\mathrm{d} s}{s^{\alpha / 2}} \cdot \frac{1}{\sqrt{2 \pi}} \int_{-\infty}^{\infty}|z|^{-\alpha} \mathrm{e}^{-z^{2} / 2} \mathrm{~d} z .
$$

This requires only Brownian scaling and the Tonelli theorem. Thus, $\mathrm{E} L_{t}(\phi)$ is finite for all $t>0$ if and only if $\alpha<1$. In rough terms, we have shown that $L_{t}(\phi)<\infty$ if and only if $\mathrm{E} L_{t}(\phi)<\infty$. As we shall see, a suitable interpretation of this property can be generalized; see Theorem 3.10 below.

It is convenient to use some notation from Markov-process theory: Recall from Markov-process theory that $\mathrm{P}_{z}$ denotes the law of the underlying Lévy process started at $z \in \mathbf{R}^{d}$ [so that $\mathrm{P}=\mathrm{P}_{0}$ ] and that $\mathrm{E}_{z}$ denotes the corresponding expectation operator. Thus,

$$
\left(\bar{R}_{\alpha} \phi\right)(z)=\mathrm{E}_{z} \int_{0}^{\infty} \mathrm{e}^{-\alpha s} \phi\left(\bar{X}_{s}\right) \mathrm{d} s .
$$

Before we state and prove the main result of this section, let us first establish some technical facts which will be needed in the proof of the main result.

Lemma 3.6. For all $t, \alpha>0$ and measurable functions $\phi: \mathbf{R}^{d} \rightarrow \mathbf{R}_{+}$,

$$
\sup _{x \in \mathbf{R}^{d}} \mathrm{E}_{x}\left(\sup _{s>0}\left[\mathrm{e}^{-\alpha s} L_{s}(\phi)\right]\right) \leq \sup _{z \in \mathbf{R}^{d}}\left(\bar{R}_{\alpha} \phi\right)(z) \leq \chi_{\alpha}(t) \sup _{x \in \mathbf{R}^{d}} \mathrm{E}_{x} L_{t}(\phi),
$$

where $\chi_{\alpha}(t):=\mathrm{e}^{\alpha t} /\left(\mathrm{e}^{\alpha t}-1\right)$. 
Remark 3.7. Let us point out the following elementary bound for the right-most term in (3.35): For all $t, \alpha>0$,

$$
\mathrm{e}^{-\alpha t} \sup _{x \in \mathbf{R}^{d}} \mathrm{E}_{x} L_{t}(\phi) \leq \sup _{x \in \mathbf{R}^{d}} \mathrm{E}_{x}\left(\sup _{s>0}\left[\mathrm{e}^{-\alpha s} L_{s}(\phi)\right]\right) .
$$

Therefore, the quantities on the two extreme ends of (3.35) agree up to an explicit multiplicative constant that depends only on $\alpha$ and $t$.

Proof of Lemma 3.6. Because $\phi$ is nonnegative, we have the sure inequality,

$$
\mathrm{e}^{-\alpha s} L_{s}(\phi) \leq \int_{0}^{\infty} \mathrm{e}^{-\alpha r} \phi\left(\bar{X}_{r}\right) \mathrm{d} r, \quad \text { valid for all } s, \alpha>0 .
$$

We take suprema over $s>0$ and then apply expectations $\left[\mathrm{dP}_{x}\right]$ to deduce the first inequality in (3.35). For the second bound, let us note that for all $\alpha, t>0$ and $z \in \mathbf{R}^{d}$,

$$
\left(\bar{R}_{\alpha} \phi\right)(z)=\sum_{n=0}^{\infty} \mathrm{E}_{z} \int_{n t}^{(n+1) t} \mathrm{e}^{-\alpha s} \phi\left(\bar{X}_{s}\right) \mathrm{d} s \leq \sum_{n=0}^{\infty} \mathrm{e}^{-\alpha n t} \mathrm{E}_{z} \int_{0}^{t} \phi\left(\bar{X}_{s+n t}\right) \mathrm{d} s .
$$

This implies the second inequality because

$$
\mathrm{E}_{z} \int_{0}^{t} \phi\left(\bar{X}_{s+n t}\right) \mathrm{d} s=\mathrm{E}_{z} \mathrm{E}_{X_{n t}} \int_{0}^{t} \phi\left(\bar{X}_{s}\right) \mathrm{d} s \leq \sup _{x \in \mathbf{R}^{d}} \mathrm{E}_{x}\left[L_{t}(\phi)\right],
$$

in accordance with the Markov property.

Lemma 3.8. For all $t>0$ and measurable $\phi: \mathbf{R}^{d} \rightarrow \mathbf{R}_{+}$,

$$
\sup _{x \in \mathbf{R}^{d}} \mathrm{E}_{x}\left(\left|L_{t}(\phi)\right|^{2}\right) \leq 2\left(\sup _{z \in \mathbf{R}^{d}} \mathrm{E}_{z}\left[L_{t}(\phi)\right]\right)^{2} .
$$

Proof. We follow some of the ideas in [3] and use subadditivity. Clearly,

$$
\mathrm{E}_{x}\left(\left|L_{t}(\phi)\right|^{2}\right)=2 \int_{0}^{t} \mathrm{~d} u \int_{u}^{t} \mathrm{~d} v \mathrm{E}_{x}\left(\phi\left(\bar{X}_{u}\right) \phi\left(\bar{X}_{v}\right)\right) .
$$

Because the following holds $\mathrm{P}_{x}$-almost surely,

$$
\mathrm{E}_{x}\left(\int_{u}^{t} \phi\left(\bar{X}_{v}\right) \mathrm{d} v \mid \bar{X}_{s} ; s \leq u\right)=\mathrm{E}_{X_{u}} \int_{0}^{t-u} \phi\left(\bar{X}_{s}\right) \mathrm{d} s=\mathrm{E}_{X_{u}}\left[L_{t-u}(\phi)\right],
$$

it follows that

$$
\begin{aligned}
\mathrm{E}_{x}\left(\left|L_{t}(\phi)\right|^{2}\right) & =2 \mathrm{E}_{x} \int_{0}^{t} \phi\left(\bar{X}_{u}\right) \mathrm{E}_{X_{u}}\left[L_{t-u}(\phi)\right] \mathrm{d} u \\
& \leq 2 \mathrm{E}_{x} \int_{0}^{t} \phi\left(\bar{X}_{u}\right) \cdot \sup _{z \in \mathbf{R}^{d}} \mathrm{E}_{z}\left[L_{t-u}(\phi)\right] \mathrm{d} u .
\end{aligned}
$$

We can deduce the lemma because $L_{t-u}(\phi) \leq L_{t}(\phi)$.

The following constitutes the third, and final, technical lemma of this section.

Lemma 3.9. For all $\alpha, t>0$,

$$
\sup _{x \in \mathbf{R}^{d}} \mathrm{P}_{x}\left\{L_{t}(f) \geq \frac{\left(\bar{R}_{\alpha} f\right)(0)}{2 \chi_{\alpha}(t)}\right\} \geq \frac{1}{8}
$$

where $\chi_{\alpha}(t)$ is defined in Lemma 3.6. 
Proof. Recall the Paley-Zygmund inequality [82: If $Z$ is a nonnegative random variable in $L^{2}(\mathrm{P})$ with $\mathrm{E} Z>0$, then

$$
\mathrm{P}\left\{Z \geq \frac{1}{2} \mathrm{E} Z\right\} \geq \frac{(\mathrm{E} Z)^{2}}{4 \mathrm{E}\left(Z^{2}\right)}
$$

We can apply this inequality with $Z:=L_{t}(\phi)$-where $\phi: \mathbf{R}^{d} \rightarrow \mathbf{R}_{+}$is bounded away from zero and infinity - to see that for all $z \in \mathbf{R}^{d}$,

$$
\sup _{x \in \mathbf{R}^{d}} \mathrm{P}_{x}\left\{L_{t}(\phi) \geq \frac{1}{2} \mathrm{E}_{z} L_{t}(\phi)\right\} \geq \frac{\left(\mathrm{E}_{z} L_{t}(\phi)\right)^{2}}{4 \mathrm{E}_{z}\left(\left|L_{t}(\phi)\right|^{2}\right)} .
$$

By selecting $z$ appropriately, we can ensure that

$$
\mathrm{E}_{z} L_{t}(\phi) \geq(1-\epsilon) \cdot \sup _{x \in \mathbf{R}^{d}} \mathrm{E}_{x} L_{t}(\phi),
$$

where $\epsilon \in(0,1)$ is arbitrary but fixed. Let $\epsilon \downarrow 0$ and appeal to the continuity properties of probability measures to deduce that

$$
\sup _{x \in \mathbf{R}^{d}} \mathrm{P}_{x}\left\{L_{t}(\phi) \geq \frac{1}{2} \sup _{z \in \mathbf{R}^{d}} \mathrm{E}_{z} L_{t}(\phi)\right\} \geq \frac{\left(\sup _{z \in \mathbf{R}^{d}} \mathrm{E}_{z} L_{t}(\phi)\right)^{2}}{4 \sup _{x \in \mathbf{R}^{d}} \mathrm{E}_{x}\left(\left|L_{t}(\phi)\right|^{2}\right)} \geq \frac{1}{8} ;
$$

see Lemma 3.8 for the last inequality. A monotone-class argument shows that the preceding holds true for all bounded and measurable functions $\phi \not \equiv 0$. We apply it with $\phi_{N}:=\min (f, N)$ in place of $\phi$, where $N \geq 1$ is fixed. In this way we obtain

$$
\sup _{x \in \mathbf{R}^{d}} \mathrm{P}_{x}\left\{L_{t}(f) \geq \frac{1}{2} \sup _{z \in \mathbf{R}^{d}} \mathrm{E}_{z} L_{t}\left(\phi_{N}\right)\right\} \geq \frac{1}{8} .
$$

This and Lemma 3.6 together tell us that

$$
\sup _{x \in \mathbf{R}^{d}} \mathrm{P}_{x}\left\{L_{t}(f) \geq \frac{\left(\bar{R}_{\alpha} \phi_{N}\right)(0)}{2 \chi_{\alpha}(t)}\right\} \geq \frac{1}{8} .
$$

As $N \uparrow \infty,\left(\bar{R}_{\alpha} \phi_{N}\right)(0) \uparrow\left(\bar{R}_{\alpha} f\right)(0)$, and the lemma follows.

The next result yields a probabilistic characterization of Condition 1.1.

Theorem 3.10. Under Condition 1.1,

$$
\mathrm{P}_{z}\left\{L_{t}(f)<\infty \text { for all } t>0\right\}=1 \quad \text { for all } z \in \mathbf{R}^{d} .
$$

Moreover, in this case, $t \mapsto L_{t}(f)$ grows subexponentially. That is,

$$
\mathrm{P}_{z}\left\{\limsup _{t \rightarrow \infty} \frac{\log L_{t}(f)}{t} \leq 0\right\}=1 \quad \text { for all } z \in \mathbf{R}^{d} .
$$

On the other hand, if Condition 1.1 fails to hold, then

$$
\mathrm{P}_{z}\left\{L_{t}(f)<\infty \text { for some } t>0\right\}=0 \quad \text { for some } z \in \mathbf{R}^{d} .
$$

Remark 3.11. Consider the stochastic heat equation where $\dot{F}$ is space-time white noise. Formally speaking, this means that $f:=\delta_{0}$ is our correlation "function". In this case, one can [again formally] interpret

$$
L_{t}(f)=L_{t}\left(\delta_{0}\right)=\int_{0}^{t} \delta_{0}\left(\bar{X}_{s}\right) \mathrm{d} s
$$

as the local time of the replica process $\bar{X}$ at zero. If we interpret Theorem 3.10 loosely as well, then Theorem 1.3 suggests that (1.1) has a mild solution if and only if $\bar{X}$ has local times. This interpretation is correct, as well as easy to check, 
and leads to deeper connections between SPDEs driven by space-time white noise on one hand and local-time theory on the other hand [42, 46]. In the case of the parabolic Anderson model [that is, (1.1) with $\sigma(u)=$ const $\cdot u$ and $b \equiv 0$ ], Bertini and Cancrini [5] and $\mathrm{Hu}$ and Nualart [57] discuss other closely related connections to local times. In the case where $x$ is a discrete variable [for example, because $\mathcal{L}$ is the generator of a Lévy process on $\mathbf{Z}^{d}$; i.e., the generator of a continuoustime random walk], similar connections were found earlier still; see Carmona and Molchanov 19, for instance.

Proof of Theorem 3.10. If $\left(\bar{R}_{\alpha} f\right)(0)<\infty$ for some $\alpha>0$, then $\left(\bar{R}_{\beta} f\right)(0)<\infty$ for all $\beta>0$ by Theorem 1.2. It follows from the first inequality of Lemma 3.6. and Theorem 1.2 , that

$$
\mathrm{E}_{z}\left[\sup _{t>0}\left(\mathrm{e}^{-\beta t} L_{t}(f)\right)\right] \leq\left(\bar{R}_{\beta} f\right)(0)<\infty \quad\left(\beta>0, z \in \mathbf{R}^{d}\right) .
$$

This implies (3.51); it also implies that

$$
\limsup _{t \rightarrow \infty}\left[\mathrm{e}^{-t \beta} L_{t}(f)\right]<\infty \quad \text { almost surely }\left[\mathrm{P}_{z}\right] \text {. }
$$

We can now deduce (3.52) because $\beta>0$ and $z \in \mathbf{R}^{d}$ are arbitrary.

In order to finish the proof, let us consider the remaining case that $\left(\bar{R}_{\alpha} f\right)(0)=\infty$ for all $\alpha>0$.

According to Lemma 3.9 .

$$
\sup _{x \in \mathbf{R}^{d}} \mathrm{P}_{x}\left\{L_{t}(f)=\infty\right\} \geq \frac{1}{8} \quad(t>0) .
$$

In particular, there exists $z \in \mathbf{R}^{d}$ such that

$$
\mathrm{P}_{z}\left\{L_{t}(f)=\infty \text { for some } t>0\right\} \geq \frac{1}{9} .
$$

Because $t \mapsto L_{t}(f)$ is nondecreasing, the Blumenthal zero-one law applies and implies that $\mathrm{P}_{z}\left\{L_{t}(f)=\infty\right.$ for some $\left.t>0\right\}=1$; this implies the remaining portion of the theorem.

We now have the following consequence of Theorem 3.10. It is particularly useful because its hypotheses are verified by all the examples that we have mentioned in the Introduction.

Corollary 3.12. Suppose $f$ is bounded uniformly on the complement of every open neighborhood of the origin. Then, Condition 1.1 is equivalent to the following: $\mathrm{P}\left\{L_{t}(f)<\infty\right.$ for some $\left.t>0\right\}=1$.

Proof. According to Theorem 3.10 , if Condition 1.1 holds, then

$$
\mathrm{P}_{z}\left\{L_{t}(f)<\infty \text { for some } t>0\right\}=1 \quad \text { for all } z \in \mathbf{R}^{d} .
$$

Set $z:=0$ to obtain half of the corollary.

Conversely, suppose Condition 1.1 fails. According to Theorem 3.10, there exists a point $z \in \mathbf{R}^{d}$ such that

$$
\mathrm{P}_{z}\left\{L_{t}(f)<\infty \text { for some } t>0\right\}=0 .
$$


We need to prove that $z=0$. This holds because if $z$ were not equal to the origin, then $\mathrm{P}_{z}\left\{L_{t}(f)<\infty\right.$ for all $\left.t \in[0, \tau)\right\}=1$, where $\tau$ denotes the first hitting time of the open ball of radius $\|z\| / 2$ around 0 . Indeed,

$$
\sup _{0 \leq t<\tau} L_{t}(f) \leq \tau \cdot \sup _{\|u\| \geq\|z\| / 2} f(u)<\infty,
$$

$\mathrm{P}_{z}$-almost surely. Since the paths of $X$ are right-continuous, $\mathrm{P}_{z}\{\tau>0\}=1$, and hence $(3.60)$ is contradicted.

Condition 1.10 also has a probabilistic interpretation that, in rough terms, $\left(\bar{R}_{\alpha} f\right)(0)<\infty$ if and only if $L_{\infty}(f)<\infty$. The following is a more precise description of this interpretation, as it sorts out the various null sets.

Proposition 3.13. If $\left(\bar{R}_{\alpha} f\right)(0)<\infty$ for some, hence all, $\alpha>0$, then:

$$
\left(\bar{R}_{0} f\right)(0)<\infty \quad \Longrightarrow \quad L_{\infty}(f)<\infty \quad \text { a.s. }
$$

and

$$
\left(\bar{R}_{0} f\right)(0)=\infty \quad \Longrightarrow \quad L_{\infty}(f)=\infty \quad \text { a.s. }
$$

Proof. If $\left(\bar{R}_{0} f\right)(0)<\infty$, then because $\left(\bar{R}_{0} f\right)(0)=\mathrm{E} L_{\infty}(f)<\infty$, it follows that $L_{\infty}(f)$ is finite a.s.

If, on the other hand, $\left(\bar{R}_{0} f\right)(0)=\infty$, then because $\mathrm{E} \int_{0}^{\infty} \mathrm{e}^{-\alpha s} f\left(\bar{X}_{s}\right) \mathrm{d} s=$ $\left(\bar{R}_{\alpha} f\right)(0)$, the Paley-Zygmund inequality (3.45) implies that

$$
\mathrm{P}\left\{\int_{0}^{\infty} \mathrm{e}^{-\alpha s} f\left(\bar{X}_{s}\right) \mathrm{d} s \geq \frac{1}{2}\left(\bar{R}_{\alpha} f\right)(0)\right\} \geq \frac{\left|\left(\bar{R}_{\alpha} f\right)(0)\right|^{2}}{4 \mathrm{E}\left(\left|\int_{0}^{\infty} \mathrm{e}^{-\alpha s} f\left(\bar{X}_{s}\right) \mathrm{d} s\right|^{2}\right)} .
$$

It follows from this and Lemma 3.8 see also the proof of Lemma 3.9 that

$$
\mathrm{P}\left\{\int_{0}^{\infty} f\left(\bar{X}_{s}\right) \mathrm{d} s \geq \frac{1}{2}\left(\bar{R}_{\alpha} f\right)(0)\right\} \geq \frac{\left(\bar{R}_{\alpha} f\right)(0)}{8 \sup _{x \in \mathbf{R}^{d}}\left(\bar{R}_{\alpha} f\right)(x)}=\frac{1}{8},
$$

owing to Theorem 1.2. Let $\alpha \downarrow 0$ to find that

$$
\mathcal{P}:=\mathrm{P}\left\{\int_{0}^{\infty} f\left(\bar{X}_{s}\right) \mathrm{d} s=\infty\right\} \geq \frac{1}{8} .
$$

But according to Theorem 3.10, $\int_{0}^{T} f\left(\bar{X}_{s}\right) \mathrm{d} s<\infty$, for all $T>0$ a.s., because $\left(\bar{R}_{\alpha} f\right)(0)<\infty$ for some [hence all] $\alpha>0$. This implies that

$$
\mathcal{P}=\mathrm{P}\left\{\lim _{T \rightarrow \infty} \int_{T}^{\infty} f\left(\bar{X}_{s}\right) \mathrm{d} s=\infty\right\} .
$$

That is: (i) $\mathcal{P}$ is the probability of a tail event; (ii) $\mathcal{P}$ is strictly positive, in fact $\mathcal{P} \geq 1 / 8$. By the Hewitt-Savage zero-one law [56], $\mathcal{P}=1$.

3.6. A final observation. Let us conclude this section with an observation that will be used later on in Theorem 4.9 in order to produce a stochastic PDE whose random-field solution exists but is densely discontinuous.

Let $\boldsymbol{X}:=\left\{X_{t}\right\}_{t \geq 0}$ denote a Lévy process on $\mathbf{R}^{d}$ with characteristic exponent $\Psi$. Recall that $\boldsymbol{X}$ has a one-potential density $v$ if $v$ is a probability density on $\mathbf{R}^{d}$ that satisfies the following for all Borel-measurable functions $\phi: \mathbf{R}^{d} \rightarrow \mathbf{R}_{+}$:

$$
\mathrm{E}\left[\int_{0}^{\infty} \mathrm{e}^{-s} \phi\left(X_{s}\right) \mathrm{d} s\right]=\int_{\mathbf{R}^{d}} \phi(x) v(x) \mathrm{d} x .
$$


Because $\phi \geq 0$, the preceding expectation commutes with the ds-integral. Recall that $m_{s}$ denotes the law of $X_{s}$, and restrict our attention to only nonnegative $\phi \in \mathcal{S}$. In that case,

$$
\begin{aligned}
\int_{0}^{\infty} \mathrm{e}^{-s} \mathrm{E} \phi\left(X_{s}\right) \mathrm{d} s & =\int_{0}^{\infty} \mathrm{e}^{-s}\left(\int \phi \mathrm{d} m_{s}\right) \mathrm{d} s \\
& =\frac{1}{(2 \pi)^{d}} \int_{0}^{\infty} \mathrm{e}^{-s} \mathrm{~d} s \int_{\mathbf{R}^{d}} \mathrm{~d} \xi \overline{\hat{\phi}(\xi)} \mathrm{e}^{-s \Psi(\xi)} \\
& =\frac{1}{(2 \pi)^{d}} \int_{\mathbf{R}^{d}} \frac{\bar{\phi}(\xi)}{1+\Psi(\xi)} \mathrm{d} \xi
\end{aligned}
$$

We compare this to the right-hand side of (3.68), and then apply Plancherel's theorem to the latter, to deduce the well-known formula:

$$
\hat{v}(\xi)=\frac{1}{1+\Psi(\xi)} \quad\left(\xi \in \mathbf{R}^{d}\right)
$$

If we consider only the case that $\boldsymbol{X}$ is symmetric, then $\hat{v}$ is rendered nonnegative, since $\Psi$ is nonnegative in this case. This observation and the Bochner-Schwartz theorem together imply that $v$ is a correlation function. Because products - and hence integer powers - of correlation functions are themselves correlation functions, Theorem 2.3 yields the following byproduct.

Theorem 3.14. Choose and fix $a>0$ and $b \in \mathbf{R}$. Then, there exists a correlation function $v$ on $\mathbf{R}^{d}$ such that

$$
\hat{v}(\xi) \asymp \frac{1}{\|\xi\|^{a}(\log \|\xi\|)^{b}} \quad \text { for } \xi \in \mathbf{R}^{d} \text { with }\|\xi\|>\mathrm{e} .
$$

\section{THE LINEAR EQUATION}

Before we study the fully nonlinear equation (1.1), we analyse the far simpler linearized form of the same equation $[\sigma \equiv 1, b \equiv 0]$ and show that it has many interesting features of its own. Because the solutions, if any, to the said linear equations can only be Gaussian random fields, we are able to use the theory of Gaussian processes in order to quickly produce some definitive existence and regularity results. This portion of our work should be compared with our earlier joint effort with Eulalia Nualart [46, in which $\dot{F}$ was space-time white noise. Our earlier effort was, in turn, motivated strongly by references [31, 30, 83].

4.1. Existence and uniqueness. The linearized form of (1.1) is the stochastic PDE,

$$
\frac{\partial}{\partial t} u_{t}(x)=\left(\mathcal{L} u_{t}\right)(x)+\dot{F}_{t}(x)
$$

subject to $u_{0}$ being the initial function. As was mentioned in the Introduction, $u_{0}: \mathbf{R}^{d} \rightarrow \mathbf{R}$ is assumed to be a nonrandom bounded and measurable function. One can follow through the theory of Walsh to define the weak solution to (4.1) as the Gaussian random field $u:=\left\{u_{t}(\phi)\right\}_{t>0, \phi \in \mathcal{S}}$, where [we recall] $\mathcal{S}$ denotes the collection of all rapidly-decreasing test functions on $\mathbf{R}^{d}$ and

$$
u_{t}(\phi)=\int_{\mathbf{R}^{d}} u_{0}(x)\left(P_{t}^{*} \phi\right)(x) \mathrm{d} x+\int_{0}^{t} \int_{\mathbf{R}^{d}}\left(p_{t-s} * \phi\right)(y) F(\mathrm{~d} s \mathrm{~d} y) .
$$


The double integral is a Wiener integral and $\left\{P_{t}^{*}\right\}_{t \geq 0}$ is the semigroup associated to the dual process $X_{t}^{*}:=-X_{t}[t \geq 0]$.

There are two main questions that one needs to answer before one proceeds further:

(a) Is $u$ well defined?

(b) What is the largest family of $\phi$ 's for which $u_{t}(\phi)$ is well defined?

An affirmative answer to the first question would imply existence of solutions in the general sense of Walsh 92. Since the analysis of the nonrandom quantity $\int_{\mathbf{R}^{d}} u_{0}(x)\left(P_{t}^{*} \phi\right)(x) \mathrm{d} x$ is standard, we can reduce our problem to the special case that $u_{0} \equiv 0$. In that case, these questions are addressed by the following estimate. Here and throughout, we define

$$
\mathcal{E}_{\lambda}(v):=\frac{1}{2(2 \pi)^{d}} \int_{\mathbf{R}^{d}} \frac{|\hat{v}(\xi)|^{2} \hat{f}(\xi)}{\lambda^{-1}+2 \operatorname{Re} \Psi(\xi)} \mathrm{d} \xi
$$

for all Schwartz distributions $v$ whose Fourier transform is a function.

Next we borrow an observation of [46] in the case of space-time white noise and extend it to the present setting.

Lemma 4.1. The weak solution $u$ to (4.1) with $u_{0} \equiv 0$ exists as a well-defined Gaussian random field parametrized by $t>0$ and $\phi \in \mathcal{S}$. Moreover, for all $t, \lambda>0$ and $\phi \in \mathcal{S}$,

$$
a(t) \mathcal{E}_{\lambda}(\phi) \leq \mathrm{E}\left(\left|u_{t}(\phi)\right|^{2}\right) \leq b(t) \mathcal{E}_{\lambda}(\phi),
$$

where $a(t):=\left(1-\mathrm{e}^{-2 t / \lambda}\right)$ and $b(t):=\mathrm{e}^{2 t / \lambda}$.

In fact, Lemma 4.1 holds under far greater generality than the one presented here. For instance, it holds even when transition functions do not necessarily exist and when the correlation function is a general correlation measure. The less general formulation above suffices for our needs.

Proof. If $\phi \in \mathcal{S}$, then $\hat{p_{t}} \hat{\phi} \in \mathcal{S}$ for all $t \geq 0$. Since the Fourier transform is an isometry on $\mathcal{S}$, this proves that

$$
P_{t}^{*} \phi=p_{t} * \phi \in \mathcal{S} \text { for every } t \geq 0 .
$$

Therefore, in accord with (1.3), the second moment $\mathrm{E}\left(\left|u_{t}(\phi)\right|^{2}\right)$ is equal to

$$
\begin{aligned}
\mathrm{E}\left(\mid \int_{0}^{t} \int_{\mathbf{R}^{d}}\left(P_{t-s}^{*} \phi\right)\right. & \left.\left.(y) F(\mathrm{~d} s \mathrm{~d} y)\right|^{2}\right) \\
& =\frac{1}{(2 \pi)^{d}} \int_{0}^{t} \mathrm{~d} s \int_{\mathbf{R}^{d}} \mathrm{~d} \xi\left|\mathrm{e}^{-(t-s) \Psi(\xi)}\right|^{2} \cdot|\hat{\phi}(\xi)|^{2} \hat{f}(\xi) \\
& =\frac{1}{(2 \pi)^{d}} \int_{0}^{t} \mathrm{~d} s \int \mathrm{d} \xi \mathrm{e}^{-2 s \operatorname{Re}(\xi)} \cdot|\hat{\phi}(\xi)|^{2} \hat{f}(\xi) .
\end{aligned}
$$

The lemma then follows from the preceding and Lemma 3.5 of [46].

There are standard ways to extend the domain of Gaussian random fields. In our case, we proceed as follows: Consider the pseudo-distances $\left\{\rho_{t}\right\}_{t>0}$ defined by

$$
\rho_{t}(\phi, \psi):=\left\{\mathrm{E}\left(\left|u_{t}(\phi)-u_{t}(\psi)\right|^{2}\right)\right\}^{1 / 2} \quad \text { for } \phi, \psi \in \mathcal{S} .
$$


Because $L^{2}(\mathrm{P})$-limits of Gaussian random fields are themselves Gaussian random fields, we deduce the following: Suppose $v$ is a Schwartz distribution such that $\lim _{n \rightarrow \infty} \rho_{t}\left(v, v * \phi_{n}\right)=0$ for all $t>0$, where $\left\{\phi_{n}\right\}_{n=1}^{\infty}$ is the sequence of Gaussian densities, as defined in (3.9) . Then $u_{t}(v)$ is well defined in $L^{2}(\mathrm{P})$, and the totality $\left\{u_{t}(v)\right\}$ of all such random variables forms a Gaussian random field.

We follow [46] and say that (4.1) has a random-field solution if we can obtain $u_{t}\left(\delta_{x}\right)$ in this way for all $x \in \mathbf{R}^{d}$.

Consider the space $\mathcal{H}_{0}$ of all $\phi \in \mathcal{S}$ such that $\mathcal{E}_{1}(\phi)<\infty$ for all $t>0$. Evidently, $\mathcal{H}_{0}$ can be metrized by using the distance

$$
\delta(\phi, \psi):=\sqrt{\mathcal{E}_{1}(\phi-\psi)} \quad \text { for } \psi, \phi \in \mathcal{S} .
$$

Define $\mathcal{H}_{1}$ to be the completion of $\mathcal{H}_{0}$ in the distance $\delta$.

Lemma 4.2. Condition 1.1 holds iff $\delta_{x} \in \mathcal{H}_{1}$ for some, hence all, $x \in \mathbf{R}^{d}$.

Proof. First of all, we recall (1.8) and check that

$$
\mathcal{E}_{\lambda}\left(\delta_{x}\right)=\frac{1}{2(2 \pi)^{d}} \int_{\mathbf{R}^{d}} \frac{\hat{f}(\xi)}{\lambda^{-1}+2 \operatorname{Re} \Psi(\xi)} \mathrm{d} \xi=\frac{1}{2} \Upsilon(1 / \lambda)
$$

In particular, the value of $\mathcal{E}_{\lambda}\left(\delta_{x}\right)$ does not depend on $x \in \mathbf{R}^{d}$. Theorem 1.2 implies that $\mathcal{E}_{\lambda}\left(\delta_{x}\right)$ is finite for some $\lambda>0$ if and only if it is finite for all $\lambda>0$.

Let us first suppose that $\mathcal{E}_{\lambda}\left(\delta_{x}\right)$ is finite. We can note that $\delta_{x} * \phi_{n}=\phi_{n}(\bullet-x) \in \mathcal{S}$, where $\left\{\phi_{n}\right\}_{n=1}^{\infty}$ was defined in (3.9). Therefore, for all $n, m \geq 1$,

$$
\begin{aligned}
\mathcal{E}_{\lambda}\left(\delta_{x}\right. & \left.* \phi_{n}-\delta_{x} * \phi_{m}\right) \\
& =\frac{1}{2(2 \pi)^{d}} \int_{\mathbf{R}^{d}} \frac{\hat{f}(\xi)}{\lambda^{-1}+2 \operatorname{Re} \Psi(\xi)}\left|1-\exp \left\{-\|\xi\|^{2}\left|\frac{1}{2 n}-\frac{1}{2 m}\right|\right\}\right|^{2} \mathrm{~d} \xi
\end{aligned}
$$

Since $\mathcal{E}_{\lambda}\left(\delta_{x}\right)$ is finite, the dominated convergence theorem tells us that the sequence $\left\{\delta_{x} * \phi_{n}\right\}_{n=1}^{\infty}$ is Cauchy in $\mathcal{H}_{0}$. A calculation similar to the preceding shows that the quantity $\mathcal{E}_{\lambda}\left(\delta_{x} * \phi_{n}-\delta_{x}\right)$ converges to zero as $n \rightarrow \infty$. Therefore, $\delta_{x} \in \mathcal{H}_{1}$.

Conversely, if $\delta_{x} \in \mathcal{H}_{1}$, then $\mathcal{E}_{\lambda}\left(\delta_{x} * \phi_{n}-\delta_{x} * \phi_{m}\right) \rightarrow 0$ as $n, m \rightarrow \infty$. We can extract an unbounded subsequence $n_{1} \leq n_{2} \leq \cdots$ of positive integers such that

$$
\mathcal{E}_{\lambda}\left(\delta_{x} * \phi_{n_{j}}-\delta_{x} * \phi_{n_{j+1}}\right) \leq 2^{-j} \text { for all } j \geq 1
$$

It follows from (4.10) that if $k^{-1} \leq\left|n_{j}^{-1}-n_{j+1}^{-1}\right|$, then

$$
\begin{aligned}
\mathcal{E}_{\lambda}\left(\delta_{x}-\delta_{x} * \phi_{k}\right)=\frac{1}{2(2 \pi)^{d}} \int_{\mathbf{R}^{d}} \frac{\hat{f}(\xi)}{\lambda^{-1}+2 \operatorname{Re} \Psi(\xi)}\left|1-\mathrm{e}^{-\|\xi\|^{2} /(2 k)}\right|^{2} \\
\quad \leq \mathcal{E}_{\lambda}\left(\delta_{x} * \phi_{n_{j}}-\delta_{x} * \phi_{n_{j+1}}\right) \leq 2^{-j}
\end{aligned}
$$

Let $k \rightarrow \infty$ and then $j \rightarrow \infty$, in this order, to deduce from the preceding discussion that $\lim _{k \rightarrow \infty} \mathcal{E}_{\lambda}\left(\delta_{x}-\delta_{x} * \phi_{k}\right)=0$. Because $v \mapsto \sqrt{\mathcal{E}_{\lambda}(v)}$ satisfies the triangle 
inequality, it follows from (4.11) that for all $k \geq 2$,

$$
\begin{aligned}
\sqrt{\mathcal{E}_{\lambda}\left(\delta_{x}\right)} & \leq \sqrt{\mathcal{E}_{\lambda}\left(\delta_{x}-\delta_{x} * \phi_{k}\right)}+\sqrt{\mathcal{E}_{\lambda}\left(\delta_{x} * \phi_{k}\right)} \\
& \leq \sqrt{\mathcal{E}_{\lambda}\left(\delta_{x}-\delta_{x} * \phi_{k}\right)}+\sqrt{\mathcal{E}_{\lambda}\left(\delta_{x} * \phi_{k}-\delta_{x} * \phi_{k-1}\right)} \\
& \leq \sqrt{\mathcal{E}_{\lambda}\left(\delta_{x}-\delta_{x} * \phi_{k}\right)}+2^{-(k-1) / 2}+\sqrt{\mathcal{E}_{\lambda}\left(\delta_{x} * \phi_{k-1}\right)} \\
& \vdots \\
& \leq \sqrt{\mathcal{E}_{\lambda}\left(\delta_{x}-\delta_{x} * \phi_{k}\right)}+\sum_{j=1}^{k-1} 2^{-j / 2}+\sqrt{\mathcal{E}_{\lambda}\left(\delta_{x} * \phi_{1}\right)} .
\end{aligned}
$$

We have shown that the first quantity on the right-hand side converges to zero as $k \rightarrow \infty$ and the second term remains bounded. Finally, the third quantity on the right-hand side of the preceding is finite since $\phi_{1} \in \mathcal{S}$. Therefore, it follows that if $\delta_{x} \in \mathcal{H}_{1}$, then $\mathcal{E}_{\lambda}\left(\delta_{x}\right)<\infty$. This concludes our proof.

For more general initial functions $u_{0} \geq 0$, (4.1) has a random-field solution if and only if Condition 1.1 holds and $\left(P_{t} u_{0}\right)(x)<\infty$ for all $t>0$ and $x \in \mathbf{R}^{d}$. Let us conclude this section with a lemma that provides simple conditions that ensure that $\left(P_{t} u_{0}\right)(x)$ is finite for all $t>0$ and $x \in \mathbf{R}^{d}$.

Lemma 4.3. Suppose $\exp (-\operatorname{Re} \Psi) \in L^{t}\left(\mathbf{R}^{d}\right)$ for all $t>0$ and $u_{0} \in L^{\beta}\left(\mathbf{R}^{d}\right)$ for some $\beta \in[1, \infty]$. Then, $\left(P_{t} u_{0}\right)(x)<\infty$ for all $t>0$ and $x \in \mathbf{R}^{d}$. Moreover, $P_{t} u_{0}$ is uniformly bounded and continuous for every fixed $t>0$.

Proof. Since $P_{t}$ is a contraction on $L^{\infty}\left(\mathbf{R}^{d}\right)$, it suffices to consider only the case where $1 \leq \beta<\infty$.

Choose and fix some $t>0$. According to Young's inequality,

$$
\left\|P_{t} u_{0}\right\|_{L^{\infty}\left(\mathbf{R}^{d}\right)}=\left\|\tilde{p}_{t} * u_{0}\right\|_{L^{\infty}\left(\mathbf{R}^{d}\right)} \leq\left\|p_{t}\right\|_{L^{p}\left(\mathbf{R}^{d}\right)} \cdot\left\|u_{0}\right\|_{L^{q}\left(\mathbf{R}^{d}\right)},
$$

where $p^{-1}+q^{-1}=1$. On the other hand, for all $p \in(1, \infty)$,

$$
\left\|p_{t}\right\|_{L^{p}\left(\mathbf{R}^{d}\right)} \leq\left\|p_{t}\right\|_{L^{\infty}\left(\mathbf{R}^{d}\right)},
$$

and this is finite, thanks to Proposition 2.1. Therefore, it remains to prove continuity.

First consider the case that $\beta<\infty$. In that case, we can bound the quantity

$$
\left|\left(P_{t} u_{0}\right)(x)-\left(P_{t} u_{0}\right)\left(x^{\prime}\right)\right|=\left|\left(\tilde{p}_{t} * u_{0}\right)(x)-\left(\tilde{p}_{t} * u_{0}\right)\left(x^{\prime}\right)\right|,
$$

from above, by

$$
\begin{aligned}
\int_{\mathbf{R}^{d}} p_{t}(y) \mid u_{0}(y-x)- & u_{0}\left(y-x^{\prime}\right) \mid \mathrm{d} y \\
& \leq\left(\int_{\mathbf{R}^{d}} p_{t}(y)\left|u_{0}(y-x)-u_{0}\left(y-x^{\prime}\right)\right|^{\beta} \mathrm{d} y\right)^{1 / \beta} \\
& \leq\left\|p_{t}\right\|_{L^{\infty}\left(\mathbf{R}^{d}\right)}^{1 / \beta} \cdot\left\|u_{0}(\bullet-x)-u_{0}\left(\bullet-x^{\prime}\right)\right\|_{L^{\beta}\left(\mathbf{R}^{d}\right)} .
\end{aligned}
$$

It is a classical fact that $u_{0} \in L^{\beta}\left(\mathbf{R}^{d}\right)$ implies that $u_{0}$ is continuous in $L^{\beta}\left(\mathbf{R}^{d}\right)$. Therefore, $P_{t} u_{0}=\tilde{p}_{t} * u_{0}$ is continuous. 
Next let us consider the case where $\beta=\infty$. In that case, we write

$$
\begin{aligned}
\mid\left(\tilde{p}_{t} * u_{0}\right)(x)-\left(\tilde{p}_{t} *\right. & \left.u_{0}\right)\left(x^{\prime}\right) \mid \\
& \leq\left\|u_{0}\right\|_{L^{\infty}\left(\mathbf{R}^{d}\right)} \cdot\left\|p_{t}(\bullet-x)-p_{t}\left(\bullet-x^{\prime}\right)\right\|_{L^{1}\left(\mathbf{R}^{d}\right)},
\end{aligned}
$$

which goes to zero because, once again, $p_{t} \in L^{1}\left(\mathbf{R}^{d}\right)$ implies that $p_{t}$ is continuous in $L^{1}\left(\mathbf{R}^{d}\right)$.

4.2. Spatial regularity: Examples. Define for all $x, y \in \mathbf{R}^{d}$,

$$
d(x, y):=\left(\frac{1}{(2 \pi)^{d}} \int_{\mathbf{R}^{d}} \frac{1-\cos (\xi \cdot(x-y))}{1+2 \operatorname{Re} \Psi(\xi)} \hat{f}(\xi) \mathrm{d} \xi\right)^{1 / 2}
$$

Then, $d$ defines a pseudo-distance on $\mathbf{R}^{d}$. Let $N_{d}$ denote the metric entropy of $[0,1]^{d}$. That is, for all $\epsilon>0, N_{d}(\epsilon)$ denotes the minimum number of radius- $\epsilon d-$ balls required to cover $[0,1]^{d}$. We can combine Lemma 4.1] with theorems of Dudley [72, Theorem 6.1.2, p. 245] and Fernique [72, Theorem 6.2.2, p. 251], together with Belyaev's dichotomy [72, Theorem 5.3.10, p. 213], to deduce the following:

Proposition 4.4. Suppose $\exp (-\operatorname{Re} \Psi) \in L^{t}\left(\mathbf{R}^{d}\right)$, for all $t>0$, and $\Upsilon(1)<\infty$ so that (4.1) has a random-field solution $u$ with $u_{0} \equiv 0$. Then the following are equivalent:

(1) $x \mapsto u_{t}(x)$ has a continuous modification for some $t>0$.

(2) $x \mapsto u_{t}(x)$ has a continuous modification for all $t>0$.

(3) The following metric-entropy condition holds:

$$
\int_{0^{+}}\left(\log N_{d}(\epsilon)\right)^{1 / 2} \mathrm{~d} \epsilon<\infty .
$$

Next we describe a large family of examples for (4.1) that have continuous random-field solutions. Throughout, we write " $h \asymp g$ " in place of " $(h / g)$ is bounded, above and below uniformly, by finite positive constants".

Theorem 4.5. Suppose $f(x)=$ const $/\|x\|^{d-\beta}$ and $\operatorname{Re} \Psi(\xi) \asymp\|\xi\|^{\alpha}(\|\xi\|>1)$ for some $\alpha \in[0,2]$ and $\beta \in(0, d)$. Then (4.1) has a random-field solution if and only if $\alpha+\beta>d$. In this case, (4.20) holds. In fact, we have

$$
d(x, y) \asymp g(\|x-y\|) \quad \text { uniformly when }\|x-y\|<1 / \mathrm{e},
$$

where for all $r \in(0,1 / \mathrm{e})$,

$$
g(r):= \begin{cases}r^{(\alpha+\beta-d) / 2} & \text { if } \alpha+\beta \in(d+1, d+2), \\ r \sqrt{\log (1 / r)} & \text { if } \alpha+\beta=d+2, \\ r & \text { if } \alpha+\beta>d+2 .\end{cases}
$$

Remark 4.6. The condition that $\operatorname{Re} \Psi(\xi) \asymp\|\xi\|^{\alpha}(\|\xi\|>1)$ implies that the upper and lower Blumenthal-Getoor indices of $\Psi$ match and are both equal to $\alpha$. See [12, Theorem 3.2] and 61 for definitions and further details, including the various connections that exist between those indices and the fractal properties of the underlying Lévy process $X$.

Proof of Theorem 4.5. Recall (1.8). In order to prove the existence of random-field solutions, it suffices to show that $\Upsilon(1)<\infty$. We begin by writing

$$
\Upsilon(1)=\frac{1}{(2 \pi)^{d}} \int_{\mathbf{R}^{d}} \frac{\hat{f}(\xi)}{1+2 \operatorname{Re} \Psi(\xi)} \mathrm{d} \xi:=I_{1}+I_{2},
$$


where

$$
\begin{aligned}
& I_{1}:=\frac{1}{(2 \pi)^{d}} \int_{\|\xi\| \leq 1} \frac{\hat{f}(\xi)}{1+2 \operatorname{Re} \Psi(\xi)} \mathrm{d} \xi \quad \text { and } \\
& I_{2}:=\frac{1}{(2 \pi)^{d}} \int_{\|\xi\|>1} \frac{\hat{f}(\xi)}{1+2 \operatorname{Re} \Psi(\xi)} \mathrm{d} \xi
\end{aligned}
$$

It is clear from the hypothesis of the theorem that $I_{1}$ is always finite, because $\beta<d$. We now turn our attention to $I_{2}$ and note that

$$
I_{2} \asymp \int_{\|\xi\|>1} \frac{\mathrm{d} \xi}{\|\xi\|^{\alpha+\beta}} .
$$

Therefore, $I_{2}<\infty$ if and only if $\alpha+\beta>d$. This concludes the first part of the result.

For the second part we assume that

$$
\|x-y\| \leq 1 / \mathrm{e}
$$

We write

$$
|d(x, y)|^{2}:=\frac{1}{(2 \pi)^{d}}\left(J_{1}+J_{2}+J_{3}\right)
$$

where

$$
\begin{aligned}
& J_{1}:=\int_{\|\xi\| \leq 1} \frac{1-\cos (\xi \cdot(x-y))}{1+2 \operatorname{Re} \Psi(\xi)} \hat{f}(\xi) \mathrm{d} \xi \\
& J_{2}:=\int_{\|\xi\|>1 /\|x-y\|} \frac{1-\cos (\xi \cdot(x-y))}{1+2 \operatorname{Re} \Psi(\xi)} \hat{f}(\xi) \mathrm{d} \xi \\
& J_{3}:=\int_{1<\|\xi\| \leq 1 /\|x-y\|} \frac{1-\cos (\xi \cdot(x-y))}{1+2 \operatorname{Re} \Psi(\xi)} \hat{f}(\xi) \mathrm{d} \xi .
\end{aligned}
$$

We can estimate each $J_{j}$ separately.

Because $1-\cos \theta \asymp \theta^{2}$ for $\theta \in(-1,1)$,

$$
\begin{aligned}
J_{1} \asymp\|x-y\|^{2} \cdot \int_{\|\xi\| \leq 1}\|\xi\|^{2} \hat{f}(\xi) \mathrm{d} \xi & \asymp\|x-y\|^{2} \cdot \int_{\|\xi\| \leq 1}\|\xi\|^{2-\beta} \mathrm{d} \xi \\
& \asymp\|x-y\|^{2} \cdot \int_{0}^{1} \frac{\mathrm{d} r}{r^{\beta-d-1}} .
\end{aligned}
$$

Because $\beta<d$, the integral term in the above display is finite, and hence

$$
J_{1} \asymp\|x-y\|^{2} .
$$

We estimate $J_{2}$ similarly:

$$
J_{2} \leq \text { const } \cdot \int_{\|\xi\|>1 /\|x-y\|} \frac{\hat{f}(\xi) \mathrm{d} \xi}{\|\xi\|^{\alpha}} \asymp \int_{1 /\|x-y\|}^{\infty} \frac{\mathrm{d} r}{r^{\alpha+\beta-d+1}} .
$$

The final integral is finite if and only if $\alpha+\beta>d$, and in this case, we have the estimate

$$
0 \leq J_{2} \leq \text { const } \cdot\|x-y\|^{\alpha+\beta-d} .
$$


Finally,

$$
\begin{aligned}
J_{3} & \asymp \int_{1<\|\xi\| \leq 1 /\|x-y\|}\|\xi\|^{2-\alpha-\beta} \cdot\|x-y\|^{2} \mathrm{~d} \xi \\
& \asymp\|x-y\|^{2} \cdot \int_{1}^{1 /\|x-y\|} \frac{\mathrm{d} r}{r^{\alpha+\beta-d-1}} .
\end{aligned}
$$

We evaluate the integrals (4.31) and (4.33) for the different cases of $\alpha+\beta$ to obtain the result.

The following is an immediate consequence of Proposition 4.4 and Theorem 4.5

Corollary 4.7. Every random-field solution u given by Theorem 4.5 has a continuous modification for all $t>0$.

We devote the remainder of this section to a special case of [4.1), namely

$$
\frac{\partial}{\partial t} u_{t}(x)=\left(\Delta u_{t}\right)(x)+\dot{F}_{t}(x) \quad\left(t>0, x \in \mathbf{R}^{3}\right),
$$

where $u_{0} \equiv 0$ and the Laplacian acts on the $x$ variable only. The noise $F$ is a centered Gaussian noise, as before, that is white in time and homogeneous in space with a correlation function $f$ that satisfies the following for a fixed $q \in \mathbf{R}$ :

$$
\hat{f}(\xi) \asymp \frac{1}{\|\xi\|(\log \|\xi\|)^{q}} \quad \text { for } \xi \in \mathbf{R}^{3} \text { with }\|\xi\|>\text { e. }
$$

According to Theorem 3.14, such correlation functions exist.

The following lemma will be useful for the proof of the main result of this section.

Lemma 4.8. If $g: \mathbf{R}^{3} \mapsto \mathbf{R}_{+}$is a Borel-measurable radial function, then

$$
\int_{\|x\|>1 /\|y\|}(1-\cos (x \cdot y)) g(x) \mathrm{d} x \geq \text { const } \cdot \int_{\|x\|>1 /\|y\|} g(x) \mathrm{d} x,
$$

uniformly for all $y \in \mathbf{R}^{3} \backslash\{0\}$.

Proof. Clearly,

$$
\begin{aligned}
\int_{\|x\|>1 /\|y\|}(1-\cos (x \cdot y)) & g(x) \mathrm{d} x \\
= & \int_{1 /\|y\|}^{\infty} r^{2} R(r) \mathrm{d} r \int_{\mathbf{S}^{2}} \mathrm{~d} \theta(1-\cos (y \cdot r \theta)),
\end{aligned}
$$

where $R$ is the function on $\mathbf{R}_{+}$defined by $R(\|x\|):=g(x)$ for all $x \in \mathbf{R}^{3}$. But for all $r>0$, the $\mathrm{d} \theta$-integral can be computed as

$$
\int_{\mathbf{S}^{2}}(1-\cos (y \cdot r \theta)) \mathrm{d} \theta=\text { const } \cdot\left(1-\frac{\sin (r\|y\|)}{r\|y\|}\right),
$$

and this is bounded below uniformly, as long as $r>1 /\|y\|$. We combine the preceding two displays to obtain the result.

The following is the main result concerning (4.34).

Theorem 4.9. Consider the stochastic heat equation (4.34) in $\mathbf{R}^{3}$, where the correlation function $f$ of the noise satisfies (4.35) for a given fixed value $q \in \mathbf{R}$. Then:

(a) (4.34) has a random-field solution $u$ iff $q>1$;

(b) $x \mapsto u_{t}(x)$ has a continuous modification for all $t>0$ iff $q>2$. 
Remark 4.10. Theorem 4.9 and general facts about stationary Gaussian processes [see Belyaev's dichotomy [72, Theorem 5.3.10, p. 213], for instance] together prove that when $q \in(1,2]$, the stochastic heat equation (4.35) has a random-field solution $u$ that almost surely has infinite oscillations in every open space-time set. This example was mentioned at the end of the Introduction.

Proof. In order to show the existence of a random-field solution it suffices to show that $\Upsilon(1)<\infty$ if and only if $q>1$. Because $\Psi(\xi)=\|\xi\|^{2}$, we may write $\Upsilon(1)$ as follows:

$$
\Upsilon(1)=\frac{1}{8 \pi^{3}} \int_{\mathbf{R}^{3}} \frac{\hat{f}(\xi)}{1+2\|\xi\|^{2}} \mathrm{~d} \xi:=\frac{I_{1}+I_{2}}{8 \pi^{3}},
$$

where

$$
I_{1}:=\int_{\|\xi\|<\mathrm{e}} \frac{\hat{f}(\xi)}{1+2\|\xi\|^{2}} \mathrm{~d} \xi \quad \text { and } \quad I_{2}:=\int_{\|\xi\|>\mathrm{e}} \frac{\hat{f}(\xi)}{1+2\|\xi\|^{2}} \mathrm{~d} \xi .
$$

Direct inspection reveals that

$$
I_{1} \asymp \int_{0}^{\mathrm{e}} \frac{r}{(\log r)^{q}} \mathrm{~d} r \quad \text { and } \quad I_{2} \asymp \int_{\mathrm{e}}^{\infty} \frac{1}{r(\log r)^{q}} \mathrm{~d} r .
$$

It follows readily from this that $\Upsilon(1)<1$ if and only if $q>1$.

We now turn our attention to the second part of the proof. Throughout, we assume that $\|x-y\| \leq 1$ /e and consider the following integral:

$$
|d(x, y)|^{2}=\frac{1}{8 \pi^{3}} \int_{\mathbf{R}^{3}} \frac{1-\cos (\xi \cdot(x-y))}{1+2\|\xi\|^{2}} \hat{f}(\xi) \mathrm{d} \xi:=\frac{J_{1}+J_{2}+J_{3}}{8 \pi^{3}},
$$

where

$$
\begin{aligned}
J_{1} & :=\int_{\|\xi\| \leq \mathrm{e}} \frac{1-\cos (\xi \cdot(x-y))}{1+2\|\xi\|^{2}} \hat{f}(\xi) \mathrm{d} \xi \\
J_{2} & :=\int_{\|\xi\|>1 /\|x-y\|} \frac{1-\cos (\xi \cdot(x-y))}{1+2\|\xi\|^{2}} \hat{f}(\xi) \mathrm{d} \xi \\
J_{3} & :=\int_{\mathrm{e}<\|\xi\| \leq 1 /\|x-y\|} \frac{1-\cos (\xi \cdot(x-y))}{1+2\|\xi\|^{2}} \hat{f}(\xi) \mathrm{d} \xi .
\end{aligned}
$$

We estimate each of the integrals separately. The first term can be dealt with easily, and we obtain the following, using similar computations to those in the proof of Theorem 4.5 .

$$
J_{1} \asymp\|x-y\|^{2} .
$$

The estimation of the second term requires a little bit more work, viz.,

$$
\begin{aligned}
J_{2} & \leq \frac{1}{2} \int_{\|\xi\|>1 /\|x-y\|} \frac{1-\cos (\xi \cdot(x-y))}{\|\xi\|^{2}} \hat{f}(\xi) \mathrm{d} \xi \\
& \leq \text { const } \cdot \int_{\|\xi\|>1 /\|x-y\|} \frac{\hat{f}(\xi)}{\|\xi\|^{2}} \mathrm{~d} \xi \leq \mathrm{const} \cdot \int_{1 /\|x-y\|}^{\infty} \frac{1}{r(\log r)^{q}} \mathrm{~d} r \\
& =\text { const } \cdot\left(\log \frac{1}{\|x-y\|}\right)^{-q+1} .
\end{aligned}
$$


Finally, we consider the final term $J_{3}$ :

$$
\begin{aligned}
J_{3} \asymp\|x-y\|^{2} \int_{\mathrm{e}<\|\xi\| \leq 1 /\|x-y\|} \hat{f}(\xi) \mathrm{d} \xi & \asymp\|x-y\|^{2} \int_{\mathrm{e}}^{1 /\|x-y\|} \frac{r}{(\log r)^{q}} \mathrm{~d} r \\
& \leq \text { const } \cdot\left(\log \frac{1}{\|x-y\|}\right)^{-q} .
\end{aligned}
$$

Upon combining the above estimates we obtain the bound

$$
|d(x, y)|^{2} \leq \mathrm{const} \cdot\left(\log \frac{1}{\|x-y\|}\right)^{-q+1} .
$$

Next, we compute a similar lower bound for $|d(x, y)|^{2}$. Since the integrands are nonnegative throughout, we may consider only $J_{2}$. In that case, Lemma 4.8 yields the following:

$$
J_{2} \geq \text { const } \cdot \int_{\|\xi\| \geq 1 /\|x-y\|} \frac{\hat{f}(\xi)}{\|\xi\|^{2}} \mathrm{~d} \xi \geq \text { const } \cdot\left(\log \frac{1}{\|x-y\|}\right)^{-q+1} .
$$

The preceding discussion implies that

$$
d(x, y) \asymp|\log (\|x-y\|)|^{(1-q) / 2},
$$

uniformly, as long as $\|x-y\|<1 /$ e. From this, we obtain

$$
\log N_{d}(\epsilon) \asymp \epsilon^{2 /(1-q)},
$$

valid for $0<\epsilon<1$ /e. In particular, the metric-entropy condition (4.20) applies if and only if $q>2$. Since the other conditions of Proposition 4.4 hold [for elementary reasons], the second part of the theorem follows from Proposition 4.4 .

\section{THE NONLINEAR EQUATION}

The primary goal of this section is to study the fully-nonlinear stochastic heat equation (1.1) as described in the Introduction.

In the first part, we derive a series of a priori estimates that will ultimately lead to the proof of Theorem [1.3. The latter theorem shows that the finite-potential Condition 1.1 is sufficient for the existence of a mild solution to the stochastic heat equation. As a byproduct, that theorem also yields a temporal growth rate for the solution. This means that under some natural conditions on the multiplicative nonlinearity $\sigma$, the mild solution will not be intermittent.

The second part is devoted to the proofs of Theorems 1.8 and 1.11, and thereby establishing the fact that, in contrast to the preceding discussion, if "there is enough symmetry and nonlinearity", then the mild solution to the stochastic heat equation is weakly intermittent.

In the third and final part, we give a partial answer to a deep question of Professor David Nualart who asked about the "effect of drift" on the intermittence of the solution. In particular, we show that if the drift is exactly linear-which corresponds to a massive and/or dissipative version of (1.1) - then there is frequently an explicit phase transition which describes the amount of drift needed in order to offset the intermittent multiplicative effect of the underlying noise. 
5.1. Proof of Theorem 1.3, We rewrite (1.1), in mild form, as follows:

$$
\begin{aligned}
u_{t}(x)=\left(P_{t} u_{0}\right)(x)+\int_{0}^{t} \mathrm{~d} s \int_{\mathbf{R}^{d}} \mathrm{~d} y p_{t-s}(y-x) b\left(u_{s}(y)\right) \\
\quad+\int_{0}^{t} \int_{\mathbf{R}^{d}} p_{t-s}(y-x) \sigma\left(u_{s}(y)\right) F(\mathrm{~d} s \mathrm{~d} y) .
\end{aligned}
$$

As is customary (see [30, for instance) we seek to find a mild solution that satisfies the following integrability condition:

$$
\sup _{t \in[0, T]} \sup _{x \in \mathbf{R}^{d}} \mathrm{E}\left(\left|u_{t}(x)\right|^{2}\right)<\infty \quad \text { for all } T>0 .
$$

In order to prove Theorem 1.3 we apply a familiar fixed-point argument, though the details of this argument are not entirely standard.

Let $\mathcal{F}:=\left\{\mathcal{F}_{t}\right\}_{t>0}$ denote the right-continuous complete filtration generated by the noise $F$. Specifically, for every positive $t$, we define $\mathcal{F}_{t}^{0}$ to be the $\sigma$-algebra generated by random variables of the form of the Wiener integral

$$
\int_{[0, t] \times \mathbf{R}^{d}} \phi \mathrm{d} F:=\int_{[0, t] \times \mathbf{R}^{d}} \phi_{s}(x) F(\mathrm{~d} s \mathrm{~d} x),
$$

as $\phi$ ranges over $L^{2}\left([0, t] \times \mathbf{R}^{d}\right)$. Define $\mathcal{F}_{t}^{1}$ to be the P-completion of $\mathcal{F}_{t}^{0}$, and finally define $\mathcal{F}_{t}:=\bigcap_{s>t} \mathcal{F}_{s}^{1}$ as the right-continuous extension.

We recall from Walsh 92 , that a random field $\left\{v_{t}(x)\right\}_{t>0, x \in \mathbf{R}^{d}}$ is predictable if it can be realized as an $L^{2}(\mathrm{P})$-limit of finite linear combination of random fields of the type

$$
z_{t}(x)(\omega):=X(\omega) \mathbf{1}_{(a, b] \times A}(t, x) \quad \text { for } t>0, x \in \mathbf{R}^{d}, \text { and } \omega \in \Omega,
$$

where $0<a<b<\infty, A \subseteq \mathbf{R}^{d}$ is compact, and $X$ is an $\mathcal{F}_{t}$-measurable and bounded random variable. [We are using the standard " $(\Omega, \mathcal{F}, \mathrm{P})$ " notation of probability for the underlying probability space, of course.]

Define for all predictable random fields $v$,

$$
(\mathcal{A} v)_{t}(x):=\int_{[0, t] \times \mathbf{R}^{d}} p_{t-s}(y-x) \sigma\left(v_{s}(y)\right) F(\mathrm{~d} s \mathrm{~d} y)
$$

and

$$
(\mathcal{B} v)_{t}(x):=\int_{0}^{t} \mathrm{~d} s \int_{\mathbf{R}^{d}} \mathrm{~d} y p_{t-s}(y-x) b\left(v_{s}(y)\right),
$$

provided that the integrals exist. The first integral must exist in the sense of Walsh 92, and the second in the sense of Lebesgue.

Define for all $\beta, p>0$ and all predictable random fields $v$,

$$
\|v\|_{\beta, p}:=\sup _{t>0} \sup _{x \in \mathbf{R}^{d}}\left[\mathrm{e}^{-\beta t} \mathrm{E}\left(\left|v_{t}(x)\right|^{p}\right)\right]^{1 / p} .
$$

It is easy to see that the preceding defines a [pseudo-] norm on random fields, for every fixed choice of $\beta, p>0$. In fact, these are one among many possible infinitedimensional $L^{p}$-norms. The corresponding $L^{p}$-type space is denoted by $\boldsymbol{B}_{\beta, p}$. We make the following definition which will be in force throughout the rest of the paper.

Definition 5.1. Let $\boldsymbol{B}_{\beta, p}$ denote the collection of all [equivalence classes of modifications of] predictable random fields $X:=\left\{X_{t}(x)\right\}_{t \geq 0, x \in \mathbf{R}^{d}}$ such that $\|X\|_{\beta, p}<\infty$. 
One can easily check that $\|\cdot\|_{\beta, p}$ defines a pseudo-norm on $\boldsymbol{B}_{\beta, p}$. Moreover, if we identify $X \in \boldsymbol{B}_{\beta, p}$ with $Y \in \boldsymbol{B}_{\beta, p}$ when $\|X-Y\|_{\beta, p}=0$, then [the resulting collection of equivalence classes in] $\boldsymbol{B}_{\beta, p}$ becomes a Banach space. Because $\| X-$ $Y \|_{\beta, p}=0$ if and only if $X$ and $Y$ are modifications of one another, it follows thatafter the usual identification of a process with its modifications $-\boldsymbol{B}_{\beta, p}$ is a Banach space of [equivalence classes of] functions with finite $\|\cdot\|_{\beta, p}$ norm.

Our next two lemmas contain a priori estimates on Walsh-type stochastic integrals, as well as certain Lebesgue integrals. Among other things, these lemmas show that $\mathcal{B}$ and $\mathcal{A}$ are bounded linear maps from predictable processes to predictable processes. These lemmas are strongly motivated by the theory of optimal regularity for parabolic equations, as is our entire approach to the proof of Theorem 1.3, see Lunardi 68. We follow the main idea of optimal regularity and aim to find a good function space such that if $u_{0}$ resides in that function space, then $u_{t}$ has to live in the same function space for all $t$. As we shall soon see, the previously-defined Banach spaces $\left\{\boldsymbol{B}_{\beta, p}\right\}_{\beta, p>0}$ form excellent candidates for those function spaces. In a rather different context, this general idea also appears in Dalang and Mueller [35. Those authors show that $L^{2}\left(\mathbf{R}^{d}\right)$ is also a good candidate for such a function space provided that $\sigma(0)=0$.

Here and throughout, we will use the following notation on Lipschitz functions.

Convention 5.2. If $g: \mathbf{R}^{d} \rightarrow \mathbf{R}$ is Lipschitz continuous, then we can find finite constants $\mathrm{C}_{g}$ and $\mathrm{D}_{g}$ such that

$$
|g(x)| \leq \mathrm{C}_{g}+\mathrm{D}_{g}|x| \text { for all } x \in \mathbf{R}^{d} .
$$

To be concrete, we choose $\mathrm{C}_{g}:=|g(0)|$ and $\mathrm{D}_{g}:=\operatorname{Lip}_{g}$ to be concrete.

As mentioned above, the next two results describe a priori estimates for the Walsh-integral-processes $\mathcal{B} v$ and $\mathcal{A} v$ when $v$ is a nice predictable random field. Together, they imply that the random linear operators $\mathcal{A}$ and $\mathcal{B}$ map each and every $\boldsymbol{B}_{\beta, p}$ into itself boundedly and continuously. The respective operator norms are both described in terms of a replica potential of the correlation function $f$.

Lemma 5.3. For all integers $p \geq 2$, real numbers $\beta>0$, and predictable random fields $v$ and $w$,

$$
\|\mathcal{B} v\|_{\beta, p} \leq \frac{p}{\beta}\left(\frac{\mathrm{C}_{b}}{\mathrm{e}}+\mathrm{D}_{b}\|v\|_{\beta, p}\right)
$$

and

$$
\|\mathcal{B} v-\mathcal{B} w\|_{\beta, p} \leq \frac{p \operatorname{Lip}_{b}}{\beta}\|v-w\|_{\beta, p}
$$

Proof. On one hand, the triangle inequality implies that $\mathrm{E}\left(\left|(\mathcal{B} u)_{t}(x)\right|^{p}\right)$ is bounded above by the following quantity:

$$
\int_{0}^{t} \mathrm{~d} s_{1} \int_{\mathbf{R}^{d}} \mathrm{~d} y_{1} \cdots \int_{0}^{t} \mathrm{~d} s_{p} \int_{\mathbf{R}^{d}} \mathrm{~d} y_{p} \prod_{k=1}^{p} p_{t-s_{k}}\left(t_{k}-x\right) \cdot \mathrm{E}\left(\prod_{j=1}^{p}\left|b\left(v_{s_{j}}\left(y_{j}\right)\right)\right|\right) .
$$

On the other hand, the generalized Hölder inequality tells us that

$$
\mathrm{E}\left(\prod_{j=1}^{p}\left|b\left(v_{s_{j}}\left(y_{j}\right)\right)\right|\right) \leq \prod_{j=1}^{p}\left\|b\left(v_{s_{j}}\left(y_{j}\right)\right)\right\|_{p} .
$$


Therefore, we can conclude that

$$
\mathrm{E}\left(\left|(\mathcal{B} v)_{t}(x)\right|^{p}\right) \leq\left(\mathrm{C}_{b} t+\mathrm{D}_{b} \int_{0}^{t} \mathrm{~d} s \int_{\mathbf{R}^{d}} \mathrm{~d} y p_{t-s}(y-x)\left\|v_{s}(y)\right\|_{p}\right)^{p} .
$$

We multiply the preceding by $\exp (-\beta t)$ and take the the $(1 / p)$-th root to find that

$$
\begin{aligned}
& {\left[\mathrm{e}^{-\beta t} \mathrm{E}\left(\left|(\mathcal{B} v)_{t}(x)\right|^{p}\right)\right]^{1 / p}} \\
& \leq \mathrm{C}_{b} t \mathrm{e}^{-\beta t / p}+\mathrm{D}_{b} \int_{0}^{\infty} \mathrm{d} s \int_{\mathbf{R}^{d}} \mathrm{~d} y \mathrm{e}^{-\beta(t-s) / p} p_{t-s}(y-x) \mathrm{e}^{-\beta s / p}\left\|v_{s}(y)\right\|_{p} \\
& \leq \mathrm{C}_{b} t \mathrm{e}^{-\beta t / p}+\frac{p \mathrm{D}_{b}}{\beta}\|v\|_{\beta, p} .
\end{aligned}
$$

The first display of the lemma follows because $t \exp (-\beta t / p) \leq p /(\mathrm{e} \beta)$ for all $t>0$. In order to obtain the second display we note that

$$
\left|(\mathcal{B} v)_{t}(x)-(\mathcal{B} w)_{t}(x)\right| \leq \operatorname{Lip}_{b} \cdot\left(\mathcal{B}_{1}(|v-w|)\right)_{t}(x),
$$

where $\mathcal{B}_{1}$ is defined exactly as $\mathcal{B}$ was, but with $b(x)$ replaced by $b_{1}(x)=x$. Because we may choose $\mathrm{C}_{b_{1}}=0$ and $\mathrm{D}_{b_{1}}=1$, the second assertion of the lemma follows from the first.

Lemma 5.4. For all even integers $p \geq 2$, real numbers $\beta>0$, and predictable random fields $v$ and $w$,

$$
\|\mathcal{A} v\|_{\beta, p} \leq z_{p}\left(\mathrm{C}_{\sigma}+\mathrm{D}_{\sigma}\|v\|_{\beta, p}\right) \sqrt{\left(\bar{R}_{2 \beta / p} f\right)(0)}
$$

and

$$
\|\mathcal{A} v-\mathcal{A} w\|_{\beta, p} \leq z_{p} \operatorname{Lip}_{\sigma}\|v-w\|_{\beta, p} \sqrt{\left(\bar{R}_{2 \beta / p} f\right)(0)} .
$$

Proof. According to Davis's formulation [38] of the Burkholder-Davis-Gundy inequality, $\mathrm{E}\left(\left|(\mathcal{A} v)_{t}(x)\right|^{p}\right)$ is bounded above by $z_{p}^{p}$ times the expectation of

$$
\left|\int_{0}^{t} \mathrm{~d} s \int_{\mathbf{R}^{d}} \mathrm{~d} y \int_{\mathbf{R}^{d}} \mathrm{~d} z V_{s}(y, z) p_{t-s}(y-x) p_{t-s}(z-x) f(z-y)\right|^{p / 2},
$$

where

$$
V_{s}(y, z):=\sigma\left(v_{s}(y)\right) \sigma\left(v_{s}(z)\right) .
$$

By the generalized Hölder inequality,

$$
\mathrm{E}\left(\prod_{j=1}^{p / 2} V_{s_{j}}\left(y_{j}, z_{j}\right)\right) \leq \prod_{j=1}^{p / 2}\left\|\sigma\left(v_{s_{j}}\left(y_{j}\right)\right)\right\|_{p}\left\|\sigma\left(v_{s_{j}}\left(z_{j}\right)\right)\right\|_{p} .
$$

Consequently, $\mathrm{E}\left(\left|(\mathcal{A} v)_{t}(x)\right|^{p}\right)$ is bounded above by $z_{p}^{p}$ times

$$
\left(\int_{0}^{t} \mathrm{~d} s \int_{\mathbf{R}^{d}} \mathrm{~d} y \int_{\mathbf{R}^{d}} \mathrm{~d} z V_{s}^{\prime}(y, z) p_{t-s}(y-x) p_{t-s}(z-x) f(z-y)\right)^{p / 2},
$$

where

$$
V_{s}^{\prime}(y, z):=\left\|\sigma\left(v_{s}(y)\right)\right\|_{p}\left\|\sigma\left(v_{s}(z)\right)\right\|_{p} .
$$


We can note that for all $s>0$ and $y \in \mathbf{R}^{d}$,

$$
\begin{aligned}
\left\|\sigma\left(v_{s}(y)\right)\right\|_{p} \leq \mathrm{C}_{\sigma}+\mathrm{D}_{\sigma}\left\|v_{s}(y)\right\|_{p} & \leq \mathrm{C}_{\sigma}+\mathrm{D}_{\sigma} \mathrm{e}^{\beta s / p}\|v\|_{\beta, p} \\
& \leq \mathrm{e}^{\beta s / p}\left(\mathrm{C}_{\sigma}+\mathrm{D}_{\sigma}\|v\|_{\beta, p}\right) .
\end{aligned}
$$

Therefore, a line or two of computations yield

$$
\mathrm{E}\left(\left|(\mathcal{A} v)_{t}(x)\right|^{p}\right) \leq \mathrm{e}^{\beta t} z_{p}^{p}\left(\mathrm{C}_{\sigma}+\mathrm{D}_{\sigma}\|v\|_{\beta, p}\right)^{p}\left(\int_{0}^{\infty} \mathrm{e}^{-2 \beta s / p} \mathcal{H}_{s} \mathrm{~d} s\right)^{p / 2},
$$

where

$$
\mathcal{H}_{s}:=\int_{\mathbf{R}^{d}} \mathrm{~d} a \int_{\mathbf{R}^{d}} \mathrm{~d} b f(a-b) p_{s}(a) p_{s}(b)=\left(P_{s}^{*} P_{s} f\right)(0)=\left(\bar{P}_{s} f\right)(0)
$$

Hence,

$$
\mathrm{E}\left(\left|(\mathcal{A} v)_{t}(x)\right|^{p}\right) \leq \mathrm{e}^{\beta t} z_{p}^{p}\left(\mathrm{C}_{\sigma}+\mathrm{D}_{\sigma}\|v\|_{\beta, p}\right)^{p}\left(\left(\bar{R}_{2 \beta / p} f\right)(0)\right)^{p / 2} .
$$

The first assertion of the lemma follows immediately from this.

In order to deduce the second assertion we note that

$$
\|\mathcal{A} v-\mathcal{A} w\|_{\beta, p} \leq \operatorname{Lip}_{\sigma} \cdot \mathcal{A}_{1}(|v-w|),
$$

where $\mathcal{A}_{1}$ is the same as $\mathcal{A}$, but with $\sigma(x)$ replaced by $\sigma_{1}(x)=x$. Therefore, the first assertion of the lemma implies the second. This completes the proof.

We are ready to begin a more-or-less standard iterative construction that is used to prove Theorem 1.3 .

Let

$$
u_{t}^{0}(x):=u_{0}(x),
$$

and define iteratively: For all $n \geq 0$,

$$
u_{t}^{n+1}(x)=\left(P_{t} u_{0}\right)(x)+\left(\mathcal{B} u^{n}\right)_{t}(x)+\left(\mathcal{A} u^{n}\right)_{t}(x) .
$$

Dalang's theory 30 tells us that the solution $u$ to (1.1) can be obtained pointwise as $\lim _{n \rightarrow \infty} u_{t}^{n}(x)$, where the limit takes place in $L^{2}(\mathrm{P})$ and holds uniformly in $x \in \mathbf{R}^{d}$ and locally uniformly for $t>0$. We need to strengthen the sense in which that limit holds. The following is a key step toward reaching that goal.

Lemma 5.5. Choose and fix $\beta>0$ and an even integer $p \geq 2$. If

$$
\frac{p \mathrm{D}_{b}}{\beta}+z_{p} \mathrm{D}_{\sigma} \sqrt{\left(\bar{R}_{2 \beta / p} f\right)(0)}<1,
$$

then $\sup _{n \geq 0}\left\|u^{n}\right\|_{\beta, p}<\infty$.

Proof. Because $P_{t} u_{0}$ is bounded, uniformly in modulus, by $\sup _{x \in \mathbf{R}^{d}}\left|u_{0}(x)\right|$, the triangle inequality implies that

$$
\left\|u^{n+1}\right\|_{\beta, p} \leq \sup _{x \in \mathbf{R}^{d}}\left|u_{0}(x)\right|+\left\|\mathcal{B} u^{n}\right\|_{\beta, p}+\left\|\mathcal{A} u^{n}\right\|_{\beta, p} .
$$

Lemmas 5.3 and 5.4 , and a few lines of direct computation, together imply that

$$
\left\|u^{n+1}\right\|_{\beta, p} \leq A+B\left\|u^{n}\right\|_{\beta, p}
$$

where

$$
A:=\sup _{x \in \mathbf{R}^{d}}\left|u_{0}(x)\right|+\frac{p \mathrm{C}_{b}}{\beta \mathrm{e}}+z_{p} \mathrm{C}_{\sigma} \sqrt{\left(\bar{R}_{2 \beta / p} f\right)(0)}
$$


and

$$
B:=\frac{p \mathrm{D}_{b}}{\beta}+z_{p} \mathrm{D}_{\sigma} \sqrt{\left(\bar{R}_{2 \beta / p} f\right)(0)}
$$

Iteration yields the bound

$$
\left\|u^{n+1}\right\|_{\beta, p} \leq A\left(1+B+\cdots+B^{n-1}+B^{n} \sup _{x \in \mathbf{R}^{d}}\left|u_{0}(x)\right|\right) .
$$

Consequently, if $B<1$, then

$$
\sup _{k \geq 1}\left\|u^{k}\right\|_{\beta, p} \leq \frac{A}{1-B} .
$$

Since $\left\|u^{0}\right\|_{\beta, p} \leq \sup _{x \in \mathbf{R}^{d}}\left|u_{0}(x)\right|<\infty$, the lemma follows.

We now have all the technical estimates for the proof of Theorem 1.3 .

5.2. Proof of Theorem 1.3. Without loss of generality, we can find $\beta>0$ such that $Q(p, \beta)<1$, otherwise there is nothing to prove. Choose and fix such a $\beta$.

Thanks to Lemma 5.5, every $u^{n}$ is well defined and $\left\|u^{n}\right\|_{\beta, p}$ is finite, uniformly in $n$. In particular, $u^{n} \in \boldsymbol{B}_{\beta, p}$. Next we apply Lemmas 5.3 and 5.4-with $\mathrm{C}_{g}:=|g(0)|$ and $\mathrm{D}_{g}:=\operatorname{Lip}_{g}$ - to find that

$$
\begin{aligned}
\left\|u^{n+1}-u^{n}\right\|_{\beta, p} & \leq\left\|\mathcal{B} u^{n}-\mathcal{B} u^{n-1}\right\|_{\beta, p}+\left\|\mathcal{A} u^{n}-\mathcal{A} u^{n-1}\right\|_{\beta, p} \\
& \leq\left\|u^{n}-u^{n-1}\right\|_{\beta, p} \cdot Q(p, \beta) .
\end{aligned}
$$

Because $Q(p, \beta)<1$, the preceding implies that

$$
\sum_{n=1}^{\infty}\left\|u^{n+1}-u^{n}\right\|_{\beta, p} \leq \mathrm{const} \cdot \sum_{n=1}^{\infty}\{Q(p, \beta)\}^{n}<\infty .
$$

Therefore, we can find a predictable random field $u^{\infty} \in \boldsymbol{B}_{\beta, p}$ such that $\lim _{n \rightarrow \infty} u^{n}=$ $u^{\infty}$ in $\boldsymbol{B}_{\beta, p}$. Our arguments can be adjusted to also show that $\lim _{n \rightarrow \infty} \mathcal{B} u^{n}=\mathcal{B} u^{\infty}$ and $\lim _{n \rightarrow \infty} \mathcal{A} u^{n}=\mathcal{A} u^{\infty}$, with both limits taking place in $\boldsymbol{B}_{\beta, p}$. This discussion proves that $u^{\infty}$ is another solution to (5.1). Because of the theory of [30], $u$ is the almost-surely unique solution to (5.1). Therefore, $u^{\infty}$ is equal to $u$ up to evanescence, and hence $u \in \boldsymbol{B}_{\beta, p}$ for all $\beta>0$ such that $Q(p, \beta)<1$. This proves the theorem.

5.3. Intermittency. We now prove Theorems 1.8 and 1.11 They state, in one form or another, that the solution to the stochastic heat equation can be weakly intermittent in the presence of enough symmetry and nonlinearity. The basic idea is to follow, and adapt, our earlier work on space-time white noise [45] and apply classical renewal-theoretic ideas [21]. However, because of the spatial correlation of the noise, that adaptation quickly meets obstacles that do not exist when considering space-time white noise.

It might help to recall the definition (1.8) of the function $\Upsilon$, and the relation [Theorem 1.2 between the positive number $\Upsilon(\beta)$ and the maximum value of the replica $\beta$-potential of the correlation function $f$, as well as the positive number $\left(\bar{R}_{\beta} f\right)(0)$ of that replica potential at the origin. 
Proof of Theorem 1.8. We can assume, without loss of generality, that there exists $\beta>0$ such that

$$
\Upsilon(\beta) \geq 2 / \mathrm{L}_{\sigma}^{2},
$$

for there is nothing left to prove otherwise. Now consider any such $\beta$.

Since $b(x)=0, u_{0} \geq \eta$, and $\sigma(u) \geq \mathrm{L}_{\sigma}|u|$, we can apply (5.1) to deduce that for all $x, y \in \mathbf{R}^{d}$ and $t>0$,

$$
\begin{aligned}
& \mathrm{E}\left(\left|u_{t}(x) u_{t}(y)\right|\right) \\
& \geq \mathrm{E}\left(u_{t}(x) u_{t}(y)\right) \\
& \quad \geq \eta^{2}+\int_{0}^{t} \mathrm{~d} s \int_{\mathbf{R}^{d}} \mathrm{~d} z \int_{\mathbf{R}^{d}} \mathrm{~d} z^{\prime} W_{s}\left(z, z^{\prime}\right) p_{t-s}(z-x) p_{t-s}\left(z^{\prime}-y\right) f\left(z-z^{\prime}\right),
\end{aligned}
$$

where

$$
W_{s}\left(z, z^{\prime}\right):=\mathrm{E}\left(\sigma\left(u_{s}(z)\right) \sigma\left(u_{s}\left(z^{\prime}\right)\right) \geq \mathrm{L}_{\sigma}^{2} \mathrm{E}\left(\left|u_{s}(z) u_{s}\left(z^{\prime}\right)\right|\right) .\right.
$$

Consider the following $\mathbf{R}_{+}$-valued functions on $\left(\mathbf{R}^{d}\right)^{2}$ :

$$
\begin{aligned}
H_{\beta}(a, b) & :=\int_{0}^{\infty} \mathrm{e}^{-\beta t} \mathrm{E}\left(\left|u_{t}(a) u_{t}(b)\right|\right) \mathrm{d} t \\
G_{\beta}(a, b) & :=\int_{0}^{\infty} \mathrm{e}^{-\beta t} p_{t}(a) p_{t}(b) \mathrm{d} t \\
F(a, b) & :=f(a-b) .
\end{aligned}
$$

Also consider the linear operator $\mathcal{A}_{\beta}$ defined as follows: For all nonnegative Borelmeasurable functions $h:\left(\mathbf{R}^{d}\right)^{2} \rightarrow \mathbf{R}_{+}$,

$$
\left(\mathcal{A}_{\beta} h\right)(x, y):=\left(F h * \tilde{G}_{\beta}\right)(x, y) .
$$

A line or two of computation shows that the preceding is simply a quick way to write the following:

$$
\begin{aligned}
& \left(\mathcal{A}_{\beta} h\right)(x, y) \\
& =\int_{\mathbf{R}^{d}} \mathrm{~d} a \int_{\mathbf{R}^{d}} \mathrm{~d} b F(a, b) h(a, b) G_{\beta}(x-a, y-b) \\
& =\int_{0}^{\infty} \mathrm{e}^{-\beta t} \mathrm{~d} t \int_{\mathbf{R}^{d}} \mathrm{~d} a \int_{\mathbf{R}^{d}} \mathrm{~d} b f(a-b) h(a, b) p_{t}(x-a) p_{t}(y-a) .
\end{aligned}
$$

With the preceding definitions under way, we can write equation (5.39) in shorthand as follows:

$$
H_{\beta}(x, y) \geq \frac{\eta^{2}}{\beta}+\mathrm{L}_{\sigma}^{2}\left(\mathcal{A}_{\beta} H_{\beta}\right)(x, y)
$$


Since $F \geq 0$ on $\left(\mathbf{R}^{d}\right)^{2}$, we can apply the preceding to find the following pointwise bounds:

$$
\begin{aligned}
H_{\beta} & \geq \frac{\eta^{2}}{\beta} \mathbf{1}+\mathrm{L}_{\sigma}^{2}\left(\mathcal{A}_{\beta}\left\{\frac{\eta^{2}}{\beta}+\mathrm{L}_{\sigma}^{2}\left(\mathcal{A}_{\beta} H_{\beta}\right)\right\}\right) \\
& =\frac{\eta^{2}}{\beta} \mathbf{1}+\mathrm{L}_{\sigma}^{2} \frac{\eta^{2}}{\beta} \mathcal{A}_{\beta} \mathbf{1}+\mathrm{L}_{\sigma}^{4} \mathcal{A}_{\beta}^{2} H_{\beta} \\
& \geq \frac{\eta^{2}}{\beta} \mathbf{1}+\mathrm{L}_{\sigma}^{2} \frac{\eta^{2}}{\beta} \mathcal{A}_{\beta} \mathbf{1}+\mathrm{L}_{\sigma}^{4} \mathcal{A}_{\beta}^{2}\left(\frac{\eta^{2}}{\beta}+\mathrm{L}_{\sigma}^{2} \mathcal{A}_{\beta} H_{\beta}\right) \\
& =\frac{\eta^{2}}{\beta} \mathbf{1}+\mathrm{L}_{\sigma}^{2} \frac{\eta^{2}}{\beta} \mathcal{A}_{\beta} \mathbf{1}+\mathrm{L}_{\sigma}^{4} \frac{\eta^{2}}{\beta} \mathcal{A}_{\beta}^{2} \mathbf{1}+\mathrm{L}_{\sigma}^{6} \mathcal{A}_{\beta}^{3} H_{\beta},
\end{aligned}
$$

where $\mathbf{1}(x, y):=1$ for all $x, y \in \mathbf{R}^{d}$. By applying induction we may arrive at the following simplified bound:

$$
H_{\beta} \geq \frac{\eta^{2}}{\beta} \cdot \sum_{\ell=0}^{k} \mathrm{~L}_{\sigma}^{2 \ell} \mathcal{A}_{\beta}^{\ell} \mathbf{1}+\mathrm{L}_{\sigma}^{2(k+1)} \mathcal{A}_{\beta}^{k+1} H_{\beta} \geq \frac{\eta^{2}}{\beta} \cdot \sum_{\ell=0}^{k} \mathrm{~L}_{\sigma}^{2 \ell} \mathcal{A}_{\beta}^{\ell} \mathbf{1} .
$$

Because the parameter $k \geq 0$ is arbitrary, it follows that

$$
H_{\beta} \geq \frac{\eta^{2}}{\beta} \cdot \sum_{\ell=0}^{\infty} \mathrm{L}_{\sigma}^{2 \ell} \mathcal{A}_{\beta}^{\ell} \mathbf{1}
$$

We analyse this infinite sum by using moment estimates that are motivated by Xiao's fractal analysis of fractional Brownian motion 94. In order to understand the structure of our estimates, we begin by inspecting only the first few terms.

The first term in the sum is identically 1 . The more interesting second term can be written as $\mathrm{L}_{\sigma}^{2}$ multipled by

$$
\begin{aligned}
\left(\mathcal{A}_{\beta} \mathbf{1}\right)(x, y) & =\left(F * \tilde{G}_{\beta}\right)(x, y) \\
& =\int_{0}^{\infty} \mathrm{e}^{-\beta t} \mathrm{~d} t \int_{\mathbf{R}^{d}} \mathrm{~d} a \int_{\mathbf{R}^{d}} \mathrm{~d} b f(a-b) p_{t}(a-x) p_{t}(b-y) \\
& \geq \frac{1}{(2 \pi)^{d}} \int_{0}^{\infty} \mathrm{e}^{-\beta t} \mathrm{~d} t \int_{\mathbf{R}^{d}} \mathrm{~d} \xi \hat{f}(\xi) \mathrm{e}^{-2 t \operatorname{Re} \Psi(\xi)} \mathrm{e}^{i \xi \cdot(x-y)},
\end{aligned}
$$

thanks to Proposition 3.3 . We change the order of the double integral $[\mathrm{d} t \mathrm{~d} \xi]$ to find that

$$
\left(\mathcal{A}_{\beta} \mathbf{1}\right)(x, y) \geq \frac{1}{(2 \pi)^{d}} \int_{\mathbf{R}^{d}} \frac{\mathrm{e}^{i \xi \cdot(x-y)} \hat{f}(\xi)}{\beta+2 \operatorname{Re} \Psi(\xi)} \mathrm{d} \xi
$$

[Theorem 1.2 and condition (1.1) together justify the use of Fubini's theorem.] 
In order to bound the third term in the infinite sum in (5.47), we need to estimate the following quantity:

$$
\begin{aligned}
\left(\mathcal{A}_{\beta}^{2} \mathbf{1}\right)(x, y) & =\left(F\left(\mathcal{A}_{\beta} \mathbf{1}\right) * \tilde{G}_{\beta}\right)(x, y) \\
& \geq \frac{1}{(2 \pi)^{d}} \int_{\mathbf{R}^{d}} \frac{\hat{f}(\xi) \mathrm{d} \xi}{\beta+2 \operatorname{Re} \Psi(\xi)} Z_{\xi}(x, y),
\end{aligned}
$$

where

$$
Z_{\xi}(x, y):=\int_{0}^{\infty} \mathrm{e}^{-\beta t} \mathrm{~d} t \int_{\mathbf{R}^{d}} \mathrm{~d} a \int_{\mathbf{R}^{d}} \mathrm{~d} b f(a-b) \mathrm{e}^{i \xi \cdot(a-b)} p_{t}(a-x) p_{t}(b-y) .
$$

[The same ideas that were applied to the second term can be applied here, in exactly the same manner, to produce this bound.] The Fourier transform of the function $a \mapsto \exp (i \xi \cdot a) p_{t}(a-x)$ is $\zeta \mapsto \exp (i(\xi+\zeta) \cdot x-t \Psi(\xi+\zeta))$. Therefore, Proposition 3.3 implies the following:

$$
\begin{aligned}
Z_{\xi}(x, y) & \geq \frac{1}{(2 \pi)^{d}} \int_{0}^{\infty} \mathrm{e}^{-\beta t} \mathrm{~d} t \int_{\mathbf{R}^{d}} \mathrm{~d} \zeta \hat{f}(\zeta) \mathrm{e}^{i(\xi+\zeta) \cdot(x-y)-2 t \operatorname{Re} \Psi(\xi+\zeta)} \\
& =\frac{1}{(2 \pi)^{d}} \int_{\mathbf{R}^{d}} \frac{\mathrm{e}^{i(\xi+\zeta) \cdot(x-y)} \hat{f}(\zeta)}{\beta+2 \operatorname{Re} \Psi(\xi+\zeta)} \mathrm{d} \zeta
\end{aligned}
$$

Therefore,

$$
\begin{aligned}
& \left(\mathcal{A}_{\beta}^{2} \mathbf{1}\right)(x, y) \\
& \geq \frac{1}{(2 \pi)^{2 d}} \int_{\mathbf{R}^{d}} \frac{\hat{f}\left(\xi_{1}\right) \mathrm{d} \xi_{1}}{\beta+2 \operatorname{Re} \Psi\left(\xi_{1}\right)} \int_{\mathbf{R}^{d}} \frac{\hat{f}\left(\xi_{2}\right) \mathrm{d} \xi_{2}}{\beta+2 \operatorname{Re} \Psi\left(\xi_{1}+\xi_{2}\right)} \mathrm{e}^{i\left(\xi_{1}+\xi_{2}\right) \cdot(x-y)} .
\end{aligned}
$$

Now, we can apply induction to deduce that we have the following estimate [used to analyse the $\ell$-th term in the infinite sum in (5.47)] in general: For all integers $\ell \geq 1$ and $x, y \in \mathbf{R}^{d}$,

$$
\begin{aligned}
& \left(\mathcal{A}_{\beta}^{\ell} \mathbf{1}\right)(x, y) \\
& \geq \frac{1}{(2 \pi)^{\ell d}} \int_{\mathbf{R}^{d}} \frac{\hat{f}\left(\xi_{1}\right) \mathrm{d} \xi_{1}}{\beta+2 \operatorname{Re} \Psi\left(\xi_{1}\right)} \int_{\mathbf{R}^{d}} \frac{\hat{f}\left(\xi_{2}\right) \mathrm{d} \xi_{2}}{\beta+2 \operatorname{Re} \Psi\left(\xi_{1}+\xi_{2}\right)} \\
& \quad \cdots \int_{\mathbf{R}^{d}} \frac{\hat{f}\left(\xi_{\ell}\right) \mathrm{d} \xi_{\ell}}{\beta+2 \operatorname{Re} \Psi\left(\xi_{1}+\cdots+\xi_{\ell}\right)} \mathrm{e}^{i \sum_{j=1}^{\ell} \xi_{j} \cdot(x-y) .}
\end{aligned}
$$

Although the preceding multiple integral is manifestly nonnegative, the integrand itself is complex valued. Fortunately, we wish to only understand the behavior of $\mathcal{F}_{\beta}^{\ell} \mathbf{1}$ on the diagonal of $\left(\mathbf{R}^{d}\right)^{2}$. In that case, the integrand is real and nonnegative. As such, we can estimate the integrand directly. In order to do so, let us set $y:=x$ in (5.54) and then plug the result in (5.47), to find that

$$
\begin{aligned}
& \inf _{x \in \mathbf{R}^{d}} \int_{0}^{\infty} \mathrm{e}^{-\beta t} \mathrm{E}\left(\left|u_{t}(x)\right|^{2}\right) \mathrm{d} t \\
& \geq \frac{\eta^{2}}{\beta} \cdot \sum_{\ell=0}^{\infty} \frac{\mathrm{L}_{\sigma}^{2 \ell}}{(2 \pi)^{\ell d}} \int_{\mathbf{R}^{d}} \mathrm{~d} \xi_{1} \cdots \int_{\mathbf{R}^{d}} \mathrm{~d} \xi_{\ell} \prod_{j=1}^{\ell} \frac{\hat{f}\left(\xi_{j}\right)}{\left(\beta+2 \operatorname{Re} \Psi\left(\xi_{1}+\cdots+\xi_{j}\right)\right)} .
\end{aligned}
$$


A change of variables yields the following:

$$
\begin{aligned}
\inf _{x \in \mathbf{R}^{d}} \int_{0}^{\infty} & \mathrm{e}^{-\beta t} \mathrm{E}\left(\left|u_{t}(x)\right|^{2}\right) \mathrm{d} t \\
& \geq \frac{\eta^{2}}{\beta} \cdot \sum_{\ell=0}^{\infty} \frac{\mathrm{L}_{\sigma}^{2 \ell}}{(2 \pi)^{\ell d}} \int_{\mathbf{R}^{d}} \mathrm{~d} z_{1} \cdots \int_{\mathbf{R}^{d}} \mathrm{~d} z_{d} \prod_{j=1}^{\ell} \frac{\hat{f}\left(z_{j}-z_{j-1}\right)}{\left(\beta+2 \operatorname{Re} \Psi\left(z_{j}\right)\right)},
\end{aligned}
$$

where $z_{0}:=0$.

The preceding holds even without Condition 1.7. But now we recall that Condition 1.7 is in place and use it to produce the announced lower bound on $\inf _{x \in \mathbf{R}^{d}} \bar{\gamma}_{x}(2)$.

Define

$$
\Sigma:=\left\{x:=\left(x_{1}, \ldots, x_{d}\right) \in \mathbf{R}^{d}: \operatorname{sign}\left(x_{1}\right)=\cdots=\operatorname{sign}\left(x_{d}\right)\right\} .
$$

Equivalently, $\Sigma:=\mathbf{R}_{+}^{d} \cup \mathbf{R}_{-}^{d}$. Because the terms under the product sign in (5.56) are all individually nonnegative, Condition 1.7 assures us that the following holds:

$$
\begin{aligned}
\inf _{x \in \mathbf{R}^{d}} \int_{0}^{\infty} \mathrm{e}^{-\beta t} \mathrm{E}\left(\left|u_{t}(x)\right|^{2}\right) \mathrm{d} t & \\
& \geq \frac{\eta^{2}}{\beta} \cdot \sum_{\ell=0}^{\infty} \frac{\mathrm{L}_{\sigma}^{2 \ell}}{(2 \pi)^{\ell d}} \int_{\Sigma} \mathrm{d} z_{1} \cdots \int_{\Sigma} \mathrm{d} z_{d} \prod_{j=1}^{\ell} \frac{\hat{f}\left(z_{j}-z_{j-1}\right)}{\left(\beta+2 \operatorname{Re} \Psi\left(z_{j}\right)\right)} .
\end{aligned}
$$

If $z_{1}, \ldots, z_{d} \in \Sigma$, then the absolute value of the $k$-th coordinate of $z_{j}-z_{j-1}$ is less than or equal to the absolute value of the $k$-th coordinate of $z_{j}$ for all $k=1, \ldots d$; therefore

$$
\hat{f}\left(z_{j}-z_{j-1}\right) \geq \hat{f}\left(z_{j}\right)
$$

thanks to Condition 1.7. Consequently,

$$
\begin{aligned}
\inf _{x \in \mathbf{R}^{d}} \int_{0}^{\infty} \mathrm{e}^{-\beta t} \mathrm{E}\left(\left|u_{t}(x)\right|^{2}\right) \mathrm{d} t & \geq \frac{\eta^{2}}{\beta} \cdot \sum_{\ell=0}^{\infty} \frac{\mathrm{L}_{\sigma}^{2 \ell}}{(2 \pi)^{\ell d}} \int_{\Sigma^{\ell}} \mathrm{d} z \prod_{j=1}^{\ell} \frac{\hat{f}\left(z_{j}\right)}{\left(\beta+2 \operatorname{Re} \Psi\left(z_{j}\right)\right)} \\
& =\frac{\eta^{2}}{\beta} \sum_{\ell=0}^{\infty}\left(\frac{\mathrm{L}_{\sigma}^{2}}{(2 \pi)^{d}} \int_{\Sigma} \frac{\hat{f}(z)}{(\beta+2 \operatorname{Re} \Psi(z))} \mathrm{d} z\right)^{\ell} .
\end{aligned}
$$

In particular, if there exists $\beta>0$ such that

$$
\frac{1}{(2 \pi)^{d}} \int_{\Sigma} \frac{\hat{f}(\xi) \mathrm{d} \xi}{\beta+2 \operatorname{Re} \Psi(\xi)} \geq \mathrm{L}_{\sigma}^{-2}
$$

then

$$
\int_{0}^{\infty} \mathrm{e}^{-\beta t} \mathrm{E}\left(\left|u_{t}(x)\right|^{2}\right) \mathrm{d} t=\infty \quad \text { for all } x \in \mathbf{R}^{d} .
$$

Thanks to symmetry considerations, Condition 1.7 has the following consequence:

$$
\int_{\Sigma} \frac{\hat{f}(\xi) \mathrm{d} \xi}{\beta+2 \operatorname{Re} \Psi(\xi)}=2^{-d+1} \int_{\mathbf{R}^{d}} \frac{\hat{f}(\xi) \mathrm{d} \xi}{\beta+2 \operatorname{Re} \Psi(\xi)}=2^{-d+1} \Upsilon(\beta) .
$$

This and (5.61) together imply that (5.62) holds whenever $\Upsilon(\beta) \geq 2^{d-1} / \mathrm{L}_{\sigma}^{2}$. Now we can apply a real-variable argument to prove that if (5.62) holds for some $\beta>0$, then

$$
\bar{\gamma}_{x}(2) \geq \beta \quad \text { for all } x \in \mathbf{R}^{d} .
$$

This and (5.38) together imply the theorem. 
Indeed, let us suppose to the contrary that (5.62) holds for our $\beta$, and yet $\inf _{x \in \mathbf{R}^{d}} \bar{\gamma}_{x}(2)<\beta$ for the very same $\beta$. It follows immediately that there exists $x \in \mathbf{R}^{d}, \delta \in(0, \beta)$, and $C \in(0, \infty)$ such that

$$
\mathrm{E}\left(\left|u_{t}(x)\right|^{2}\right) \leq C \mathrm{e}^{(\beta-\delta) t} \quad \text { for all } t>0 .
$$

Hence, (5.62) cannot hold in this case. This produces a contradiction and shows that (5.62) implies the theorem.

Proof of Theorem 1.11, We begin as we did with (5.39), but can no longer apply the inequality in (5.40). To circumvent that, note that for all $q_{0} \in(0, q)$ there exists $A:=A\left(q_{0}\right) \in(0, \infty)$ such that $\sigma(y) \geq q_{0}|y|$ as soon as $|y|>A$. We have assumed that $\mathrm{P}\left\{u_{s}(y)>0\right\}=1$ for all $s>0$ and $y \in \mathbf{R}^{d}$. Therefore,

$$
\begin{aligned}
W_{s}\left(z, z^{\prime}\right) \geq & q_{0}^{2} \mathrm{E}\left(u_{s}(z) u_{s}\left(z^{\prime}\right) ; u_{s}(z) \wedge u_{s}\left(z^{\prime}\right)>A\right) \\
\geq & q_{0}^{2} \mathrm{E}\left(u_{s}(z) u_{s}\left(z^{\prime}\right)\right)-q_{0}^{2} A^{2}-q_{0} A \mathrm{E}\left(u_{s}(z) ; u_{s}(z)>A\right) \\
& \quad-q_{0} A \mathrm{E}\left(u_{s}\left(z^{\prime}\right) ; u_{s}\left(z^{\prime}\right)>A\right) \\
\geq & q_{0}^{2} \mathrm{E}\left(u_{s}(z) u_{s}\left(z^{\prime}\right)\right)-q_{0}^{2} A^{2}-q_{0} A\left\{\mathrm{E}\left(u_{s}(z)\right)+\mathrm{E}\left(u_{s}\left(z^{\prime}\right)\right)\right\} .
\end{aligned}
$$

On the other hand, (5.1) guarantees that

$$
0 \leq \mathrm{E}\left(u_{t}(x)\right)=\left(P_{t} u_{0}\right)(x) \leq\left\|u_{0}\right\|_{L^{\infty}\left(\mathbf{R}^{d}\right)} .
$$

Consequently,

$$
W_{s}\left(z, z^{\prime}\right) \geq q_{0}^{2}\left\{\mathrm{E}\left(u_{s}(z) u_{s}\left(z^{\prime}\right)\right)-A_{*}\right\}
$$

where

$$
A_{*}:=\max \left(A^{2}, 2\left\|u_{0}\right\|_{L^{\infty}\left(\mathbf{R}^{d}\right)}\right) .
$$

Now we apply the recursion argument in the proof of Theorem 1.8 and find the following pointwise bounds:

$$
\begin{aligned}
H_{\beta} & \geq \frac{\eta^{2}}{\beta}+q_{0}^{2}\left\{\mathcal{A}_{\beta} H_{\beta}-A_{*} \mathcal{A}_{\beta} \mathbf{1}\right\} \\
& \geq \frac{\eta^{2}}{\beta}+q_{0}^{2}\left(\frac{\eta^{2}}{\beta}-A_{*}\right) \mathcal{A}_{\beta} \mathbf{1}+q_{0}^{4} \mathcal{A}_{\beta}^{2} H_{\beta}-q_{0}^{4} A_{*} \mathcal{A}_{\beta}^{2} \mathbf{1}
\end{aligned}
$$

$$
\geq \frac{\eta^{2}}{\beta}+\left(\frac{\eta^{2}}{\beta}-A_{*}\right) \sum_{\ell=1}^{N} q_{0}^{2 \ell} \mathcal{A}_{\beta}^{\ell} \mathbf{1}+q_{0}^{2(N+1)}\left(\mathcal{A}_{\beta}^{N+1} H_{\beta}-A_{*} \mathcal{A}_{\beta}^{N+1} \mathbf{1}\right),
$$

valid for every integer $N \geq 1$. We apply the following obvious inequalities: $\eta^{2} / \beta \geq$ $\eta^{2} / \beta-A_{*}$ to the first term on the right; and $H_{\beta} \geq \eta^{2} / \beta$ to the last bracketed term, to find that for all integers $N \geq 1$,

$$
H_{\beta} \geq\left(\frac{\eta^{2}}{\beta}-A_{*}\right) \sum_{\ell=0}^{N+1} q_{0}^{2 \ell} \mathcal{A}_{\beta}^{\ell} \mathbf{1}
$$


We can now let $N \uparrow \infty$ and apply the same estimate that we used to derive (5.60), and deduce the following bound:

$$
\begin{aligned}
\inf _{x \in \mathbf{R}^{d}} \int_{0}^{\infty} \mathrm{e}^{-\beta t} \mathrm{E}\left(\left|u_{t}(x)\right|^{2}\right) \mathrm{d} t & \\
& \geq\left(\frac{\eta^{2}}{\beta}-A_{*}\right) \cdot \sum_{\ell=0}^{\infty}\left(\frac{q_{0}^{2}}{(2 \pi)^{d}} \int_{\Sigma} \frac{\hat{f}(z)}{(\beta+2 \operatorname{Re} \Psi(z))} \mathrm{d} z\right)^{\ell} \\
& =\left(\frac{\eta^{2}}{\beta}-A_{*}\right) \cdot \sum_{\ell=0}^{\infty}\left(\frac{q_{0}^{2}}{2^{d-1}} \Upsilon(\beta)\right)^{\ell}
\end{aligned}
$$

Thanks to Theorem 1.2, the preceding implies the following bound:

$$
\inf _{x \in \mathbf{R}^{d}} \int_{0}^{\infty} \mathrm{e}^{-\beta t} \mathrm{E}\left(\left|u_{t}(x)\right|^{2}\right) \mathrm{d} t \geq\left(\frac{\eta^{2}}{\beta}-A_{*}\right) \cdot \sum_{\ell=0}^{\infty}\left(\frac{q_{0}^{2}}{2^{d-1}}\left(\bar{R}_{\beta} f\right)(0)\right)^{\ell} .
$$

Because $\left(\bar{R}_{0} f\right)(0)=\infty$, we can find a $\beta_{0}>0$ such that

$$
\left(\bar{R}_{\beta_{0}} f\right)(0) \geq \frac{2^{d-1}}{q_{0}^{2}} .
$$

For that choice of $\beta_{0}$, the preceding sum diverges. Therefore,

$$
\int_{0}^{\infty} \mathrm{e}^{-\beta_{0} t} \mathrm{E}\left(\left|u_{t}(x)\right|^{2}\right) \mathrm{d} t=\infty, \quad \text { provided that } \quad \eta>\sqrt{\beta_{0} A_{*}} .
$$

This and an elementary real-variable argument together prove the theorem; confer with the discussion that led to (5.65) for further details.

5.4. The massive and dissipative operators. David Nualart asked us about the effect of the drift coefficient $b$ in (1.1) on the intermittent behavior of the solution to the stochastic heat equation (1.1). At present, we have only an answer to this in a special, but physically-interesting, family of cases.

Indeed, let us consider the stochastic heat equation

$$
\frac{\partial}{\partial t} u_{t}(x)=\left(\mathcal{L} u_{t}\right)(x)+\frac{\lambda}{2} u_{t}(x)+\sigma\left(u_{t}(x)\right) \dot{F}_{t}(x),
$$

where $x \in \mathbf{R}^{d}, t>0, \lambda \in \mathbf{R}$, and $\dot{F}$ is as before. Moreover, $\sigma: \mathbf{R} \rightarrow \mathbf{R}$ is Lipschitz continuous, also as before. That is, (5.76) corresponds to the drift-free stochastic heat equation for the massive operator $\mathcal{L}^{(\lambda)}:=\mathcal{L}+(\lambda / 2) I$ when $\lambda>0$, the dissipative operator $\mathcal{L}^{(\lambda)}=\mathcal{L}-|\lambda / 2| I$ when $\lambda<0$, and the free operator $\mathcal{L}^{(0)}=\mathcal{L}$ when $\lambda=0$. Of course, Dalang's theory [30] - applied with $b(u):=\lambda u / 2$ guarantees us of the existence of an a.s.-unique mild solution to (5.76).

The operator $\mathcal{L}^{(\lambda)}$ is the generator of the semigroup $\left\{P_{t}^{(\lambda)}\right\}_{t \geq 0}$ defined by

$$
\left(P_{t}^{(\lambda)} \phi\right)(x):=\mathrm{e}^{\lambda t / 2}\left(P_{t} \phi\right)(x) .
$$

This can be seen immediately by a semiformal differential of $t \mapsto P_{t}^{(\lambda)}$ at $t=0$, and it is easy to make the argument rigorous as well. The corresponding "transition functions" are given by $p_{t}^{(\lambda)}(y-x):=\mathrm{e}^{\lambda t / 2} p_{t}(y-x)$. The domain $\operatorname{Dom}\left[\mathcal{L}^{(\lambda)}\right]$ of the definition of $\mathcal{L}^{(\lambda)}$ is the same as $\operatorname{Dom}[\mathcal{L}]$, and

$$
\mathcal{L}^{(\lambda)} \phi=\mathcal{L} \phi+\frac{\lambda}{2} \phi \quad \text { for all } \phi \in \operatorname{Dom}\left[\mathcal{L}^{(\lambda)}\right] .
$$


Let $\left\{P_{t}^{*(\lambda)}\right\}_{t \geq 0}$ denote the adjoint [or dual, in probabilistic terms] semigroup. That is, $\left(P_{t}^{*(\lambda)} \phi\right)(x):=\mathrm{e}^{\lambda t / 2}\left(P_{t}^{*} \phi\right)(x)$, with corresponding transition functions, $p_{t}^{*(\lambda)}(y-x):=\mathrm{e}^{\lambda t / 2} p_{t}(x-y)$. Finally there is also a corresponding replica semigroup, $\left(\bar{P}_{t}^{(\lambda)} \phi\right)(x):=\mathrm{e}^{\lambda t}\left(\bar{P}_{t} \phi\right)(x)$, whose resolvent is described by the following:

$$
\left(\bar{R}_{\alpha}^{(\lambda)} \phi\right)(x):=\int_{0}^{\infty} \mathrm{e}^{-(\alpha-\lambda) s}\left(\bar{P}_{s} \phi\right)(x) \mathrm{d} s \quad \text { for all } \alpha \geq \lambda .
$$

We might note that $\bar{R}_{\alpha}^{(\lambda)} f=\bar{R}_{\alpha-\lambda} f$ is merely a shift of the free replica resolvent of $f$. Therefore, the proof of Theorem 1.3 goes through unhindered, and after accounting for the mentioned shift, produces the following:

Theorem 5.6. Suppose $u_{0}: \mathbf{R}^{d} \rightarrow \mathbf{R}$ is bounded and measurable. Then, under Condition 1.1, the mild solution to (5.76) satisfies the following: For all integers $p \geq 2$ and $\lambda \in \mathbf{R}$,

$$
\bar{\gamma}_{*}(p) \leq \lambda+\left(\frac{p}{2}\right) \inf \left\{\alpha>0:\left(\bar{R}_{\alpha} f\right)(0)<\frac{1}{z_{p}^{2} \operatorname{Lip}_{\sigma}^{2}}\right\},
$$

where $z_{p}$ denotes the largest positive zero of the Hermite polynomial $\mathrm{He}_{p}$.

Recall the function $Q$ of Theorem 1.3 . Since

$$
Q(p, \beta) \geq \max \left(\frac{p \lambda}{2 \beta}, z_{p} \operatorname{Lip}_{\sigma} \sqrt{\left(\bar{R}_{2 \beta / p} f\right)(0)}\right)
$$

a few lines of arithmetic show that Theorem 5.6 provides us with a better upper bound than Theorem 1.3 for the top Liapounov $L^{p}$-exponent of the mild solution to (1.1). Next, we produce instances where the solution is intermittent.

First of all, note that according to (5.80),

$$
\bar{\gamma}_{*}(2) \leq \lambda+\inf \left\{\alpha>0:\left(\bar{R}_{\alpha} f\right)(0)<\frac{1}{\operatorname{Lip}_{\sigma}^{2}}\right\}
$$

because $z_{2}=2$. We apply similar "shifting arguments", together with Theorem 1.8 , to deduce that the following offers a converse, under some symmetry and regularity conditions. We note, in advance, that when $d=1$, the preceding estimate and the following essentially match up.

Theorem 5.7. Suppose that both Conditions 1.1 and 1.7 hold, $\eta:=\inf _{x \in \mathbf{R}^{d}} u_{0}(x)$ $>0$, and there exists $\mathrm{L}_{\sigma} \in(0, \infty)$ such that $\sigma(z) \geq \mathrm{L}_{\sigma}|z|$ for all $z \in \mathbf{R}$. Then,

$$
\inf _{x \in \mathbf{R}^{d}} \bar{\gamma}_{x}(2) \geq \lambda+\sup \left\{\alpha>0:\left(\bar{R}_{\alpha} f\right)(0) \geq \frac{2^{d-1}}{\mathrm{~L}_{\sigma}^{2}}\right\},
$$

where $\sup \varnothing:=0$.

We end this section with an example mentioned in the Introduction.

Example 5.8. Consider the case where $\mathcal{L}=-(-\Delta)^{q / 2}$ is a power of the Laplacian. In that case, $\mathcal{L}$ is the generator of an isotropic stable process of index $q$, and $q \in(0,2]$ necessarily. Also consider the case where $f(x)=\|x\|^{-d+b}$ is a Riesz kernel, where $b \in(0, d)$. Then [see (3.15)],

$$
\hat{f}(\xi)=\frac{C_{d, b}}{\|\xi\|^{b}}, \quad \text { where } \quad C_{d, b}:=\frac{\pi^{d / 2} 2^{b} \Gamma(b / 2)}{\Gamma((d-b) / 2)} .
$$


Therefore, Theorem 1.2 and direct computation together imply that

$$
\begin{aligned}
\left(\bar{R}_{\alpha} f\right)(0) & =\Upsilon(\alpha)=\frac{C_{d, b}}{(2 \pi)^{d}} \cdot \int_{\mathbf{R}^{d}} \frac{\|\xi\|^{-b}}{\alpha+2\|\xi\|^{q}} \mathrm{~d} \xi \\
& =\frac{C_{d, b} \alpha^{-1+(d-b) / q}}{(2 \pi)^{d} \cdot 2^{(d-b) / q}} \cdot \int_{\mathbf{R}^{d}} \frac{\mathrm{d} z}{\|z\|^{b}+\|z\|^{q+b}}
\end{aligned}
$$

In other words,

$$
\left(\bar{R}_{\alpha} f\right)(0)=\frac{\mathfrak{A}_{d, q, b}}{\alpha^{1-\nu}}
$$

where

$$
\nu:=\frac{d-b}{q} \quad \text { and } \quad \mathfrak{A}_{d, q, b}:=\frac{C_{d, b}}{(2 \pi)^{d} \cdot 2^{\nu}} \cdot \int_{\mathbf{R}^{d}} \frac{\mathrm{d} z}{\|z\|^{b}+\|z\|^{q+b}} .
$$

Since $b \in(0, d), \mathfrak{A}_{d, q, b}$ - and hence $\left(\bar{R}_{\alpha} f\right)(0)$-is finite if and only if $q+b>d$, this is the sufficient condition for the existence of a unique mild solution to the resulting stochastic PDE. Not surprisingly, this " $q+b>d$ " condition is the necessary and sufficient condition for the existence of a solution to the linear equation [Theorem 4.5. Moreover, when $q+b>d$, we can apply Theorems 5.6 and 5.7 to find that for all $x \in \mathbf{R}^{d}$,

$$
\lambda+\left(\frac{\mathfrak{A}_{d, q, b} \mathrm{~L}_{\sigma}^{2}}{2^{d-1}}\right)^{1 /(1-\nu)} \leq \bar{\gamma}_{x}(2) \leq \lambda+\left(\mathfrak{A}_{d, q, b} \operatorname{Lip}_{\sigma}^{2}\right)^{1 /(1-\nu)}
$$

In particular, consider the massive/dissipative "parabolic Anderson model",

$$
\frac{\partial}{\partial t} u_{t}(x)=-\left((\Delta)^{q / 2} u_{t}\right)(x)+\frac{\lambda}{2} u_{t}(x)+\kappa u_{t}(x) \dot{F}_{t}(x)
$$

where $u_{0}: \mathbf{R} \rightarrow \mathbf{R}$ is measurable and bounded uniformly away from zero and infinity, and $\kappa \neq 0$. The preceding discussion shows that the parabolic Anderson model has a solution if $q+b>d$. When $q+b>d$, we obtain the following bounds for the upper $L^{2}$-Liapounov exponent of the solution: For all $x \in \mathbf{R}^{d}$,

$$
\lambda+\left(\frac{\mathfrak{A}_{d, q, b} \kappa^{2}}{2^{d-1}}\right)^{1 /(1-\nu)} \leq \bar{\gamma}_{x}(2) \leq \lambda+\left(\mathfrak{A}_{d, q, b} \kappa^{2}\right)^{1 /(1-\nu)} .
$$

Theorem 1.3 also shows that $\bar{\gamma}_{*}(p)<\infty$ for all $p \geq 2$. Therefore, we have no weak intermittency if $\lambda \leq-\left(\mathfrak{A}_{d, q, b} \kappa^{2}\right)^{1 /(1-\nu)}$, whereas there is weak intermittency if $\lambda>-\left(\mathfrak{A}_{d, q, b} \kappa^{2} /\left(2^{d-1}\right)\right)^{1 /(1-\nu)}$. Our condition is sharp when, and only when, $d=1$. In that one-dimensional case, we have a solution if and only if $q+b>1$, and if this inequality holds, then

$$
\mathfrak{A}_{1, q, b}=\frac{C_{1, b}}{2^{\nu} \pi} \cdot \int_{0}^{\infty} \frac{\mathrm{d} z}{z^{b}+z^{b+q}}=\frac{C_{1, b}}{2^{\nu} \pi q} \cdot \mathrm{B}\left(\frac{1-b}{q}, 1-\frac{1-b}{q}\right),
$$

where $\mathrm{B}(x, y):=\Gamma(x) \Gamma(y) / \Gamma(x+y)$ denotes the beta function. Thanks to (5.84), this implies that

$$
\mathfrak{A}_{1, q, b}=\frac{2^{b-\nu} \Gamma(b / 2)}{\pi^{1 / 2} q \Gamma((1-b) / 2)} \mathrm{B}\left(\frac{1-b}{q}, 1-\frac{1-b}{q}\right),
$$


and we find that weak intermittency holds if and only if

$$
\lambda>-\left(\mathfrak{A}_{1, q, b} \kappa^{2}\right)^{1 /(1-\nu)} .
$$

Another simple though tedious computation shows that this example [applied with $q:=2$ ] includes the material that led to (1.22).

\section{ACKNOWLEDGEMENTS}

The authors learned about the stochastic heat equation with spatially-correlated forcing from Michael Cranston, David Nualart asked about the interplay between drift and intermittence, Steven Zelditch told the authors about [96], and Daniel Conus made detailed remarks and thoughtful suggestions on this topic. We thank them all.

\section{REFERENCES}

1. S. Assing and R. Manthey, The behavior of solutions of stochastic differential inequalities, Probab. Theory Related Fields 103 (1995), no. 4, 493-514. MR.1360202 (97c:60147)

2. R. F. Bass and M. Cranston, The Malliavin calculus for pure jump processes and applications to local time, Ann. Probab. 14 (1986), no. 2, 490-532. MR.832021 (88b:60113)

3. Richard Bass, $L_{p}$ inequalities for functionals of Brownian motion, Séminaire de Probabilités, XXI, Lecture Notes in Math., vol. 1247, Springer, Berlin, 1987, pp. 206-217. MR941984 (89h:60072)

4. L. Bertini, N. Cancrini, and G. Jona-Lasinio, The stochastic Burgers equation, Comm. Math. Phys. 165 (1994), no. 2, 211-232. MR1301846 (96d:60092)

5. Lorenzo Bertini and Nicoletta Cancrini, The stochastic heat equation: Feynman-Kac formula and intermittence, J. Statist. Phys. 78 (1995), no. 5-6, 1377-1401. MR.1316109 (95j:60093)

6. The two-dimensional stochastic heat equation: Renormalizing a multiplicative noise, J. Phys. A 31 (1998), no. 2, 615-622. MR1629198(99c:82051)

7. Lorenzo Bertini and Giambattista Giacomin, Stochastic Burgers and KPZ equations from particle systems, Comm. Math. Phys. 183 (1997), no. 3, 571-607. MR1462228 (99e:60212)

8. On the long-time behavior of the stochastic heat equation, Probab. Theory Related Fields 114 (1999), no. 3, 279-289. MR1705123 (2000k:60123)

9. Jean Bertoin, Lévy Processes, Cambridge Tracts in Mathematics, vol. 121, Cambridge University Press, Cambridge, 1996. MR,1406564 (98e:60117)

10. Subordinators: Examples and Applications, Lectures on Probability Theory and Statistics (Saint-Flour, 1997), Lecture Notes in Math., vol. 1717, Springer, Berlin, 1999, pp. 1-91. MR.1746300 (2002a:60001)

11. J. R. Blum and Murray Rosenblatt, On the structure of infinitely divisible distributions, Pacific J. Math. 9 (1959), 1-7. MR0105729 (21:4465)

12. R. M. Blumenthal and R. K. Getoor, Sample functions of stochastic processes with stationary independent increments, J. Math. Mech. 10 (1961), 493-516. MR0123362 (23:A689)

13. _ Markov Processes and Potential Theory, Pure and Applied Mathematics, Vol. 29, Academic Press, New York, 1968. MR0264757 (41:9348)

14. _ Dual processes and potential theory, Proc. Twelfth Biennial Sem. Canad. Math. Congr. on Time Series and Stochastic Processes; Convexity and Combinatorics (Vancouver, B.C., 1969), Canad. Math. Congr., Montreal, Que., 1970, pp. 137-156. MR0273685(42:8562)

15. Salomon Bochner, Harmonic Analysis and the Theory of Probability, University of California Press, Berkeley and Los Angeles, 1955. MR0072370(17:273d)

16. Eric Carlen and Paul Krée, $L^{p}$ estimates on iterated stochastic integrals, Ann. Probab. 19 (1991), no. 1, 354-368. MR 1085341 (92e:60085)

17. R. A. Carmona and S. A. Molchanov, Stationary parabolic Anderson model and intermittency, Probab. Theory Related Fields 102 (1995), no. 4, 433-453. MR.1346261 (96m:60142)

18. Rene Carmona, Leonid Koralov, and Stanislav Molchanov, Asymptotics for the almost sure Lyapunov exponent for the solution of the parabolic Anderson problem, Random Oper. Stochastic Equations 9 (2001), no. 1, 77-86. MR1910468 (2003g:60104) 
19. René A. Carmona and S. A. Molchanov, Parabolic Anderson Problem and Intermittency, Mem. Amer. Math. Soc. 108 (1994), no. 518, viii+125. MR.1185878 (94h:35080)

20. René A. Carmona and Frederi G. Viens, Almost-sure exponential behavior of a stochastic Anderson model with continuous space parameter, Stochastics Stochastics Rep. 62 (1998), no. 3-4, 251-273. MR.1615092 (99c:60126)

21. Gustave Choquet and Jacques Deny, Sur l'équation de convolution $\mu=\mu * \sigma$, C. R. Acad. Sci. Paris 250 (1960), 799-801. MR0119041 (22:9808)

22. Francis Comets, Tokuzo Shiga, and Nobuo Yoshida, Directed polymers in a random environment: Path localization and strong disorder, Bernoulli 9 (2003), no. 4, 705-723. MR1996276 (2004f:60210)

23. Probabilistic analysis of directed polymers in a random environment: A review, Stochastic analysis on large scale interacting systems, Adv. Stud. Pure Math., vol. 39, Math. Soc. Japan, Tokyo, 2004, pp. 115-142. MR2073332 (2005d:82050)

24. Francis Comets and Nobuo Yoshida, Brownian directed polymers in random environment, Comm. Math. Phys. 254 (2005), no. 2, 257-287. MR2117626 (2005m:60242)

25. Daniel Conus and Robert C. Dalang, The non-linear stochastic wave equation in high dimensions, Electron. J. Probab. 13 (2008), no. 22, 629-670. MR2399293 (2009c:60170)

26. M. Cranston and S. Molchanov, On phase transitions and limit theorems for homopolymers, Probability and Mathematical Physics, CRM Proc. Lecture Notes, vol. 42, Amer. Math. Soc., Providence, RI, 2007, pp. 97-112. MR2352264 (2009a:60119)

27. _ Q Quenched to annealed transition in the parabolic Anderson problem, Probab. Theory Related Fields 138 (2007), no. 1-2, 177-193. MR2288068(2008h:60066)

28. M. Cranston, T. S. Mountford, and T. Shiga, Lyapunov exponents for the parabolic Anderson model, Acta Math. Univ. Comenian. (N.S.) 71 (2002), no. 2, 163-188. MR.1980378 (2004d:60162)

29. Lyapunov exponent for the parabolic Anderson model with Lévy noise, Probab. Theory Related Fields 132 (2005), no. 3, 321-355. MR2197105(2007h:60053)

30. Robert C. Dalang, Extending the martingale measure stochastic integral with applications to spatially homogeneous s.p.d.e.'s, Electron. J. Probab. 4 (1999), no. 6, 29 pp. (electronic). MR1684157 (2000b:60132)

31. Robert C. Dalang and N. E. Frangos, The stochastic wave equation in two spatial dimensions, Ann. Probab. 26 (1998), no. 1, 187-212. MR1617046 (99c:60127)

32. Robert C. Dalang and Olivier Lévêque, Second-order hyperbolic S.P.D.E. 's driven by boundary noises, Seminar on Stochastic Analysis, Random Fields and Applications IV, Progr. Probab., vol. 58, Birkhäuser, Basel, 2004, pp. 83-93. MR2096282

33. Second-order linear hyperbolic SPDEs driven by isotropic Gaussian noise on a sphere, Ann. Probab. 32 (2004), no. 1B, 1068-1099. MR2044674 (2005h:60182)

34. - Second-order hyperbolic S.P.D.E.'s driven by homogeneous Gaussian noise on a hyperplane, Trans. Amer. Math. Soc. 358 (2006), no. 5, 2123-2159 (electronic). MR.2197451 (2006k:60112)

35. Robert C. Dalang and Carl Mueller, Some non-linear S.P.D.E.'s that are second order in time, Electron. J. Probab. 8 (2003), no. 1, 21 pp. (electronic). MR1961163 (2004a:60118)

36. Robert C. Dalang, Carl Mueller, and Roger Tribe, A Feynman-Kac-type formula for the deterministic and stochastic wave equations and other P.D.E.'s, Trans. Amer. Math. Soc. 360 (2008), no. 9, 4681-4703. MR2403701 (2009e:60146)

37. Robert C. Dalang and Marta Sanz-Solé, Hölder-Sobolev regularity of the solution to the stochastic wave equation in dimension three, Mem. Amer. Math. Soc. 199 (2009), no. 931, vi+70. MR2512755

38. Burgess Davis, On the $L^{p}$ norms of stochastic integrals and other martingales, Duke Math. J. 43 (1976), no. 4, 697-704. MR0418219(54:6260)

39. D. A. Dawson, I. Iscoe, and E. A. Perkins, Super-Brownian motion: Path properties and hitting probabilities, Probab. Theory Related Fields 83 (1989), no. 1-2, 135-205. MR.1012498 (90k:60073)

40. C. Donati-Martin and É. Pardoux, White noise driven SPDEs with reflection, Probab. Theory Related Fields 95 (1993), no. 1, 1-24. MR.1207304 (94f:60083)

41. E. B. Dynkin, Polynomials of the occupation field and related random fields, J. Funct. Anal. 58 (1984), no. 1, 20-52. MR756768 (86h:60085b) 
42. Nathalie Eisenbaum, Mohammud Foondun, and Davar Khoshnevisan, Dynkin's isomorphism and the stochastic heat equation, Potential Analysis 3 (2011), no. 3, 243-260. MR 2782972

43. M. Fisz and V. S. Varadarajan, A condition for absolute continuity of infinitely divisible distribution functions, Z. Wahrscheinlichkeitstheorie und Verw. Gebiete 1 (1962/1963), 335339. MR0149521 (26:7007)

44. Ionut Florescu and Frederi Viens, Sharp estimation of the almost-sure Lyapunov exponent for the Anderson model in continuous space, Probab. Theory Related Fields 135 (2006), no. 4, 603-644. MR2240702(2008g:60189)

45. Mohammud Foondun and Davar Khoshnevisan, Intermittence and nonlinear parabolic stochastic partial differential equations, Electron. J. Probab. 14 (2009), no. 21, 548-568. MR.2480553

46. Mohammud Foondun, Davar Khoshnevisan, and Eulalia Nualart, A local time correspondence for stochastic partial differential equations, Trans. Amer. Math. Soc. 363 (2011), no. 5, 24812515. MR2763724

47. Masatoshi Fukushima, Yōichi Ōshima, and Masayoshi Takeda, Dirichlet Forms and Symmetric Markov Processes, de Gruyter Studies in Mathematics, vol. 19, Walter de Gruyter \& Co., Berlin, 1994. MR1303354 (96f:60126)

48. J. Gärtner and F. den Hollander, Intermittency in a catalytic random medium, Ann. Probab. 34 (2006), no. 6, 2219-2287. MR2294981(2008e:60200)

49. Jürgen Gärtner and Wolfgang König, The parabolic Anderson model, Interacting Stochastic Systems, Springer, Berlin, 2005, pp. 153-179. MR2118574(2005k:82042)

50. I. M. Gel'fand and N. Ya. Vilenkin, Generalized Functions. Vol. 4, Academic Press [Harcourt Brace Jovanovich Publishers], New York, 1964 [1977], Applications of harmonic analysis, Translated from the Russian by Amiel Feinstein. MR 0435834 (55:8786d)

51. I. V. Girsanov, Strong Feller processes. I. General properties, Teor. Verojatnost. i Primenen. 5 (1960), 7-28. MR0137151 (25:607)

52. Philip Hartman and Aurel Wintner, On the infinitesimal generators of integral convolutions, Amer. J. Math. 64 (1942), 273-298. MR0006635 (4:18a)

53. U. G. Haussmann and É. Pardoux, Stochastic variational inequalities of parabolic type, Appl. Math. Optim. 20 (1989), no. 2, 163-192. MR998402 (90k:60119)

54. John Hawkes, Potential theory of Lévy processes, Proc. London Math. Soc. (3) 38 (1979), no. 2, 335-352. MR.531166 (80g:60077)

55. _ Transition and resolvent densities for Lévy processes, Unpublished manuscript (1984).

56. Edwin Hewitt and Leonard J. Savage, Symmetric measures on Cartesian products, Trans. Amer. Math. Soc. 80 (1955), 470-501. MR0076206 (17:863g)

57. Yaozhong Hu and David Nualart, Stochastic heat equation driven by fractional noise and local time, Probab. Theory Related Fields 143 (2009), no. 1-2, 285-328. MR.2449130

58. N. Jacob, Pseudo Differential Operators and Markov Processes. Vol. III, Imperial College Press, London, 2005, Markov processes and applications. MR2158336 (2006i:60001)

59. Mehran Kardar, Replica Bethe ansatz studies of two-dimensional interfaces with quenched random impurities, Nuclear Phys. B 290 (1987), no. 4, 582-602. MR922846 (89f:82058)

60. Mehran Kardar, Giorgio Parisi, and Yi-Cheng Zhang, Dynamical scaling of growing surfaces, Phys. Rev. Lett. 56 (1986), no. 9, 889-892.

61. Davar Khoshnevisan and Yimin Xiao, Harmonic analysis of additive Lévy processes, Probab. Theory Related Fields 145 (2009), 459-515. MR2529437(2010i:60157)

62. Wolfgang König, Hubert Lacoin, Peter Mörters, and Nadia Sidorova, A two cities theorem for the parabolic Anderson model, Ann. Probab. 37 (2009), no. 1, 347-392. MR2489168

63. Peter Kotelenez, Comparison methods for a class of function valued stochastic partial differential equations, Probab. Theory Related Fields 93 (1992), no. 1, 1-19. MR1172936 (93i:60116)

64. H. Krug and H. Spohn, Kinetic roughening of growing surfaces, Solids Far From Equilibrium: Growth, Morphology, and Defects (Claude Godréche, ed.), Cambridge University Press, Cambridge, 1991, pp. 412-525.

65. Andreas E. Kyprianou, Introductory Lectures on Fluctuations of Lévy Processes with Applications, Universitext, Springer-Verlag, Berlin, 2006. MR2250061 (2008a:60003)

66. Paul Lévy, Théorie de l'Addition des Variables Aléatoires, Gauthier-Villars, 1937.

67. Elliott H. Lieb and Werner Liniger, Exact analysis of an interacting Bose gas. I. The general solution and the ground state, Phys. Rev. (2) 130 (1963), 1605-1616. MR0156630 (27:6551) 
68. Alessandra Lunardi, Analytic Semigroups and Optimal Regularity in Parabolic Problems, Progress in Nonlinear Differential Equations and their Applications, 16, Birkhäuser Verlag, Basel, 1995. MR1329547 (96e:47039)

69. Ralf Manthey, Existence and uniqueness of a solution of a reaction-diffusion equation with polynomial nonlinearity and white noise disturbance, Math. Nachr. 125 (1986), 121-133. MR847354 (87j:60092)

70. Ralf Manthey and Christel Stiewe, On Volterra's population equation with diffusion and noise, Stochastic Processes and Related Topics (Georgenthal, 1990), Math. Res., vol. 61, AkademieVerlag, Berlin, 1991, pp. 89-92. MR.1127884

71. Existence and uniqueness of solutions to Volterra's population equation with diffusion and noise, Stochastics Stochastics Rep. 41 (1992), no. 3, 135-161. MR1275580 (95b:60072)

72. Michael B. Marcus and Jay Rosen, Markov Processes, Gaussian Processes, and Local Times, Cambridge Studies in Advanced Mathematics, vol. 100, Cambridge University Press, Cambridge, 2006. MR2250510 (2008b:60001)

73. Pertti Mattila, Geometry of Sets and Measures in Euclidean Spaces, Cambridge Studies in Advanced Mathematics, vol. 44, Cambridge University Press, Cambridge, 1995, Fractals and rectifiability. MR.1333890(96h:28006)

74. Henry P. McKean, Stochastic Integrals, AMS Chelsea Publishing, Providence, RI, 2005. Reprint of the 1969 edition, with errata. MR2169626 (2006d:60003)

75. Ernesto Medina, Terence Hwa, Mehran Kardar, and Yi-Chen Zhang, Burgers equation with correlated noise: Renormalization-group analysis and applications to directed polymers and interface growth, Phys. Rev. A 39 (1989), no. 6, 3053-3075. MR988871 (89m:82027)

76. P. W. Millar, Radial processes, Ann. Probability 1 (1973), 613-626. MR0353464 (50:5947)

77. Stanislav A. Molchanov, Ideas in the Theory of Random Media, Acta Appl. Math. 22 (1991), no. 2-3, 139-282. MR:1111743 (92m:82067)

78. Carl Mueller, On the support of solutions to the heat equation with noise, Stochastics Stochastics Rep. 37 (1991), no. 4, 225-245. MR1149348 (93e:60122)

79. Leonid Mytnik and Edwin Perkins, Regularity and irregularity of $(1+\beta)$-stable superBrownian motion, Ann. Probab. 31 (2003), no. 3, 1413-1440. MR1989438 (2004b:60130)

80. Ivan Nourdin and Thomas Simon, On the absolute continuity of Lévy processes with drift, Ann. Probab. 34 (2006), no. 3, 1035-1051. MR.2243878 (2007j:60073)

81. D. Nualart and É. Pardoux, White noise driven quasilinear SPDEs with reflection, Probab. Theory Related Fields 93 (1992), no. 1, 77-89. MR1172940 (93h:60093)

82. R. E. A. C. Paley and A. Zygmund, A note on analytic functions in the unit circle, Proc. Camb. Phil. Soc. 28 (1932), no. [Issue] 03, 266-272.

83. Szymon Peszat and Jerzy Zabczyk, Nonlinear stochastic wave and heat equations, Probab. Theory Related Fields 116 (2000), no. 3, 421-443. MR.1749283 (2001f:60071)

84. Daniel Revuz and Marc Yor, Continuous Martingales and Brownian Motion, Grundlehren der Mathematischen Wissenschaften [Fundamental Principles of Mathematical Sciences], vol. 293, Springer-Verlag, Berlin, 1991. MR1083357 (92d:60053)

85. Ken-iti Sato, Lévy Processes and Infinitely Divisible Distributions, Cambridge Studies in Advanced Mathematics, vol. 68, Cambridge University Press, Cambridge, 1999. Translated from the 1990 Japanese original, revised by the author. MR 1739520 (2003b:60064)

86. Tokuzo Shiga, Two contrasting properties of solutions for one-dimensional stochastic partial differential equations, Canad. J. Math. 46 (1994), no. 2, 415-437. MR1271224(95h:60099)

87. L Exponential decay rate of survival probability in a disastrous random environment, Probab. Theory Related Fields 108 (1997), no. 3, 417-439. MR1465166 (98f:60212)

88. Howard G. Tucker, Absolute continuity of infinitely divisible distributions, Pacific J. Math. 12 (1962), 1125-1129. MR0146868 (26:4387)

89. - On continuous singular infinitely divisible distribution functions, Ann. Math. Statist. 35 (1964), 330-335. MR0161362 (28:4569)

90. _ On a necessary and sufficient condition that an infinitely divisible distribution be absolutely continuous, Trans. Amer. Math. Soc. 118 (1965), 316-330. MR0182061(31:6285)

91. Remco van der Hofstad, Wolfgang König, and Peter Mörters, The universality classes in the parabolic Anderson model, Comm. Math. Phys. 267 (2006), no. 2, 307-353. MR2249772 (2007g:82029) 
92. John B. Walsh, An Introduction to Stochastic Partial Differential Equations, École d'été de Probabilités de Saint-Flour, XIV-1984, Lecture Notes in Math., vol. 1180, Springer, Berlin, 1986, pp. 265-439. MR876085 (88a:60114)

93. Wojbor A. Woyczyński, Burgers-KPZ Turbulence, Lecture Notes in Mathematics, vol. 1700, Springer-Verlag, Berlin, 1998, Göttingen lectures. MR.1732301 (2000j:60077)

94. Yimin Xiao, Packing dimension of the image of fractional Brownian motion, Statist. Probab. Lett. 33 (1997), no. 4, 379-387. MR1458008 (98g:60080)

95. J. Zabczyk, Sur la théorie semi-classique du potentiel pour les processus à accroissements indépendants, Studia Math. 35 (1970), 227-247. MR0267643(42:2545)

96. Ya. B. Zeldovich, A. A. Ruzmaikin, and D. D. Sokoloff, The Almighty Chance, World Scientific Lecture Notes in Physics, vol. 20, World Scientific, Singapore, 1990. MR1141627 (93b:82001)

School of Mathematics, Loughborough University, Leicestershire, Le11 3TU United KINGDOM

E-mail address: m.i.foondun@lboro.ac.uk

Department of Mathematics, University of Utah, 155 South 1400 East JWB 233, Salt LAKe City, UTAh 84112-0090

E-mail address: davar@math.utah.edu 